The copyright of this thesis vests in the author. No quotation from it or information derived from it is to be published without full acknowledgement of the source. The thesis is to be used for private study or noncommercial research purposes only.

Published by the University of Cape Town (UCT) in terms of the non-exclusive license granted to UCT by the author. 


\title{
CHARACTERISING THE POROSITY OF MULTI-COMPONENT MIXTURES IN ROTARY MILLS
}

Kateula Sichalwe

\author{
Thesis submitted for the Degree of \\ Master of Science in Engineering
}

Department of Chemical Engineering

University of Cape Town

February 2011 


\section{Declaration}

I hereby grant the University free license to reproduce the above thesis in whole or in part, for the purpose of research.

I also declare that:

a) I know the meaning of plagiarism and declare that all the work in the document, save for that which is properly acknowledged, is my own;

a) except as stated below, neither the substance or any part of the thesis has been submitted in the past, or is being, or is to be submitted for a degree in the University or any other University;

b) I am now presenting the thesis for examination for the Degree of Masters of Science in Engineering.

Kateula Sichalwe. 


\section{Acknowledgements}

I would like to acknowledge the following:

- Dr Indresan Govender for his unwavering supervision and patience, without which this would not be a success;

- Dr Aubrey N Mainza for helping me to start this work and for his supervision;

- My parents for love, guidance and motivation;

- My siblings for checking on me whenever I went missing in my library;

- My wife Mulemba for her love and support all the way;

- Anglo Platinum for financial assistance;

- CERECAM for office space and wonderful company;

- $\quad$ CMR and PEPT-UCT for being great research teams;

- God Almighty for all things! 
To my daughter

Lukundo Nachalwe 


\section{Synopsis}

Porosity, or fractional void volume, is a simple but powerful quantity used in describing porous media. Characterising this very important parameter is vital to understanding key processes that occur in porous media, such as fluid transport. This is because porosity is strongly related to the permeability of porous media. In tumbling mills, for example, slurry transport is poorly understood, mainly due to inadequate characterisation of porosity within the mill charge. In particular, most models of slurry transport in tumbling mills assume a uniform charge porosity equal to 0.4 - the porosity of a stationary loosely packed bed. These models, which are mostly empirical, are therefore very difficult to extrapolate beyond the window of experimental conditions under which they were designed.

Studies of porosity in rotating drums - similar to tumbling mills or rotary mills in generalshow that the porosity of the rotating bed within the drum exhibits a spatial distribution. This distribution has been shown to vary with mill operating parameters, such as mill speed and filling fraction. However, these studies consider very simple systems, mostly based on monosized and binary charge, and drums without lifters. Thus, due to the dominance of porosity in mechanistic slurry transport modelling, improved understanding of the spatial distribution of porosity is required. This would result in improved efficiency and huge energy savings; especially considering the effect of slurry transport efficiency on mill performance.

The aim of this study has been to improve knowledge of porosity in rotary mills and how this varies with key operating parameters. However, it is difficult to perform direct measurements in such aggressive systems. Therefore, non-invasive methods were sought after. In this work, the positron emission particle tracking (PEPT) method was adopted as a measurement tool for particle dynamics. The system studied is a $300 \mathrm{~mm}$ laboratory scale mill having particles of different sizes. Due to the limitation of PEPT to tracking only one radioactively labelled tracer, effectively, one major challenge has been how to study multi-components mixtures. In fact, to the best of my knowledge, there is no method for computing the porosity of such mixtures using PEPT. Accordingly, extending the methods used for monosized systems to include multi-component systems forms a large part of this thesis.

To represent the entire system (all size components) a particle representing each component was tracked, in turn. The system was assumed to be ergodic. Ergodicity implies that the 
fraction of total run time a tracer spends in a given region is proportional to the packing density of that region. Furthermore, assuming coexistence of size components, a single particle's trajectory data could be used to estimate the volume of solids its size component contributes to the given region. Consequently, the porosity of the entire system was modelled as a linear combination of the solid volume contributions for each component. Finally, the correlation of spatial distribution of porosity with operating parameters was investigated. 


\section{Table of Contents}

Declaration...

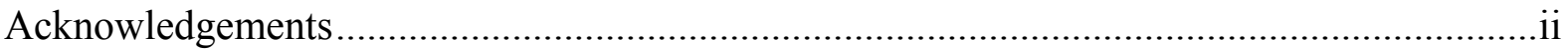

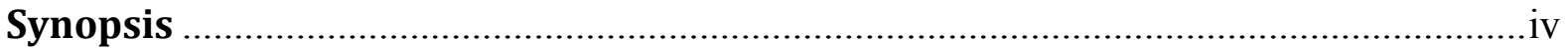

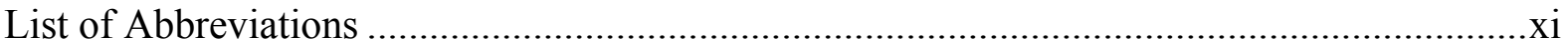

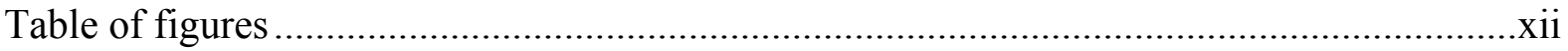

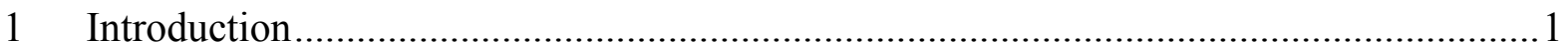

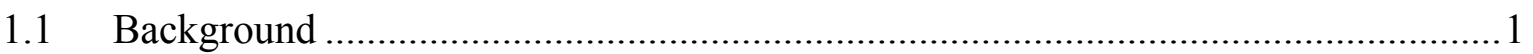

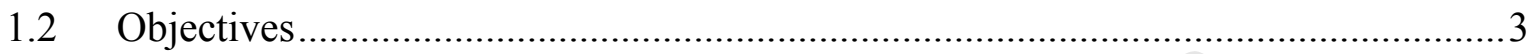

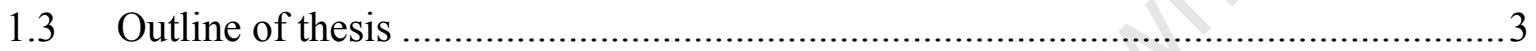

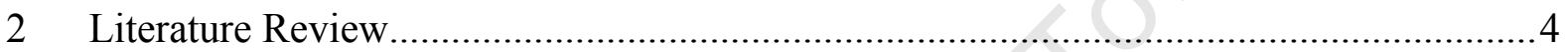

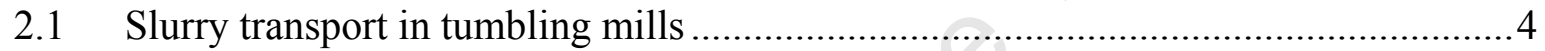

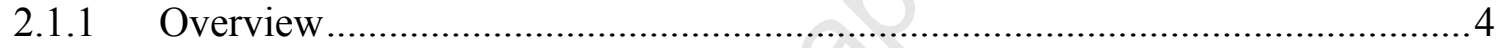

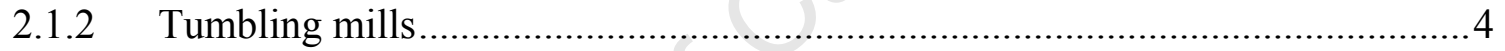

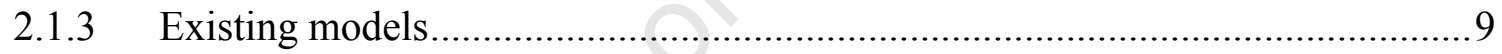

2.1.4 Numerical modelling of slurry transport................................................... 13

2.1.5 The effect of mill operating parameters on slurry transport ........................... 14

2.1.6 Fluid transport in porous media: Sensitivity of porosity …............................ 17

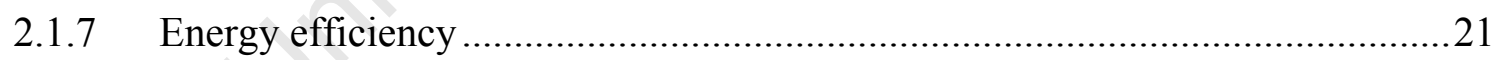

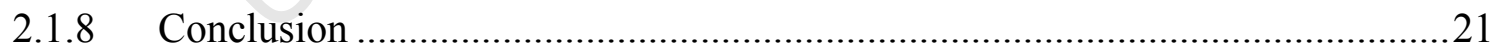

2.2 Particle packing in rotating drum systems .......................................................22

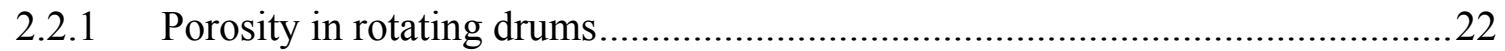

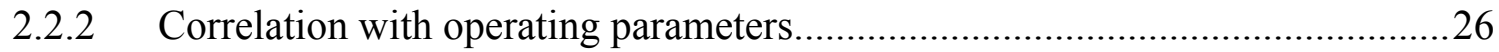

2.2.3 Porosity of multi-component mixtures in rotating drums ................................33

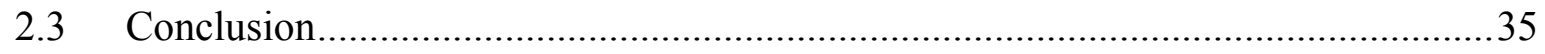

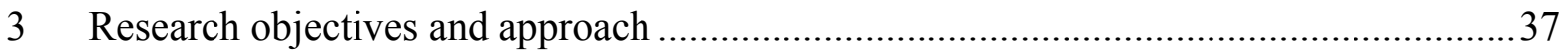

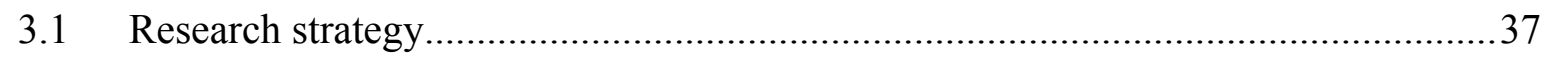




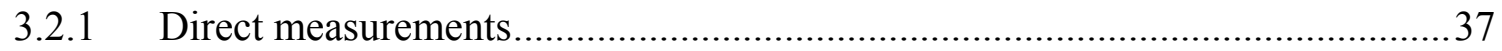

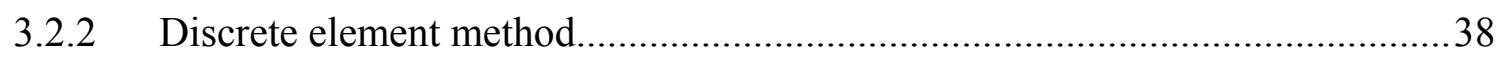

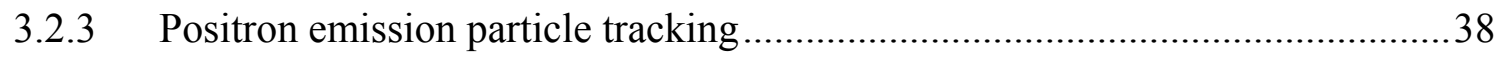

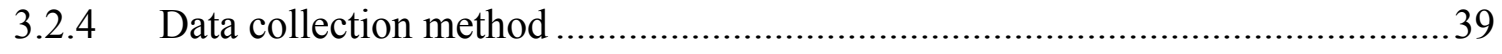

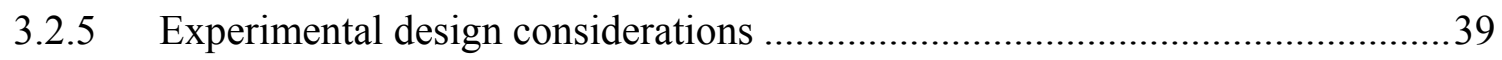

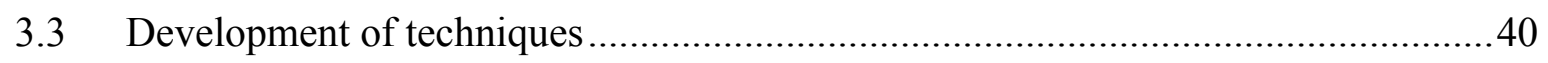

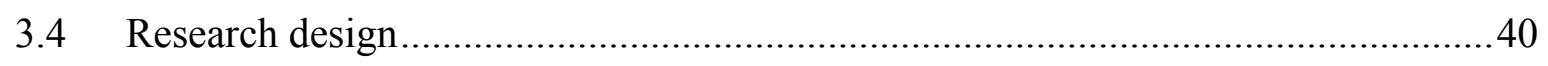

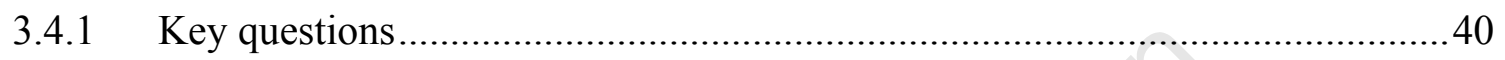

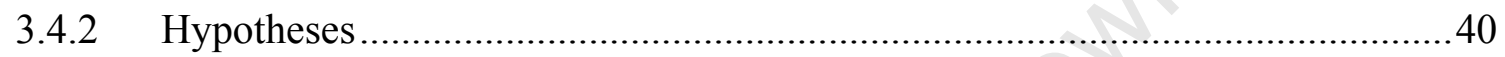

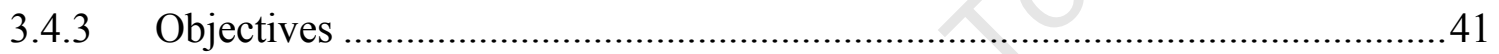

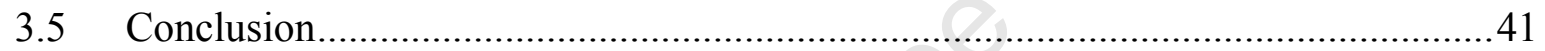

4 Positron Emission Particle Tracking....................................................................42

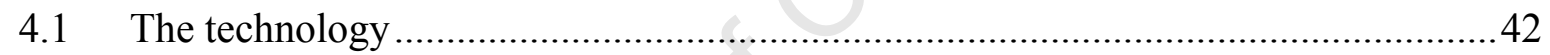

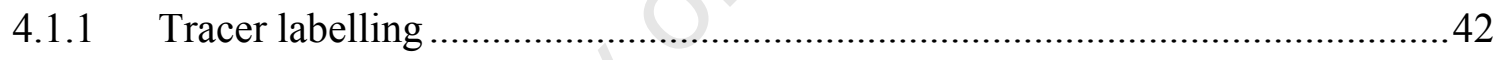

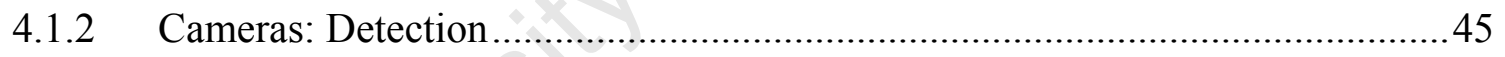

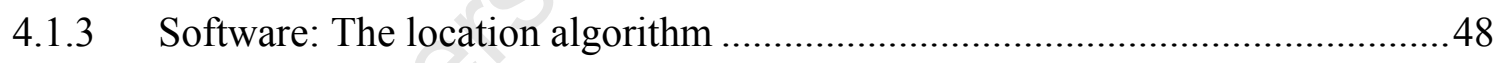

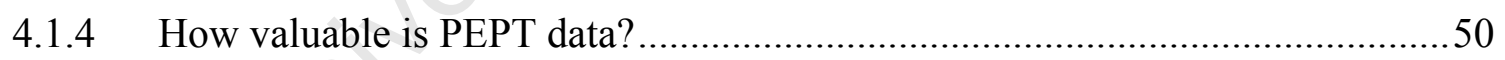

4.2 Packing characterisation using single particle data.............................................51

4.2.1 Defining measures through single particle tracking ........................................51

4.2.2 Calculating the packing fraction of a monosized system.................................52

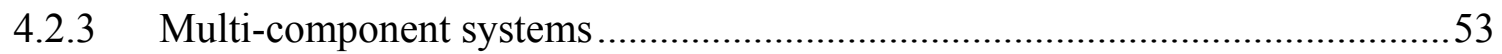

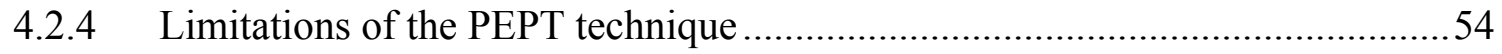

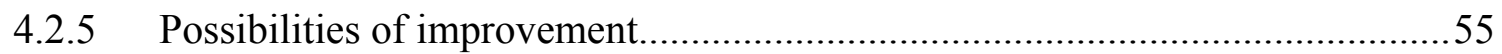

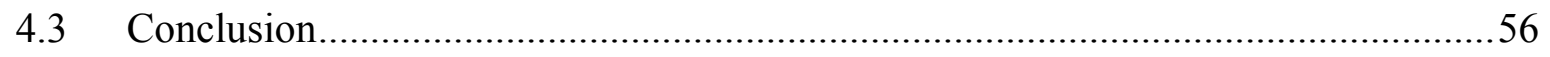

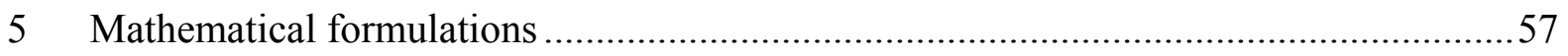

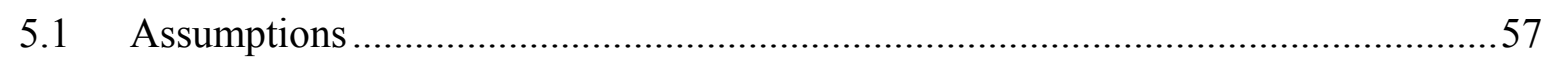




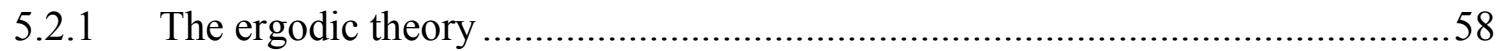

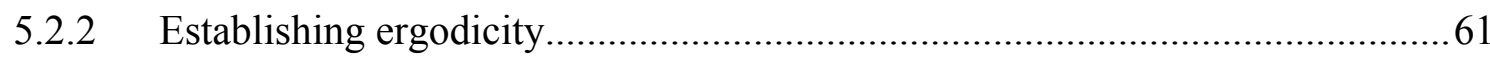

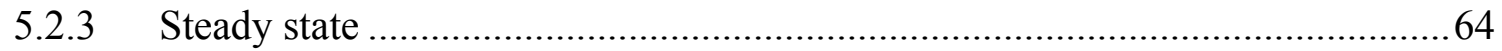

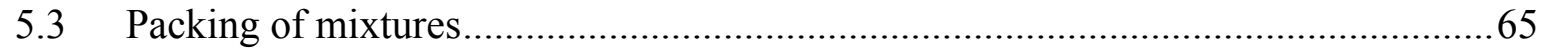

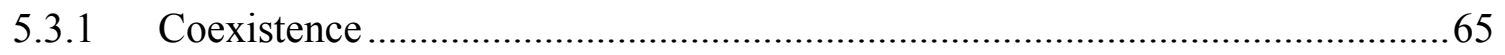

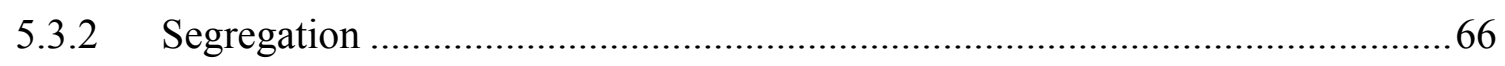

5.3.3 Tracking a particle per size component ........................................................69

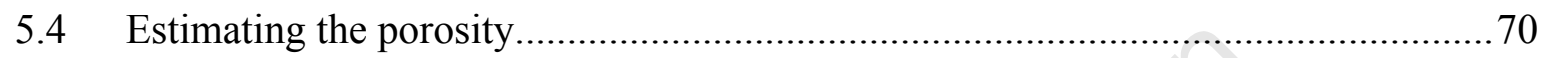

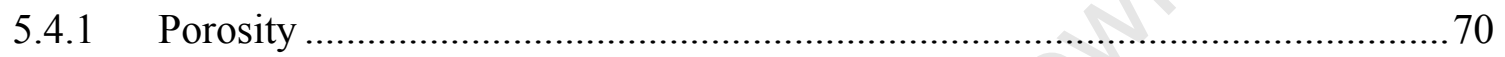

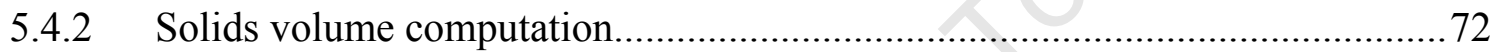

5.4.3 Number of particles in a given volume element ........................................... 72

5.4.4 Combining the volumes of solid from different size components ....................74

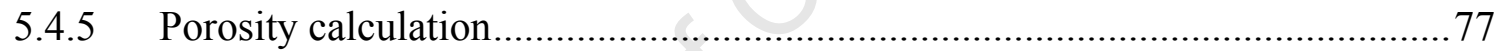

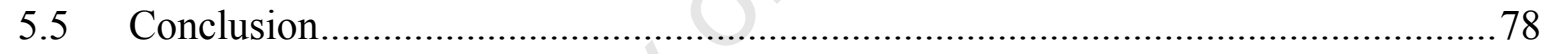

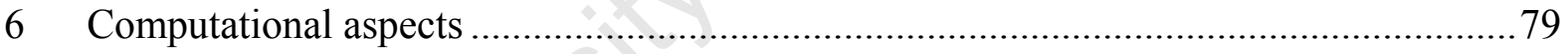

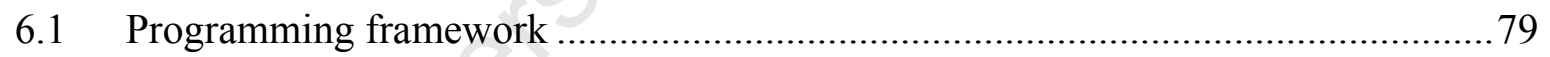

6.2 Number of particles in a size component .................................................... 79

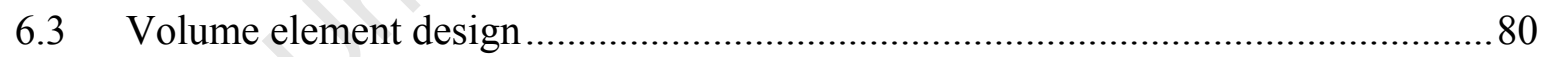

6.4 Computation of residence time fraction ............................................................ 81

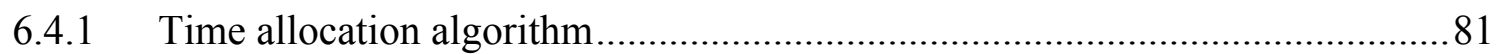

6.4.2 Time-difference allocation to volume elements ........................................... 82

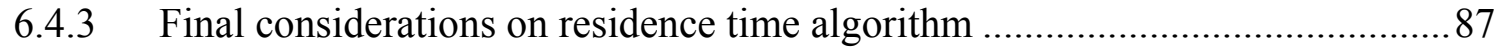

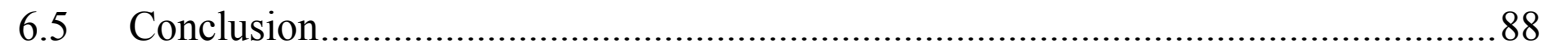

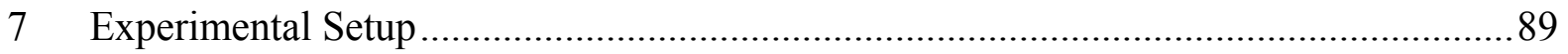

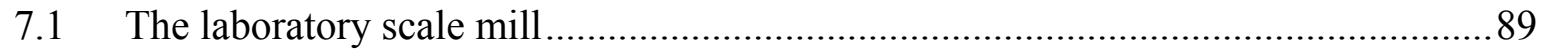

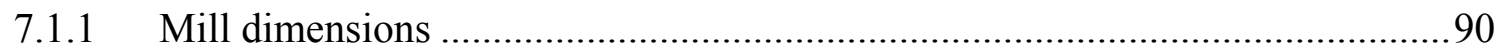




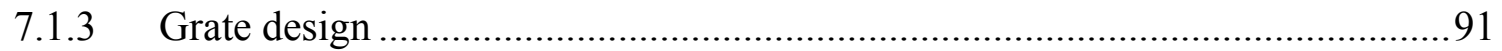

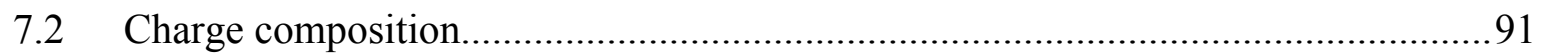

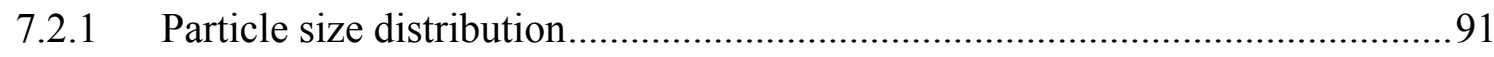

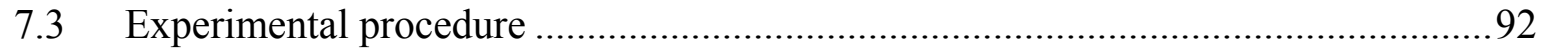

7.3.1 Positioning the mill between the cameras....................................................... 92

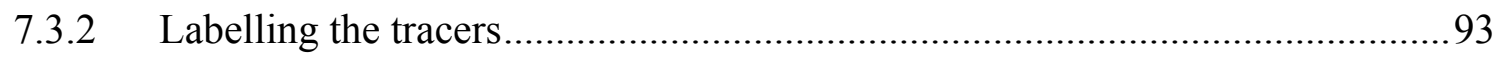

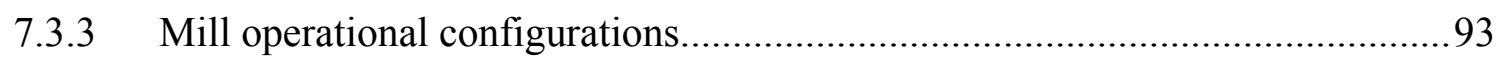

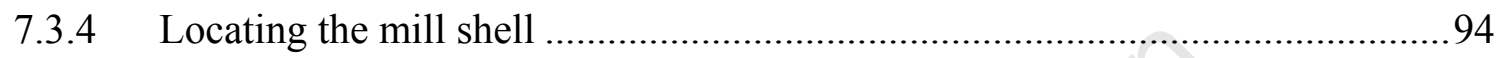

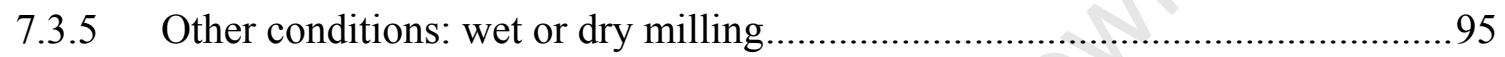

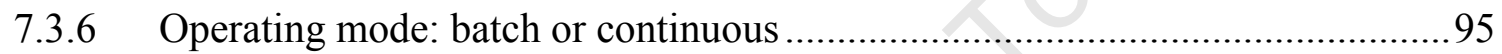

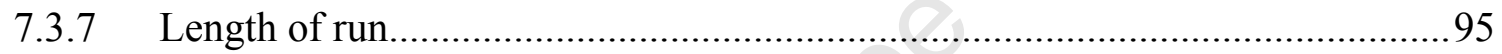

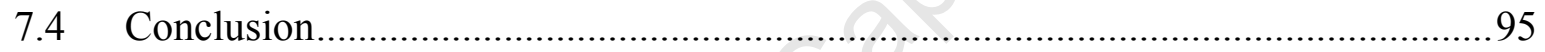

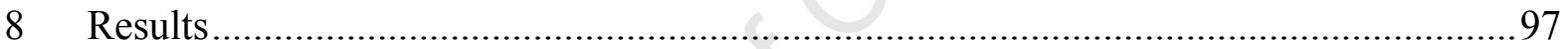

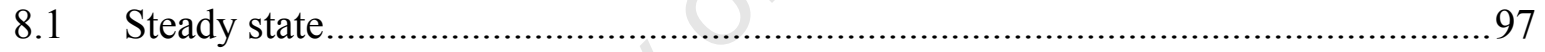

8.2 The effect of speed on porosity distribution....................................................... 98

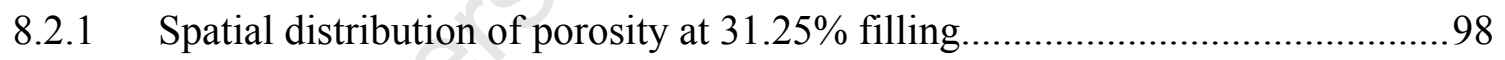

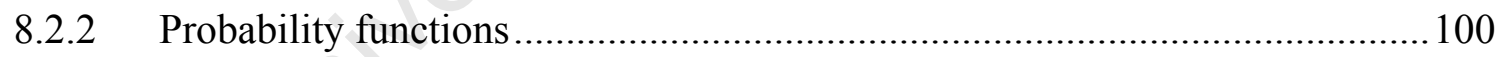

8.3 Average porosity vs Mill rotation speed .......................................................... 102

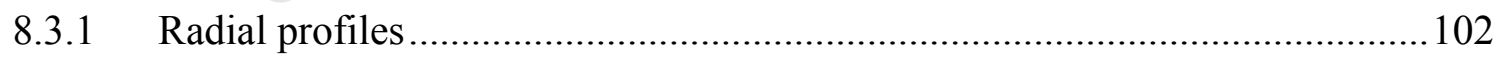

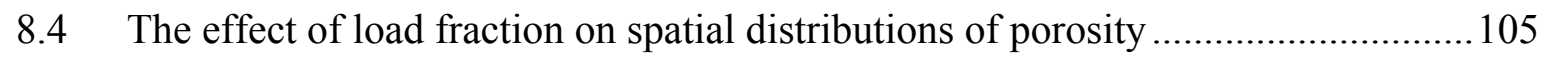

8.5 The combined effect of mill speed and load fraction on porosity........................... 106

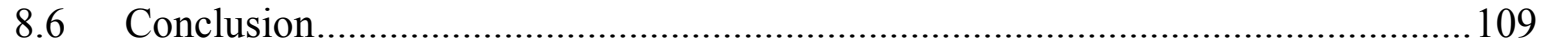

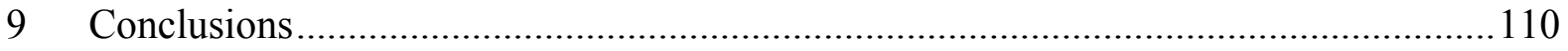

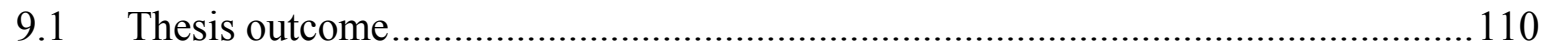

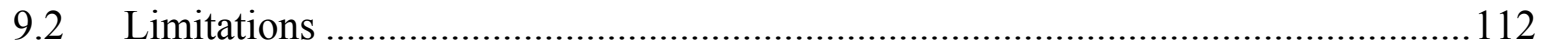

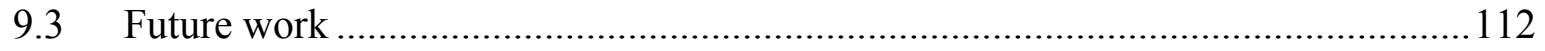


REFERENCES ...

114 


\section{List of Abbreviations}

CoM Centre of Mass

DC Direct Current

DEM Discrete Element Method

HDPE High Density Polyethylene

LOR Line of Response

PEPT Positron Emission Particle Tracking

PET Positron Emission Tomography

SRC standardised Regression Coefficient 


\section{Table of figures}

Figure 2-1 Tumbling mill (Radziszewski et al., 2005) .........................................................5

Figure 2-2 Schematic of a typical SAG mill (Latchireddi and Morrell 2003)........................5

Figure 2-3 Principal breakage mechanisms (Napier-Munn et al., 1996) ................................6

Figure 2-4 Ideal slurry flow in a typical grate discharge mill (Latchireddi and Morrell 2006) 8

Figure 2-5 Stages of transportation in a grate discharge mill (Latchireddi and Morrell 2006).8

Figure 2-6 Schematic of laboratory mill used by Morrell and Stephenson (1996) .................12

Figure 2-7 Schematic of observed flow out of the grate at different rotational speeds ...........16

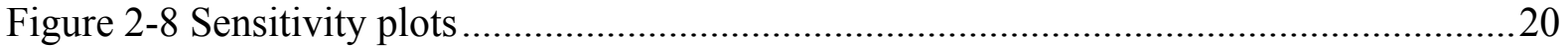

Figure 2-9 Different flow regimes represented graphically..............................................23

Figure 2-10 Residence time distributions of tracer at different configurations. Set A: $1.5 \mathrm{~mm}$ beads in $136 \mathrm{~mm}$ diameter drum; Set B: $3 \mathrm{~mm}$ beads in $144 \mathrm{~mm}$ diameter drum; and Set C:

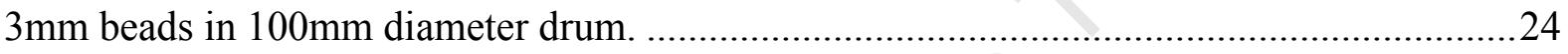

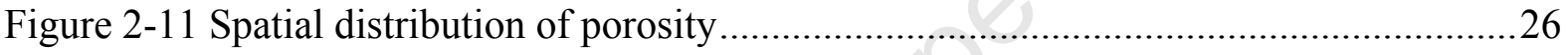

Figure 2-12 Porosity as a function of rotation speed: probability density distribution (Yang et al., 2003) .27

Figure 2-13 Average porosity as a function of rotation speed (Yang et al., 2003)................28

Figure 2-14 Spatial distribution of porosity at different speeds: a) 5rpm; b) 80rpm; c) 150rpm; d) $200 \mathrm{rpm}$ .29

Figure 2-15 Distributions of packing density at different speeds (Yang et al., 2008) .............30

Figure 2-16 Mean packing density at different rotation speeds (Yang et al., 2008)...............31

Figure 2-17 Effect of drum fill on the surface shape of the particle bed (Santomaso et al. 2003)

Figure 2-18 Analysis of the bed properties for mill speeds in: a) the rolling regime (1.57 $\mathrm{rad} / \mathrm{s})$; b) the cascading regime $(12.56 \mathrm{rad} / \mathrm{s}) ; \mathrm{c})$ cataracting regime $(15 \mathrm{rad} / \mathrm{s})$ and; d)

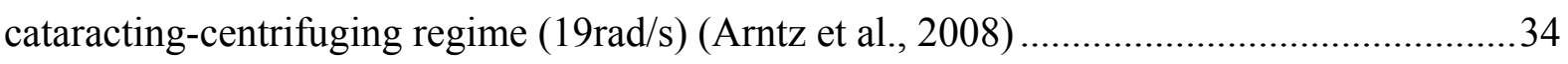

Figure 4-1 Effect of $\mathrm{Fe}^{3+}$ on ${ }^{18} \mathrm{~F}$ adsorption on quartz (Initial ${ }^{18} \mathrm{~F}$ concentration: $6.8 \times 10^{9} \mathrm{M}$ )

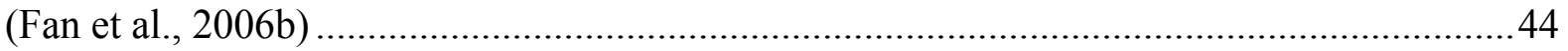

Figure 4-2 The new Birmingham positron camera (Parker et al., 2002) ...............................46

Figure 4-3 The convergence of lines at a centroid region representing particle position........49

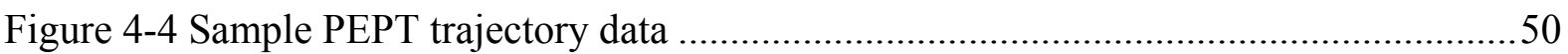

Figure 5-1 Segregation mechanisms: (a) segregation by percolation only; (b) segregation by buoyancy only; (c) segregation when percolation and buoyancy act together; and (d) 
segregation when percolation and buoyancy oppose each other. Symbol fill: open - lower density particles; filled - higher density particles.

Figure 5-2 Occupancy plot of different sized tracers in a binary system (Fan et al., 2006a) ..67

Figure 5-3 Segregation demonstrated by occcupancy of small (a) and large (b) particles (Ding

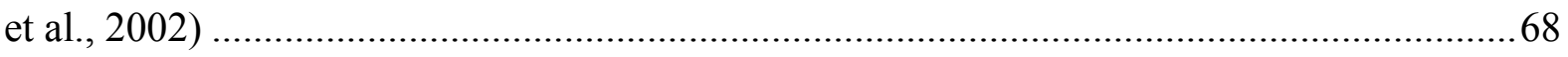

Figure 5-4 Contour maps showing the packing density of different sized particles...............69

Figure 5-5 Schematic of particles in a multi-component mixture in a volume element..........75

Figure 5-6 Volume fraction of small and large particles in a cylinder for a volume filling

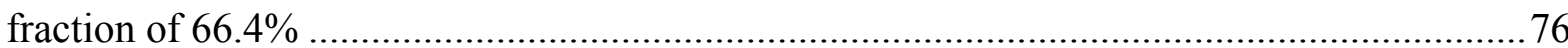

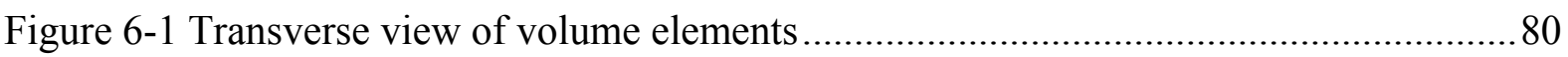

Figure 6-2 Diagram showing the allocation of time based on distance $d_{j}$ the tracer covers in a

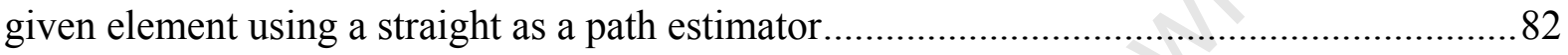

Figure 6-3 Similar triangles with ratios given as follows $\mathrm{AB} / \mathrm{AC}=\mathrm{AE} / \mathrm{AD}=\mathrm{BE} / \mathrm{CD}$.........83

Figure 6-4 Straight line estimate of the path a particle takes across volume elements ..........84

Figure 6-5 Comparing time sharing methods: line and curve............................................ 86

Figure 7-1 Schematic experimental mill used in the experiments ........................................89

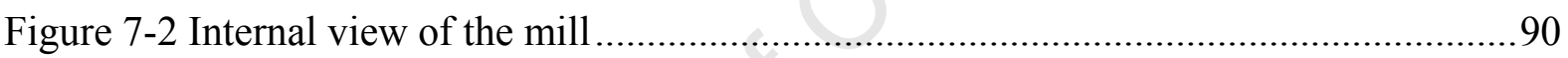

Figure 7-3 Schematic of mill shell outline and magnified view of single lifter ....................91

Figure 7-4 Truncated Rosin-Rammler size distribution of glass beads ................................92

Figure 7-5 The experimental mill placed between detectors ............................................93

Figure 7-6 Tracer placed on mill shell for locating the mill shell .......................................94

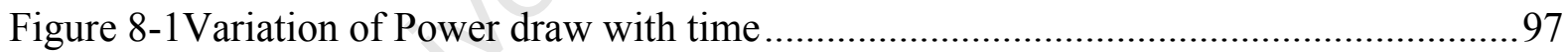

Figure 8-2 Spatial distribution of porosity at different mill speeds: A) 60 B) 70 C) 75 D) 80

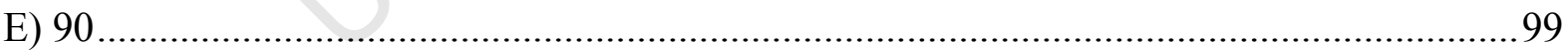

Figure 8-3 Distribution of porosity values at different mill rotation speeds......................... 101

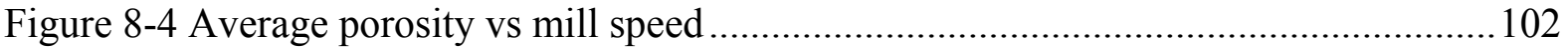

Figure 8-5 Relevant region when computing radial profiles ............................................ 103

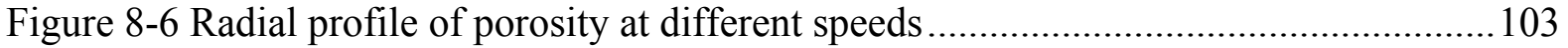

Figure 8-7 Variation of spatial distribution of porosity with load fraction........................... 105

Figure 8-8 Spatial distribution plots showing the combined effect of mill speed and load fraction on porosity 


\section{Introduction}

\section{$1.1 \quad$ Background}

Porosity is a simple but important quantity in the characterisation of porous media. It is defined as the fractional void volume of a given porous system. This quantity affects key processes within various systems. In rotary mills, which were the primary focus of this study, it affects processes such as fluid transport and abrasion. Rotary mills are very important devices in science and engineering. They have wide applications in the processing industry, including: pharmaceuticals, food processing and mineral processing. In mineral processing, they are used for comminution. Comminution is the process of reducing ore size for the purpose of mineral liberation. In this process, ore is crushed and ground to sizes that can easily be used in subsequent processes, such as flotation, leaching and smelting.

There are many devices that are used in comminution. Of these, tumbling mills are amongst the most commonly used - for grinding in particular. Tumbling mills are horizontal drums partially filled with ore and rotated about their horizontal axes. The ore comprises what is known as charge. Due to the rotational motion of the mill, a grinding field is formed-at sufficient speeds - in which ore breakage occurs. The end-product of this process is passed on to other equipment, downstream, for finer grinding.

Grinding is an energy-intensive operation which has a lot of room for improvement. To optimise mill operations requires a good understanding of key processes within the mill. These processes include grinding and material transport. Material transport or entrainment of the end-product of grinding, across and out of the mill, is particularly inefficient. In fact, the lack of understanding of this mechanism in tumbling mills is the main bottleneck in trying to improve grinding circuit efficiency (Songfack and Rajamani, 1999).

Tumbling mills may be operated in "wet grinding" or "dry grinding" mode. In wet grinding, the agent of entrainment is water, which is fed into the mill on the same end as the ore. Water, when mixed with fine particles, forms a complex fluid called slurry. The slurry then flows through the pore network in the rotating charge and out of the mill. To attain optimal performance, sufficiently fine particles must be removed from the mill as quickly as possible, after being generated (Cleary et al., 2006). Hence, slurry transport is a key factor affecting 
mill performance. This justifies the need for a better understanding of slurry transport, which is very poorly understood.

To optimise slurry transport requires adequate knowledge and characterisation of the factors affecting it. Key to understanding fluid transport in porous media is packing porosity. In fact, the packing porosity of the dynamic charge has been found to be a key variable affecting mill performance (Latchireddi and Morrell, 2003). Packing porosity in this context refers to how much voids exist among particles, as opposed to the voids within particles. For brevity, however, the term porosity implies packing porosity throughout this thesis.

Despite porosity being so markedly important, it is very poorly understood in tumbling mills - and rotary mills, in general. Most existing models of slurry transport are empirical and assume a uniform porosity (in tumbling mills) of 0.4. This uniformity assumption is inconsistent with evidence from studies of particle packing done in rotating drums (Yang et al., 2003, 2008; Arntz et al., 2008). The models, therefore, are not only difficult to extrapolate beyond experimental conditions, but also inaccurate.

The studies cited above are based on simplified systems that do not depict typical conditions in real mills. For example, most of the work has been on simple rotating drums that do not have lifters. The studies are also based on monosized systems and binary systems. In some studies, operating conditions such as mill speed and filling fraction are outside the range of those typical of tumbling mills. Filling fraction refers to the percentage of the mill internal volume occupied by the charge. To the best of my knowledge, there is no study of porosity that fully incorporates design and operating conditions comparable to those of tumbling mills. Therefore, the understanding of porosity in rotary mills is still insufficient.

It is against this background that a study of the spatial distribution of charge porosity in tumbling mills has been undertaken. It should be noted, however, that previous studies have significantly improved our understanding of particle packing in rotary mills. This note comes with an understanding of the difficulty with which such studies have been conducted, owing to the aggressive environment within such systems. Coupling these complexities with the size of tumbling mills explains why simpler systems have been studied.

In this study, one goal was to include some key features of tumbling mills, such as lifters, discharge grate and multi-component charge mixtures. It includes investigating how porosity 
varies with mill operating parameters. The primary aim has been to contribute to improved understanding of slurry transport and to mill optimisation.

This study is expected to improve our understanding of porosity in rotary mills, in general; hence the title: "Characterising the porosity of multi-component mixtures in rotary mills".

\subsection{Objectives}

The goal of this thesis was to characterise porosity in rotary mills. Subsequently, the aim has been to develop techniques for measuring porosity (and other packing characteristics) of multi-component mixtures in rotary mills. The study is based on a laboratory scale mill under realistic tumbling mill conditions; particularly, mill speed and filling fraction. For simplicity, however, the charge is composed of glass beads.

The method of measurement used in this study is positron emission particle tracking (PEPT). PEPT entails following the motion of a single radioactively labelled particle (the tracer). If studied for a sufficiently long period, the tracer's trajectory can be used, under certain assumptions, to determine information about the whole system. However, since only one particle is tracked, there must be a way to transform single-particle data to information about the whole system. Fortunately, methods exist that provide this link for monosized systems. Extending such methods to include multi-component mixtures is a major step towards understanding these systems. This problem comprised a significant part of this thesis.

Therefore, mathematical formulations for studying multi-component mixtures using single particle trajectory data are proposed. The formulations once developed, are used in characterising key features of porosity in rotary mills. The results of this process are key inputs into mechanistic modelling of slurry transport in rotary mills.

\subsection{Outline of thesis}

The next chapter is a review of studies of slurry transport and porosity in rotary mills. Chapter three discusses the research design and other methodological aspects. Chapter four gives an account of the PEPT technique and how it is useful in the study of porosity. Chapters five and six discuss the formulation of techniques for porosity calculation. The seventh chapter is an account of the experimental set up. The results and discussions are presented in chapter eight. Finally, conclusions and recommendations are given in chapter nine. 


\section{Literature Review}

This chapter presents a review of relevant literature in the study of porosity in rotary mills. It has two major parts. The first presents the motivation for this work based on a study of slurry transport through the rotary mill charge and transport in unconsolidated porous media. However, to understand slurry transport requires studying porosity in rotary mills. Therefore, the second part of this chapter is dedicated to studies of porosity in rotary mills and similar systems. Through these studies, the reader should observe that existing models of porosity in rotary mills are still inadequate. Finally, the conclusion recounts the need to develop techniques for measuring the porosity of multi-component mixtures in dynamic systems using accurate measurement techniques.

\subsection{Slurry transport in tumbling mills}

\subsubsection{Overview}

To understand the need for this work, slurry transport is investigated. Firstly, a brief background on tumbling mills is given. This is followed by a discussion on existing models of slurry transport. To further demonstrate the importance of porosity to slurry transport modelling, the transport of fluids in unconsolidated porous media is also reviewed, briefly.

\subsubsection{Tumbling mills}

\subsubsection{The equipment}

Tumbling mills are very important equipment used in the comminution of ore in mineral processing plants. Comminution entails the process of reducing ore size for the purpose of mineral liberation. Figure 2-1 shows the external view of an industrial scale tumbling mill installed in a mineral processing plant. 


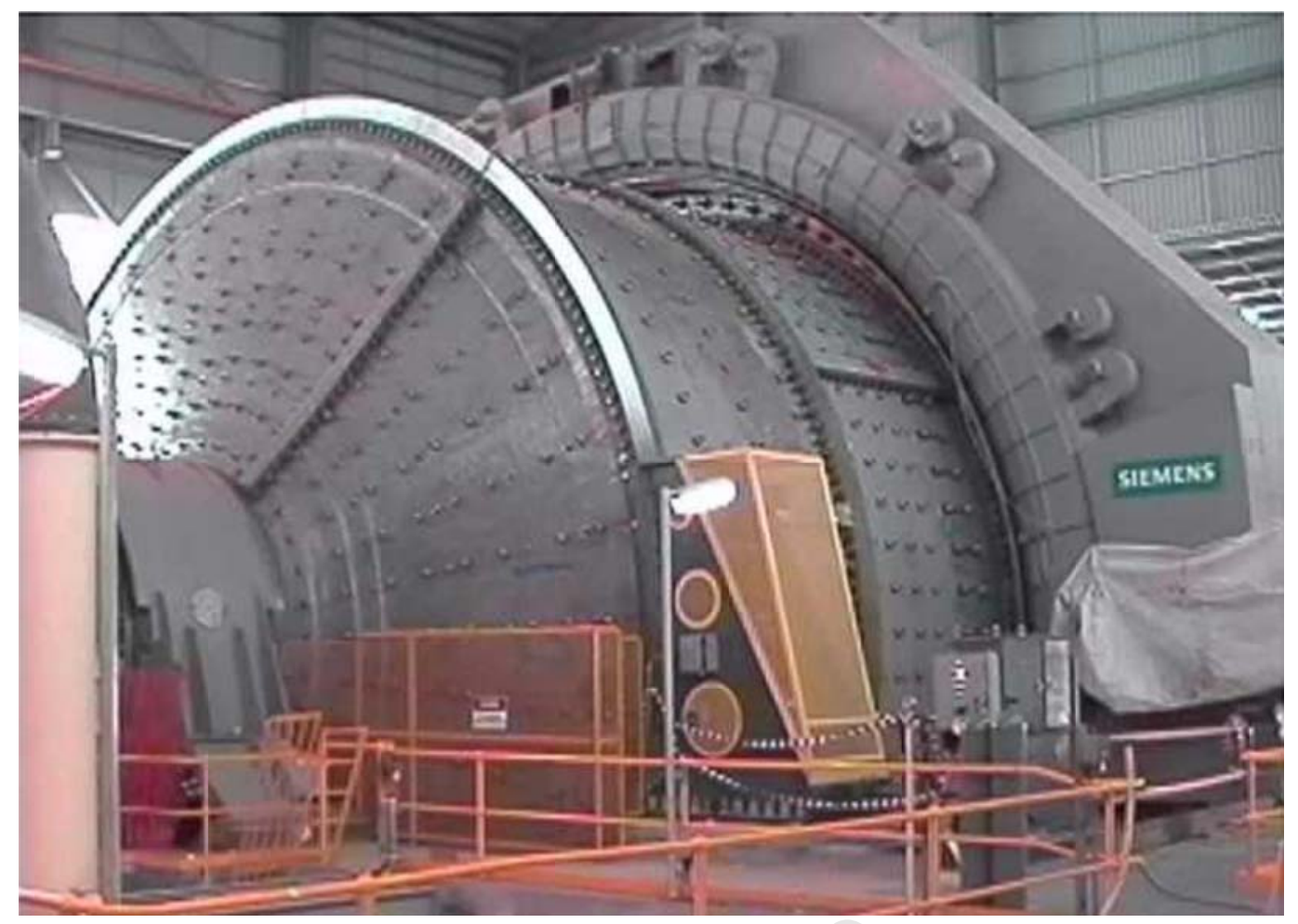

Figure 2-1 Tumbling mill (Radziszewski et al., 2005)

Tumbling mills are composed of three main elements: the mill shell, lifters and charge. Other key features are the discharge grate which separates the main mill from the pulp chamber. The mill has two ends for feed and discharge. Figure 2-2 is a schematic view showing key features of the mill.

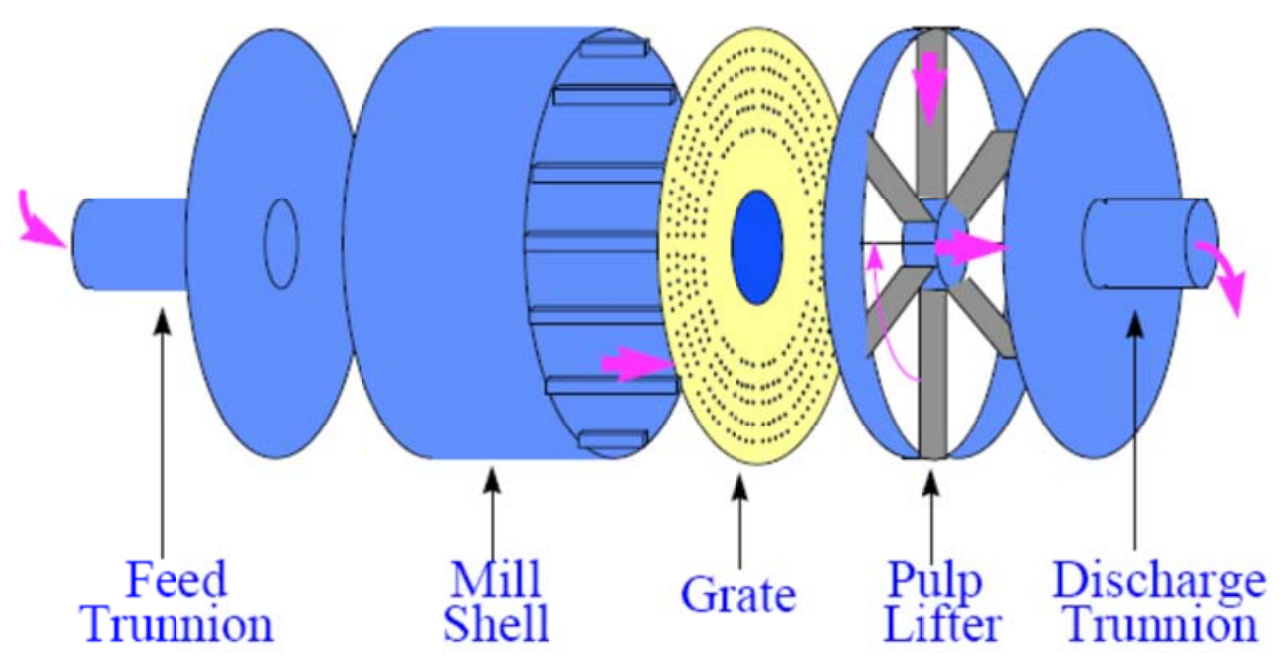

Figure 2-2 Schematic of a typical SAG mill(Latchireddi and Morrell 2003) 
The lifters can be seen along the lining of the mill shell. The arrows show the path taken by water as it flows into the main milling chamber, mixing with the fine particles to form slurry, through the discharge grates and out of the mill. Slurry transport will be discussed shortly.

Tumbling mills can be classified, according to the grinding media used, as either autogenous (AG) or semi-autogenous (SAG). In AG mills the ore forms the grinding media. SAG mills, on the other hand, are also charged with steel balls which form part of the grinding media. Tumbling mills also vary in lifter design, aspect ratio (or length-to-diameter ratio) and discharge grate design.

\subsubsection{Breakage and material transport}

There are two key processes that take place in tumbling mills, namely: breakage and material transport. Thus, the mill can be viewed as both a grinding machine and a pump. To start with, ore that requires grinding is fed into the mill through the feed trunnion. The feed is usually a product of the crushing process and is approximately $25-300 \mathrm{~mm}$ in size. Crushers are used for crushing.

When the mill is in operation, the motion of the mill shell and the action of the lifters induce motion in the charge. At appropriate rotation speeds, a grinding field is formed. Breakage usually occurs within this field. There are three breakage mechanisms: impact, abrasion and attrition. These are illustrated in Figure 2-3.

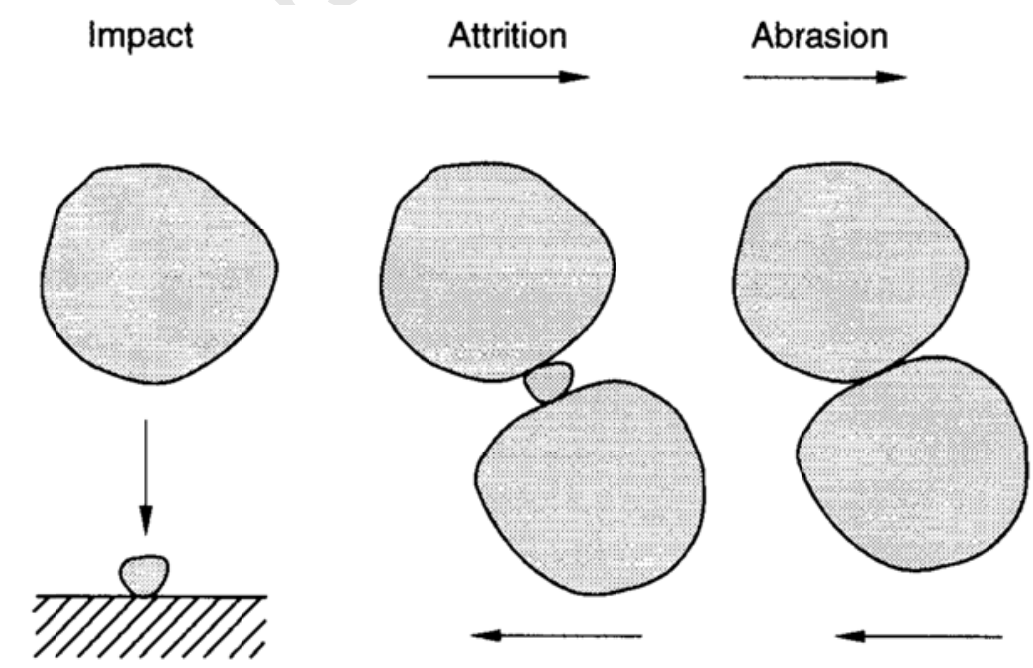

Figure 2-3 Principal breakage mechanisms (Napier-Munn et al., 1996)

The description given here is mainly based on Napier-Munn et al. (1996). The impact mechanism of breakage occurs when particles collide more or less head on. It may also 
involve collision between a particle and the mill shell. Attrition occurs when a particle (usually smaller) is squeezed between other particles (or between another particle and the shell). The two agents of attrition move in opposing directions, as shown in Figure 2-3. In abrasion, two particles moving in opposing direction rub against each other, as shown in the figure above. Breakage may occur at once upon a particle encountering any of the above situations. On the other hand, it may be incremental, in which case particles encounter a succession of any of the three situations in Figure 2-3, resulting in breakage.

The end-product of milling is fine particles, approximately 38-2000 $\mu \mathrm{m}$. These can be transported towards the discharge end by several methods, including particle dynamics, diffusion and slurry flow (Cleary et al., 2006). Thus, the transport process may result from the combined effects of gravity, fluid flow, and the mechanical action of the rotating mill and tumbling charge.

In wet grinding, the agent of entrainment is water. The water is fed into the mill through the feed trunnion. When it mixes with fine ore particles a complex fluid called slurry is formed. The slurry is transported through the porous charge and then out of the mill, through the discharge trunnion. Most $\mathrm{AG}$ and $\mathrm{SAG}$ mills are operated in this way. Therefore, understanding slurry transport is essential, because it affects mill performance greatly.

\subsubsection{The flow of slurry}

Despite the motion of the charge and the slurry being predominantly rotational, the slurry generally flows along the horizontal axis. Furthermore, as water and ore are continually fed into the mill, a slurry pressure gradient is formed across the mill. This results in axial transport towards the discharge end. Figure 2-4 below depicts the flow of slurry through the mill. 


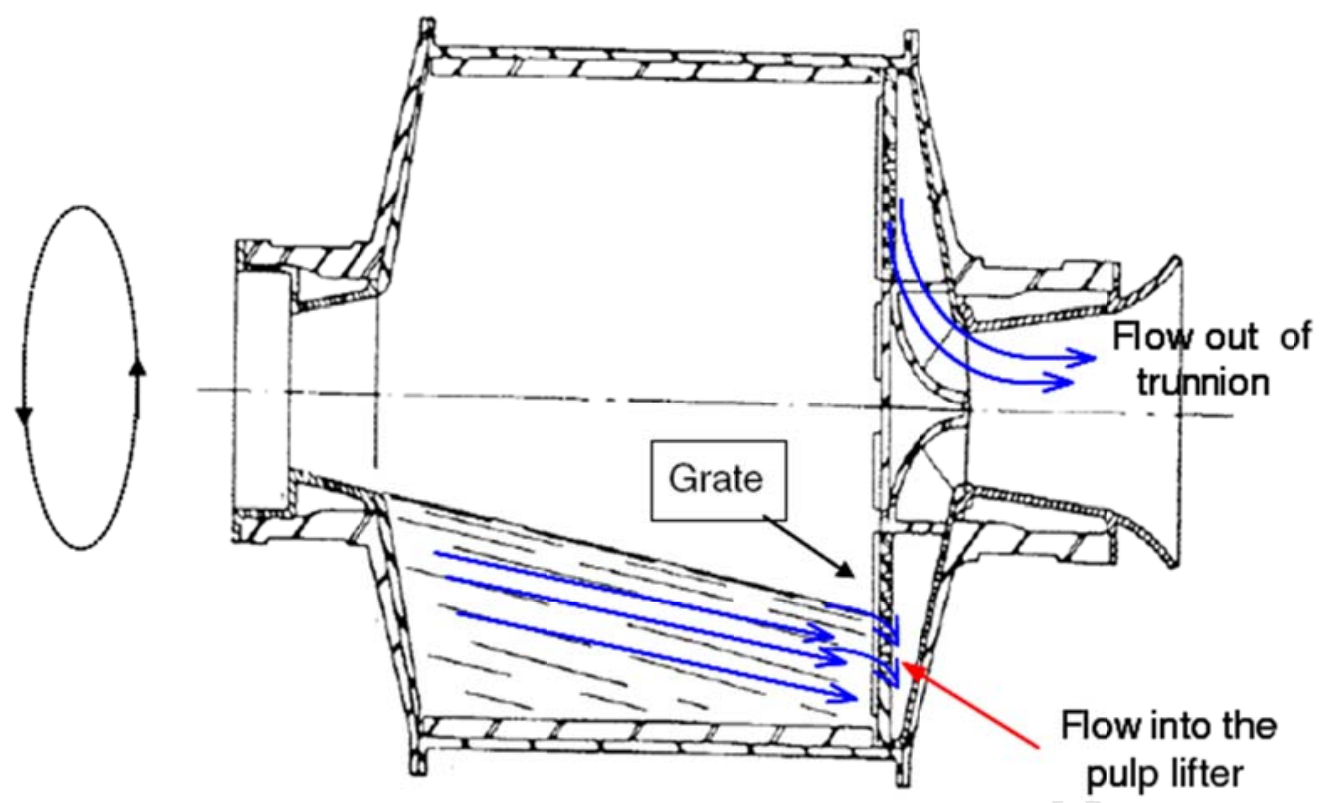

Figure 2-4 Ideal slurry flow in a typical grate discharge mill (Latchireddi and Morrell 2006)

The blue arrows show the typical axial path the slurry takes, from the feed trunnion to the discharge trunnion. It is important to note that a large portion of slurry actually flows through the porous network within the rotating charge. On the other hand, a significant amount of slurry flows through the pool that may be formed at the toe region of the mill. After making its way through the mill charge and/or pool, the slurry then proceeds to the pulp chamber, through the discharge grate. The pulp chamber usually consists of pulp lifters, which lift the slurry and directs it into the discharge trunnion, and finally out of the mill. A summary of this process is shown schematically in Figure 2-5.

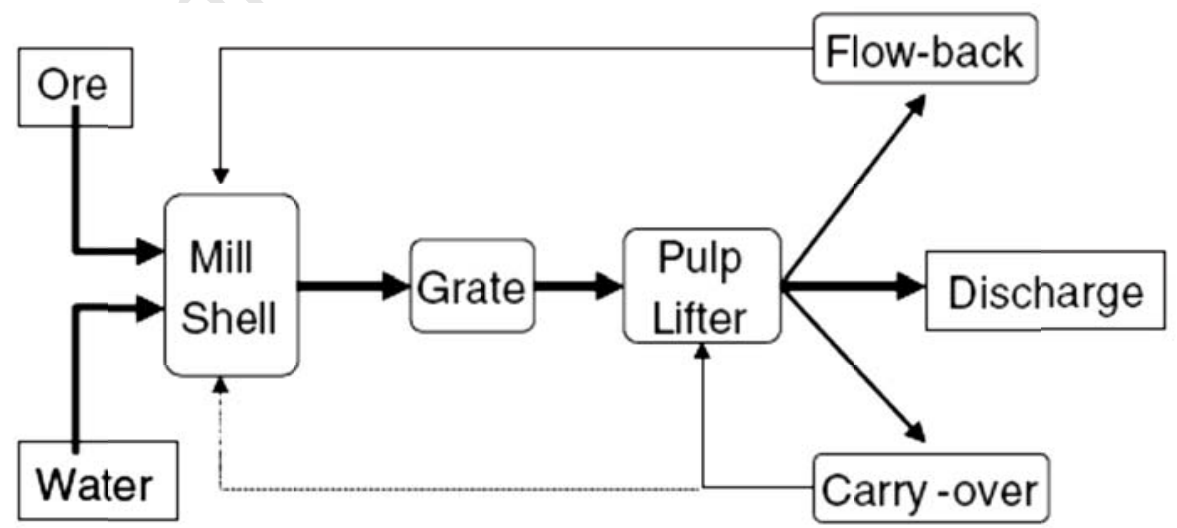

Figure 2-5 Stages of transportation in a grate discharge mill (Latchireddi and Morrell 2006)

Of primary interest in this work is how slurry is transported through the porous charge. This is essential to understanding the whole transport process. The process is definitely more complex 
than the description given above. Nonetheless, understanding slurry transport in tumbling mills requires knowledge of both slurry properties and the pore structure through which the slurry flows. Consequently, many studies have been conducted on slurry transport, some of which are discussed shortly.

\subsubsection{Importance of slurry transport modelling}

To achieve optimal mill performance and throughput, the removal of sufficiently milled particles must be efficient. Thus, slurry transport greatly affects mill grinding capacity and performance. Inadequate slurry removal results in many known problems, which include the following (Cleary et al., 2006):

- Charge lubrication. Too much slurry constrains breakage resulting from cascading motion by causing a reduction in frictional energy transferred to the charge.

- Flow back. The slurry that flows through the discharge grate may flow back into the mill charge once the pulp lifters have risen above the free surface of the charge.

- Slurry pooling. A slurry pool may form at the toe of the charge if slurry removal is inadequate. This can constrain breakage if it rises into the impact zone.

Considering the last point above, it should be noted that, it is the saturation of the interstices within the charge that usually leads to the formation of the slurry pool. The issues raised above also underscore the need to understand slurry transport and factors that affect it. To achieve optimal slurry transport - and overcome the problems above-requires modelling of slurry transport. Models, if appropriately constructed, provide a way of predicting performance for given mill operating parameters. They can also help in studying systems based on hypothetical conditions and designs. Thus, using models, it is possible to find a set of operating conditions that correspond to optimal mill efficiency.

\subsubsection{Existing models}

This section discusses some studies of slurry transport modelling conducted to date. It focuses on slurry transport through the charge. The aim is to explore opportunities for improved operations, especially due to improved understanding of charge properties.

Most studies of slurry transport through the charge have been empirical. These have barely included analysis of slurry motion at the particle scale. In most cases, the focus has been on 
design and mill operation parameters, without direct consideration of how slurry actually flows through the charge. Consequently, the models are difficult to extrapolate beyond experimental conditions.

For extrapolation to be feasible, understanding how changing mill operating parameters affect charge permeability at particle level is vital. In particular, there is need for adequate knowledge of charge properties and how these relate with mill operating conditions. This would make the development of a mechanistic transport model possible. In this section, a number of empirical and semi-empirical studies of slurry transport conducted to date are discussed.

Hogg and Rogovin (1982) developed a model for transport of particles in a wet overflow ball mill based on analysis of physical processes which occur in the mill. However, in developing their model, they assumed that slurry transport occurs only in the slurry pool. They also assumed that the interstices in the charge are completely filled with slurry at all times. This model may not always be applicable because the two assumptions do not always hold. The assumption that the interstices in the charge are always saturated may only apply to overflow mills. It is also clear that slurry transport occurs in both the slurry pool and charge (Moys, 1986). Other studies on overflow ball mills include the work by Klimpel et al. (1989).

Moys (1986) followed a mechanistic approach in the development of a slurry transport model. The author described the effect of slurry viscosity on flow through the charge using chemical engineering equations for fluid transport through stationary packed beds. Due to the mechanistic approach followed, the model had only three arbitrary parameters that had to be determined by experiment. For that reason, the author stated that the model could be used fairly safely for extrapolation beyond the range of experimental conditions considered. In order to avoid the complex integrals that needed to be evaluated, empirical formulas were developed which allowed approximation of those integrals. Experiments were then used to determine how well the model would describe the dynamic case. The experiments were done on a $0.54 \times 0.83 \mathrm{~m}$ pilot mill.

The flow of slurry through the charge was understood to be due to the axial pressure gradient in the slurry. The gradient is due to a decrease in the slurry level as it flows along the horizontal axis. The pressure gradient was given by the Blake-Kozeny (Bird et al., 1960) equation: 


$$
\frac{d p}{d z}=\frac{-72 \mu \nu(1-\varepsilon)^{2}}{D_{b}^{2} \varepsilon^{3}}
$$

Equation 2.1

where

$v=\varepsilon Q / A$ is the superficial velocity of the fluid;

$D_{b}$ is the average particle diameter in the charge;

$\mu$ is the viscosity;

$\varepsilon$ is the porosity;

$Q$ is the flowrate;

$A$ is the cross-sectional area covered by slurry.

Despite, using the Blake-Kozeny equation, Moys acknowledged that it is only applicable for Reynolds number of less than 30. The author also added that it must be borne in mind that slurry flow is not laminar in nature. In particular, when analysing data obtained in industrial mills, one would need to incorporate the component of pressure drop that depends on $v^{2}$. The volume of slurry in the load is given by:

$$
V=\pi \varepsilon R\left\{\left(h_{L}^{2.5}+2.5 \beta Q L\right)^{1.6}-h_{L}^{4}\right\} / 8 \beta Q
$$

Equation 2.2

where

$R$ is the mill radius;

$L$ is the mill length;

$H$ is the distance from the axis to the slurry level;

$h=R-H$;

$\beta=1.463 / R^{0.5} \rho k_{Q}$, where $k_{Q}$ is a function of $D_{b}, \mu$ and $\varepsilon: k_{Q} \propto D_{b}^{2} \varepsilon^{3} /\left\{\mu(1-\varepsilon)^{2}\right\}$.

Despite the model being mechanistic, Moys (1986) used empirical formulations to evaluate the integrals. The integrals were quoted as having $\pm 8 \%$ accuracy. Furthermore, the model assumes uniform porosity across the charge. In fact, porosity was never measured at all. Thus, the model cannot be fully mechanistic. The fact that Moys' model was developed from the case of fluid flow through a static bed implies that the accuracy of the model is difficult to 
verify. In addition, one would expect that extrapolation should also be a great challenge. Moys, however, maintained that the model accurately describes the slurry transport phenomenon.

Morrell and Stephenson (1996) constructed a model relating slurry hold-up to mill speed, filling fraction and grate design. They studied a pilot scale mill of diameter $300 \mathrm{~mm}$ and 150 $\mathrm{mm}$ long, fitted with lifters and a transparent grate. However, the results were also compared with data from industrial scale mills. Figure 2-6 shows a schematic of the laboratory mill used in the study.

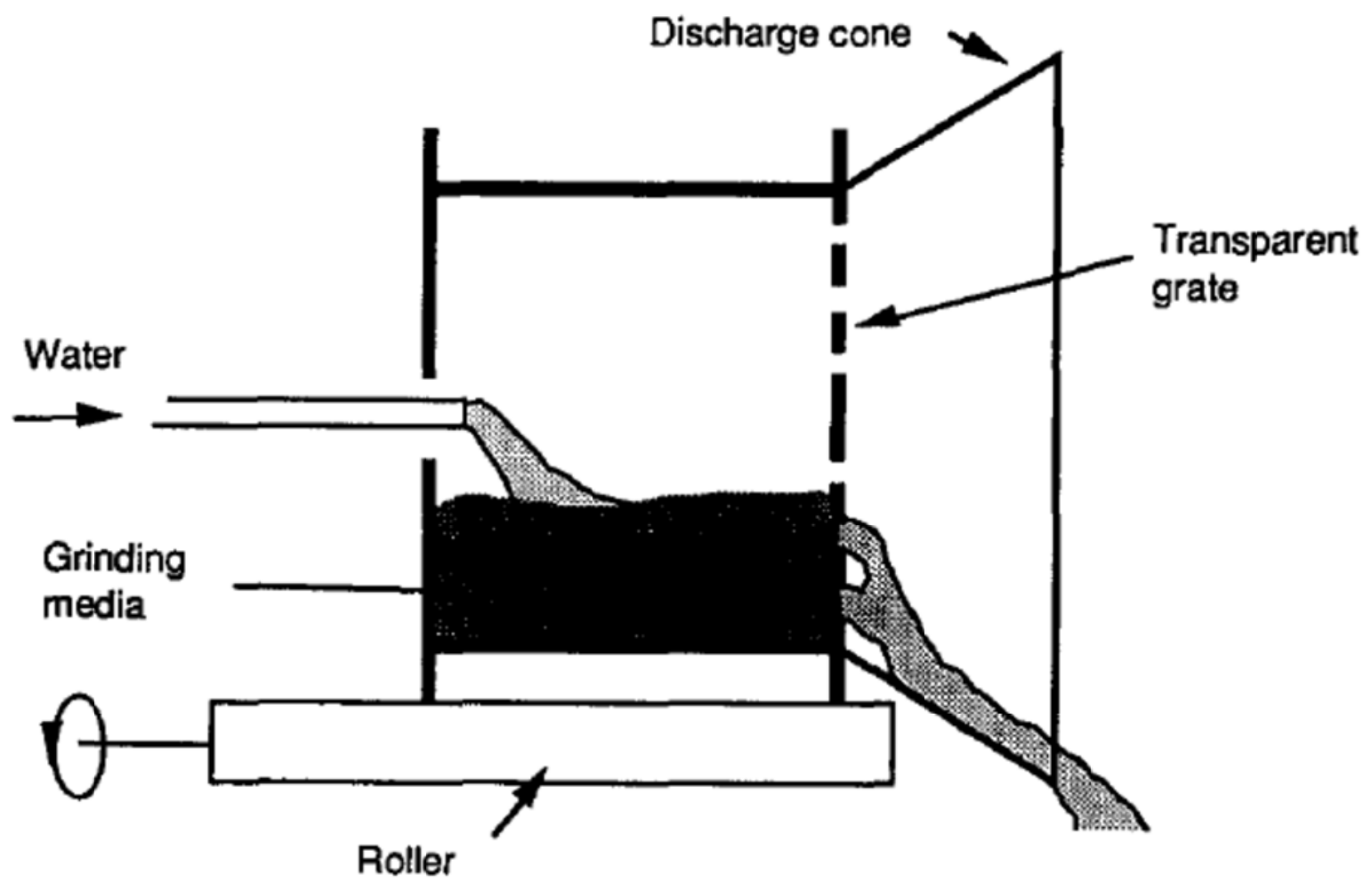

Figure 2-6 Schematic of laboratory mill used by Morrell and Stephenson (1996)

Water was fed on one end and collected via a discharge cone after passing through the grate. The grinding media comprised $6 \mathrm{~mm}$ steel balls at $30 \%$ filling fraction. In this experiment, measurements were made at steady state. When the water level would have stabilized, the water flow into the mill was stopped. Simultaneously, the flow out of the mill was collected and the volume measured. This volume constituted the hold-up. This was repeated for different grate designs, flowrates, and mill speeds. In their study, Morrell and Stephenson constructed two very important relationships. One represents flow through the charge and the other through the slurry pool. However, they considered only a narrow range of grate designs. 
Latchireddi (2002) rectified some of the deficiencies in the model of Morrell and Stephenson (1996). This was done by including a wider range of grate designs and introducing pulp lifter count. The study was done on both a laboratory and pilot scale. Latchireddi revised the original equation to come up with the following general equation:

$$
J_{s}=\eta \gamma^{n_{1}} A^{n_{2}} J_{t}^{n_{3}} \phi^{n_{4}} Q^{n_{5}} D^{n_{6}}
$$

Equation 2.3

where

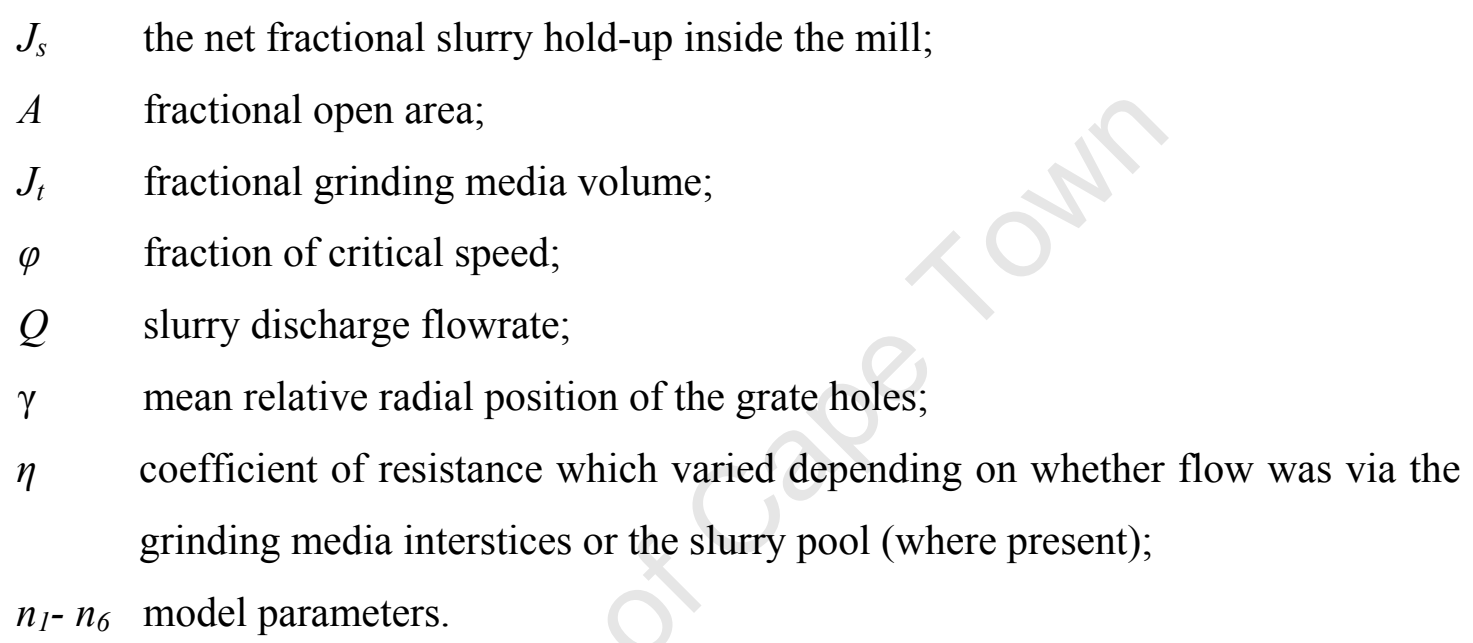

It should be noted that, while this model is very useful, it does not put into direct account the low level details of particle packing, such as porosity. Thus, the models of Latchireddi (2002) and Morrell and Stephenson (1996) can equally not be readily used for extrapolation purposes. Thus, the models are still insufficient in representing slurry transport; especially through the charge.

\subsubsection{Numerical modelling of slurry transport}

Numerical modelling has also been used in the study of slurry transport in tumbling mills. Cleary et al. (2006) attempted to model slurry transport in tumbling mills, numerically. One of the biggest challenges was the measurement of porosity. The solution was to model the charge as a dynamic DEM based porous media. The flow of slurry was modelled using smoothed particle hydrodynamics (SPH), since fluid flow cannot be modelled using DEM. Both DEM and SPH are computer simulation techniques.

The proposed sequential DEM-SPH model used in the work by Cleary et al. (2006) consists of three steps. Firstly, a DEM simulation was performed. The resulting particle data was then 
averaged onto a cylindrical grid to obtain steady state volume fraction and velocity distributions which characterise the rotating charge. The second step involved supplying the continuum porosity and velocity information, from the first step, to the SPH code. The same mill geometry was used by the two codes, though the charge representation had to be changed from discrete to continuous. Finally, an SPH simulation was performed to predict the distribution of slurry in the charge, using a supplied slurry viscosity and a Darcy law porous media drag. The latter is a function of the charge porosity and velocity distributions.

Despite the very phenomenal nature of this work, a number of issues arise. Firstly, the estimated porosity is determined by DEM, which does not include fluid flow. Cleary et al. (2006) assumed that the particulate flow is only weakly affected by the slurry. The supposition is that the slurry motion is dominated by the particulate flow of the charge. While this may be true to a great degree, it is also logical to think that the slurry does affect charge dynamics significantly, especially for high solids concentration slurries typical in tumbling mills.

Secondly, numerical models are always approximations and may be inaccurate under conditions for which equations depicting system interactions are not well established. Therefore, ignoring the effect of the fluid might lead to even greater errors. On the other hand, it is always preferable to study the real physical system rather than simulations, if one can afford it. However, the results of the work by Cleary et al. justify the non-uniformity of porosity and the need for a more accurate model of porosity. Next is an account of the effect of operating parameters on slurry transport.

\subsubsection{The effect of mill operating parameters on slurry transport}

In order to optimize mill operations, it is important to study the effect of mill operating parameters on slurry transport. A number of studies have been conducted on the relationship between slurry transport and mill operating conditions. In fact, a large part of slurry transport modelling work has been dedicated to improving operations of grate discharge mills. In this review, the focus is on the effect of operating conditions rather than design parameters.

What most studies are able to provide is the relationship between operating variables (such as mill speed) and slurry transport variables (such as hold-up). Packing properties are usually not well understood. This is because, in a 'black-box' set-up, the observer can only study changes in high level quantities such as hold-up whenever adjustments are made to an 
operating variable. Thus, researchers in this area can only speculate regarding what happens to low level properties, such as porosity, whenever mill speed is changed, for example. The focus of this section is on how slurry transport is affected by operating conditions through porosity. In particular, what changes in porosity when mill speed is changed, in order to cause a change in slurry transport? Hence, porosity is what we seek to understand, ultimately.

\subsubsection{Effect of mill speed on slurry transport}

Studies have shown that hold-up is greatly influenced by operating properties, such as mill speed. Latchireddi and Morrell (2003) studied the effect of mill speed on slurry transport. They studied a $0.3 \mathrm{~m}$ laboratory scale mill. The results were similar to those obtained by Morrell and Stephenson (1996), and Songfack and Rajamani (1999). Cleary et al. (2006) also observed a dependence of slurry flow on mill speed. One important result from the work by Latchireddi and Morrell (2003) was that hold-up increases as mill speed increases, everything else being fixed. They attributed this trend to the increase in dynamic porosity of the tumbling charge. This conclusion implies that they expected that porosity and its distribution should change with changes in mill rotation speed. They attributed the increase in hold-up, at higher mill speeds, to increased porosity as the shoulder position moves up whilst the position of the toe drops.

The reason given by Morrell and Stephenson (1996) was the changes in charge shape as speed increases. They explained that since water tends to be entrained by the charge, any change in charge shape would affect the profile of the free surface of the water and hence the flow of water out of the grate. Another observation was that, at higher speeds the free surface of the water drops in the region around the bottom of the discharge grate where most of the flow was seen to take place. In addition, they noted a reduction in the proportion of submerged open areas in the grate through which flow would take place. Figure 2-7 from Morrell and Stephenson (1996) illustrates the assertions above. 
Low speed

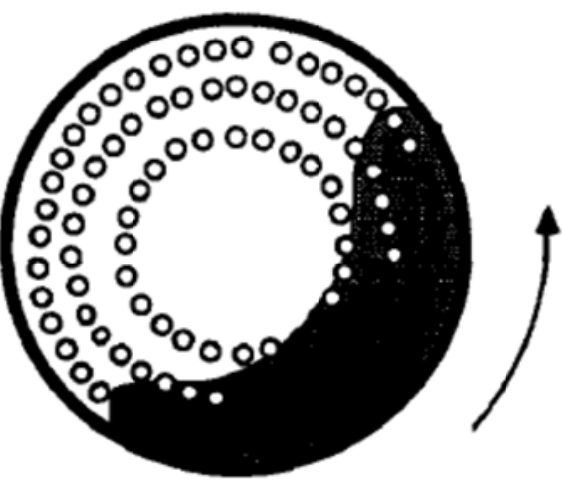

High speed

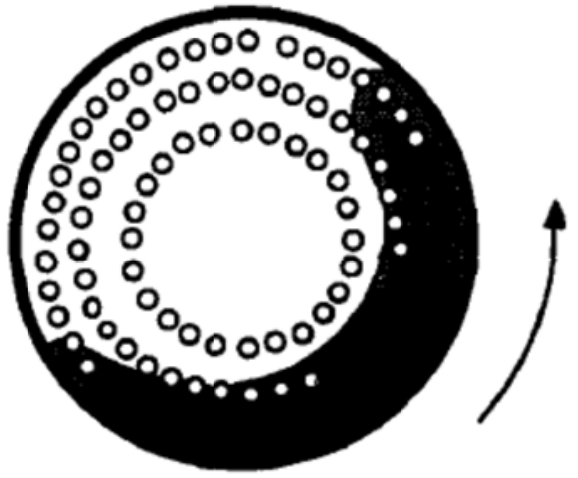

Key

Grinding media

- Apertures through which flow was observed

Figure 2-7 Schematic of observed flow out of the grate at different rotational speeds

From Figure 2-7, it is shown that, at higher rotation speeds, the charge dilates in the direction of the toe and shoulder regions. The figure also shows that the charge becomes narrower and pushes towards the mill shell at higher speeds.

\subsubsection{Effect of filling fraction on slurry transport}

Studies have also been conducted on the effect of filling fraction (or charge volume) on slurry transport. On the effect of filling fraction on hold-up, Latchireddi and Morrell (2003) found hold-up to increase with increasing filling fraction. They asserted that the magnitude of the increase was found to be dependent on grate open area. This has also been observed by Morrell and Stephenson (1996). It was noted that the onset of slurry pooling occurs at higher flowrates when higher filling fraction is considered. This is because, whereas, for the same flowrate, higher slurry levels occur in higher filling fractions, the proportion of void occupied is lower.

Latchireddi and Morrell (2003) also observed that the effect of mill speed on hold-up depends on filling fraction. This effect is minimum at high filling fractions and maximum at lower filling fractions. They attributed the dependence of the effect of mill speed on filling fraction to variations in bulk density which affects the dilation of the tumbling charge. Bulk density is a measure of the mass of a particulate system per unit volume (including void spaces). It is directly related to the packing of particles. 
It is clear from the foregoing discussions, that in order to understand the dynamics of fluid flow within the charge, charge dynamics must be adequately described. In particular, the variables that govern transport — of which porosity is key-must be understood. The effect of filling fraction and mill speed on slurry transport can only be understood fully, if there are models of spatial distribution of porosity as a function of these variables. This is because slurry transport occurs through the interstices of the charge, of which porosity is the fundamental physical descriptor. A review of fluid transport in porous media helps clarify this point.

\subsubsection{Fluid transport in porous media: Sensitivity of porosity}

In most physical processes, a link can be drawn between macroscopic and microscopic variables. In the study of fluid transport, macroscopic properties, such as pressure drop, can be linked with microscopic properties of both the porous media, e.g. pore geometry, and the fluid, e.g. viscosity. Such links can be depicted through standard models. The main focus of this section is fluid transport in unconsolidated porous media. This discussion is based mostly on the work by Evans and Civan (1994).

\subsubsection{Standard models}

Fluid flow generally involves two aspects, namely viscous force effects and inertial force effects. For low velocity flows, this can be characterised by Darcy's law (Darcy, 1856), as follows:

$$
-\frac{d P}{d x}=\left(\frac{\mu}{k}\right) v
$$

Equation 2.4

where
$P$ is pressure;
$x$ is medium distance;
$v$ is velocity;
$\mu$ is fluid viscosity;
$k$ is the Darcy coefficient.

Darcy's law is a linear relationship between pressure gradient and superficial velocity. It only includes viscous force effects. By implication, inertial effects are insignificant in slow flows. 
Forchheimer (1901) described rapid fluid flow as being governed by the following equation:

$$
-\frac{d P}{d x}=\left(\frac{\mu}{k}\right) v+\beta \rho v^{2}
$$

Equation 2.5

where

$\beta$ is the inertia resistance coefficient;

$\rho$ is fluid density.

The Forchheimer equation includes additional pressure drop observed at high velocities, incorporated by adding a term of $v^{2}$. The inertial forces depend on kinetic energy per unit volume $\rho v^{2}$. In single phase systems, the coefficient $\beta$ of $\rho v^{2}$ in Equation 2.5 is determined by pore geometry. In this case, pore geometry includes: pore size, shape and connectivity (Evans and Civan, 1994). On a higher level, pore structure is characterised by quantities such as porosity, permeability and tortuosity. Of these, porosity is the most accessible.

To accommodate fluid flow in unconsolidated porous media, Ergun (1952) developed the following general equation:

$$
\frac{\Delta P}{L}=k_{1} \frac{(1-\varepsilon)^{2}}{\varepsilon^{3}} \frac{\mu v}{d^{2}}+k_{2} \frac{(1-\varepsilon)}{\varepsilon^{3}} \frac{\rho v^{2}}{d}
$$

Equation 2.6

where:

$\triangle P$ is the pressure drop;

$L$ is length of the media;

$v$ is velocity;

$\mu$ is fluid viscosity;

$\varepsilon$ is porosity;

$d$ is particle diameter;

$\rho$ is fluid density;

$k_{1}$ and $k_{2}$ are constants. 
The Ergun equation incorporates the Kozeny theory of capillary tube model and the theory of Reynolds for resistance to fluid flow. Even though the Ergun equation does not fully represent slurry transport in dynamic beds like rotary mills, it does show that porosity $\varepsilon$ is a dominant parameter. This can easily be observed from the equation above.

\subsubsection{Porosity: a key factor of fluid transport}

Many researchers have developed correlations of the inertia resistance coefficient $\beta$, especially with permeability and porosity. By comparing the Forchheimer equation with the Ergun equation, the following correlations for unconsolidated media have been obtained (Evans and Civan 1994):

$$
\beta=\frac{0.143}{\varepsilon^{1.5} \sqrt{k}}
$$

Equation 2.7

where $k$ is permeability, and has the form:

$$
k=\frac{\varepsilon^{3} d^{2}}{(1-\varepsilon)^{2}}
$$

\section{Equation 2.8}

From the equations presented above, it can be seen that porosity is a primary factor affecting not only inertial flow effects, but also viscous force effects. Permeability is a function of porosity, pore size, shape and tortuosity. It is a key parameter in the transport of fluids in porous media and in the development of models related to this. It is however, very dependent on porosity and especially its distribution. This implies that, in order to model transport realistically, an accurate representation of porosity is required. A simple parameter sensitivity analysis also shows that porosity is a very sensitive parameter in the Ergun equation.

\subsubsection{Sensitivity analysis}

A simple sensitivity analysis was performed using the standardised regression coefficient (SRC) as the measure of sensitivity (Lawal, 2007). Given the model—with k parameters$y=f\left(x_{1}, x_{2}, \ldots, x_{i}, \ldots, x_{k}\right)$, the SRC with respect to parameter $x_{i}$ is given as: 


$$
S R C=b_{i} \frac{\sigma_{i}}{\sigma_{y}}
$$

Equation 2.9

where $b_{i}$ is the change in the output $y$ per unit increase in $x_{i}$; and $\sigma_{i}$ and $\sigma_{y}$ are the standard deviations of the input, $x_{i}$, and the output, $y$, respectively.

The table below shows results of the sensitivity analysis performed on the Ergun equation.

Table 2-1Sensitivity analysis for Ergun equation based on SRC measure

\begin{tabular}{|l|r|r|}
\hline Parameter & $\begin{array}{r}\text { Mean b_i } \\
\text { (Relative) }\end{array}$ & \multicolumn{1}{c|}{ SRC } \\
\hline Porosity & 4.498 & -1.427 \\
\hline Viscosity & 1.569 & 1 \\
\hline Velocity & 1.017 & 0.999 \\
\hline Diameter & 1.572 & -2.422 \\
\hline
\end{tabular}

Table 2-1 shows that porosity is quite a sensitive parameter. In terms of the SRC measure, the particle diameter is the most sensitive parameter, while viscosity is the least sensitive. For porosity, the SRC value of -1.427 indicates that, there is a -1.427 change in the standard deviation of the pressure drop for a unit change in the standard deviation of the porosity. The results also show that for every unit percent change in porosity, there is a 4.498 percent change in the pressure drop. These results show that porosity is a very sensitive parameter. The results are illustrated in Figure 2-8.

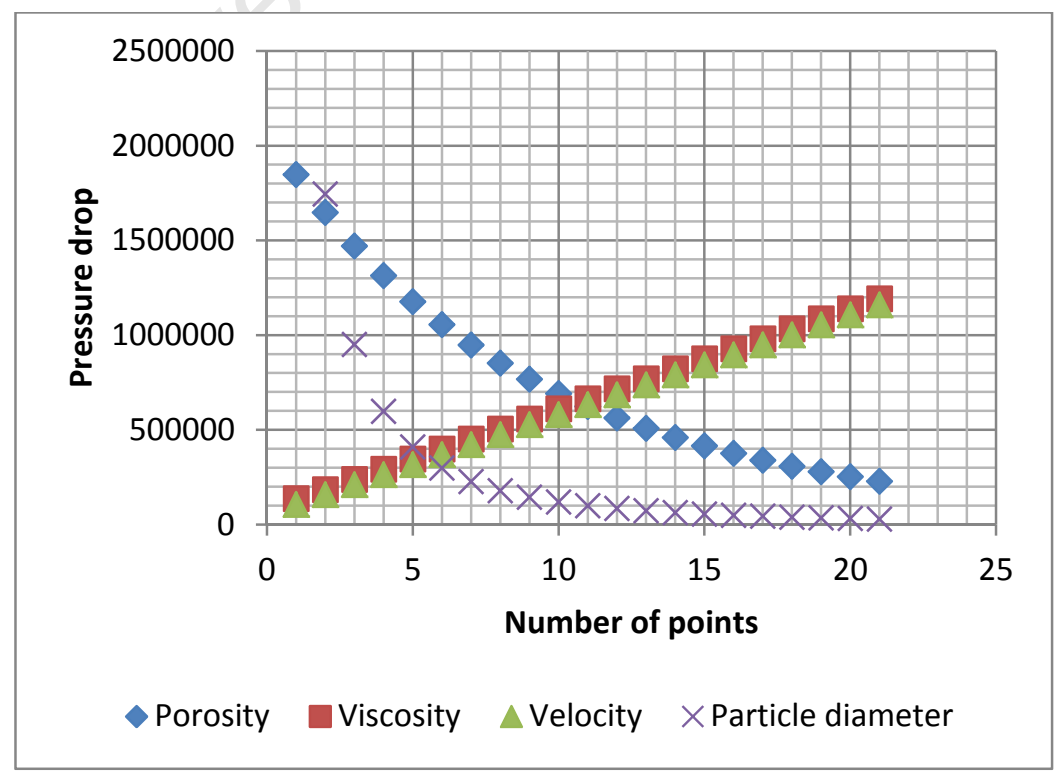

Figure 2-8 Sensitivity plots 
Figure 2-8 shows a linear correlation between pressure drop and viscosity. A similar trend is observed for fluid velocity. On the other hand, the correlations between pressure drop and porosity, and pressure drop and particle diameters are exponential.

Consequently, small errors in porosity can result in large errors in the pressure drop. It is therefore important to characterise porosity accurately, in order to effectively improve mill performance. Further rationale for mill optimisation, is provided below, through a brief discussion of energy efficiency in comminution.

\subsubsection{Energy efficiency}

Comminution by grinding is an energy intensive operation. According to a US National Materials Advisory Board (2002) report, approximately two-thirds of energy costs of mineral processing can be attributed to comminution. The report estimates energy efficiency in comminution by grinding at approximately $20 \%$. This shows that comminution is very inefficient. Therefore, optimising comminution operations and devices is vital to achieving improved mining operations. Such interventions would lead to huge energy savings. In fact, even minimal improvements would lead to significant cost and energy savings. This is due to the huge quantity of energy consumed by the mines.

Therefore, even small improvements in efficiency and performance of comminution devices are desirable. In the same vein, the sensitivity of slurry transport to uncertainty in porosity implies that small improvements in porosity characterisation would lead to improved modelling of slurry transport. This, in turn, would lead to huge gains in energy utilisation and cost reduction.

\subsubsection{Conclusion}

From the study of slurry transport in tumbling mills, it is clear that much still needs to be done; especially with respect to slurry transport through the charge. It is also evident that porosity is a dominant parameter that has also not been fully integrated into existing slurry transport models. Furthermore, it has been shown that some explanations from empirical models are insufficient due to the inadequate understanding of porosity. This adds to the need to develop a better understanding of porosity in rotary mills. Latchireddi and Morrell (2003) put it so well when they stated that:

"Estimation of [the] variation in dynamic porosity of the tumbling charge would provide the maximum slurry hold-up that can be held in grinding 
media without a slurry pool-the condition required for the best grinding efficiency".

The next section is an account of the relevant work that has been done on porosity characterisation in tumbling mills.

\subsection{Particle packing in rotating drum systems}

The question that comes from the last few sections is whether any model of porosity in rotary mills exists that could be used to study slurry transport. To the best of my knowledge, there has not been any work done on characterisation of porosity distribution in rotary mills. Therefore, most of the work in this review is based on systems similar to rotary mills - in particular, rotating drums with monosized particle beds. The systems presented here are relatively simple compared with rotary mills. However, the results of these studies provide valuable insight into particle packing in rotary mills, which are extensions of rotating drums.

\subsubsection{Porosity in rotating drums}

Porosity is one of the simplest and most accessible quantities in porous media. Porosity and packing density_one minus porosity—have been widely studied. In this review, we have also included studies where only packing density (or packing fraction) has been studied. In such cases, the simple relation between porosity and packing density is used to draw conclusions about porosity.

Porosity is a very important quantity in horizontal rotating drums. It describes the structure of the particle assembly in a very natural way. Rotating drums have wide application in many industries, including food processing, pharmaceuticals and mineral processing. Thus, many studies of porosity have been done in these areas.

It should be noted, however, that it is difficult to take direct measurements inside rotating drums. This is due to the aggressive environment within the drum, which makes it difficult to probe the rotating bed for quantities such as porosity. The only viable option then is to use non-invasive techniques. Non-invasive methods include: high-speed video and image processing tools (Santomaso et al., 2003), X-Ray (Govender et al., 2001), PEPT (Parker et al., 1993), and numerical methods such as discrete element methods (DEM). In what follows, selected studies done on particle packing in rotating drums are reviewed. 


\subsubsection{Particle flow in rotating drums}

Depending on drum rotation speed, a number of flow regimes have been observed in rotating drums. Figure 2-9 shows the different flow regimes based on DEM simulations (from Yang et al., 2008).

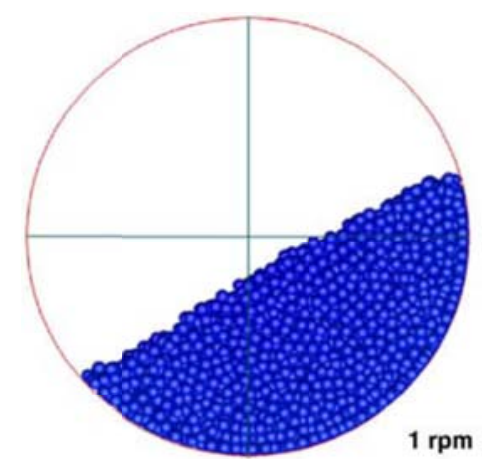

(a)

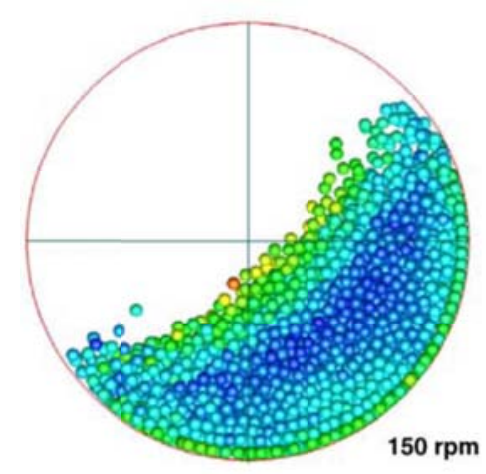

(d)

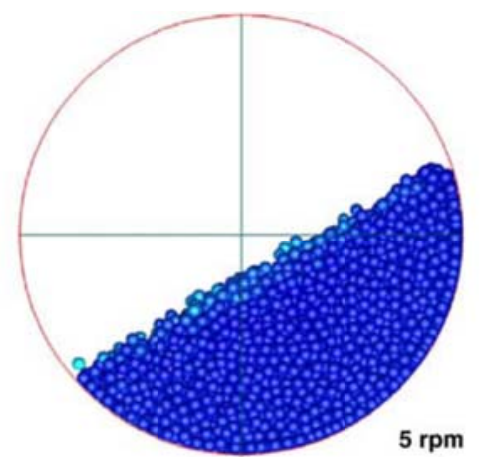

(b)

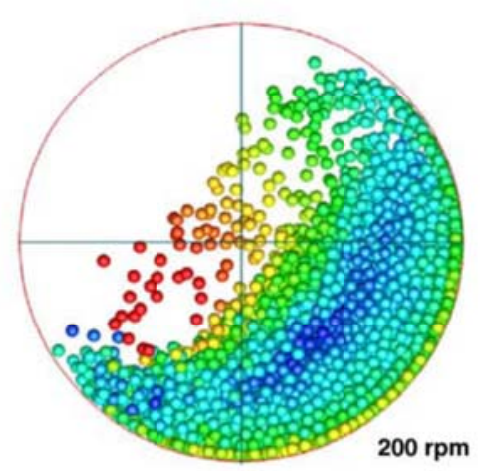

(e)

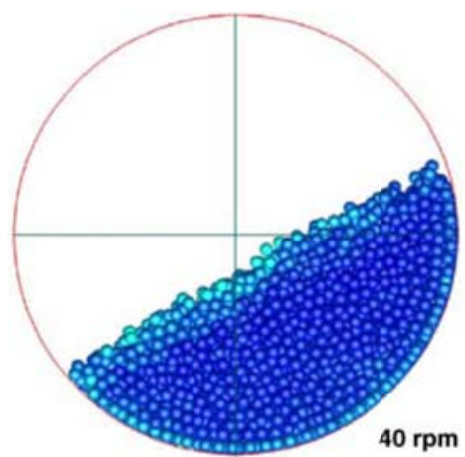

(c)

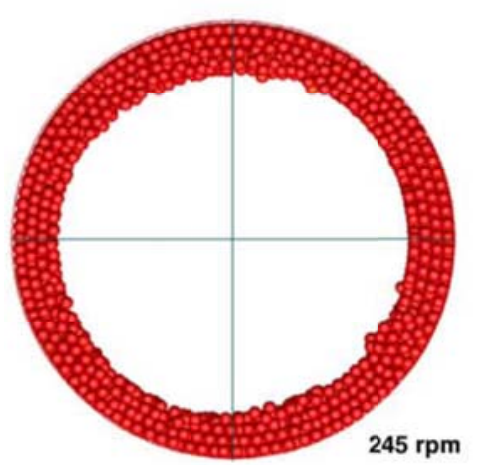

(f)

Figure 2-9 Different flow regimes represented graphically

Known flow regimes include the following (in order of increasing rotation speed): (a) slumping, (b) slipping, (c) rolling, (d) cascading, (e) cataracting and (f) centrifuging. From Figure 2-9 we see that, at different rotating speeds, the rotating bed of particles takes different shapes. The particles in the drums are also seen to behave differently with respect to speed, with particles flowing more vigorously at higher speeds. At sufficiently high speeds, particles centrifuge, as shown in Figure 2-9 (f).

\subsubsection{Spatial distribution of porosity}

The existence of a spatial distribution of porosity has been shown be many authors (Parker et al., 1997a; Yang et al., 2003, 2008; Arntz et al., 2008), both directly and indirectly. In a study on rotating drums using the PEPT technique, Parker et al. (1997a) studied particle packing 
using a single tracer particle's trajectory data. The assumption the authors made was that the packing density can be represented by the fraction of total time the tracer spends in a given region. This could only be expected if the tracer behaves in a manner representative of the whole bed. The residence time information can, therefore, help estimate the distribution of porosity across the mill. In PEPT work, this time fraction is often called the residence time fraction (Wildman et al., 2000; Wildman and Parker, 2002). Thus, the distribution of the time fractions across the mill is called the residence time distribution. Figure 2-10 shows spatial plots of residence time distribution (Parker et al. 1997a).
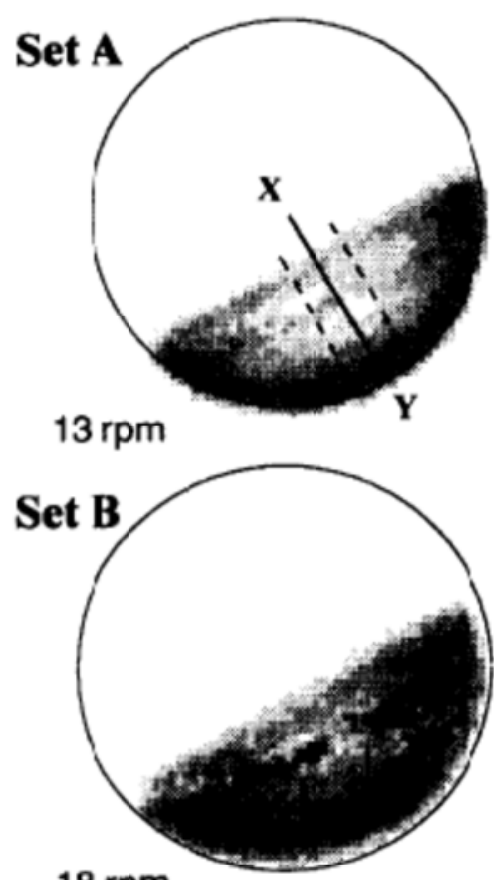

$18 \mathrm{rpm}$

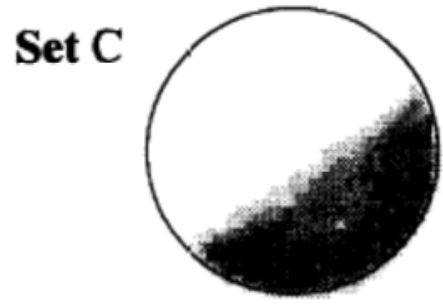

$20 \mathrm{rpm}$

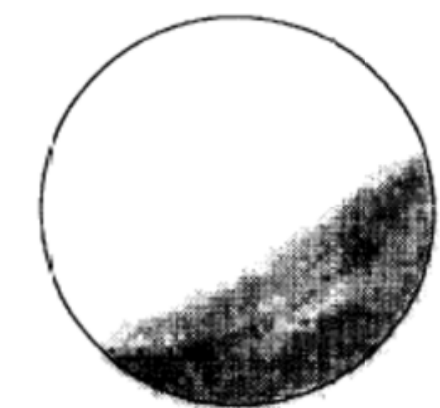

$33 \mathrm{rpm}$

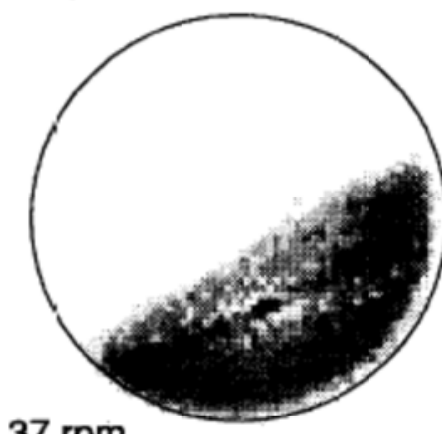

37 rpm

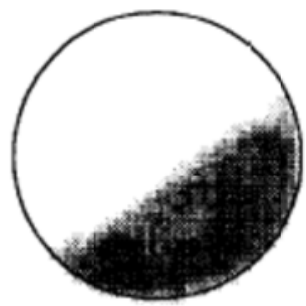

$42 \mathrm{rpm}$

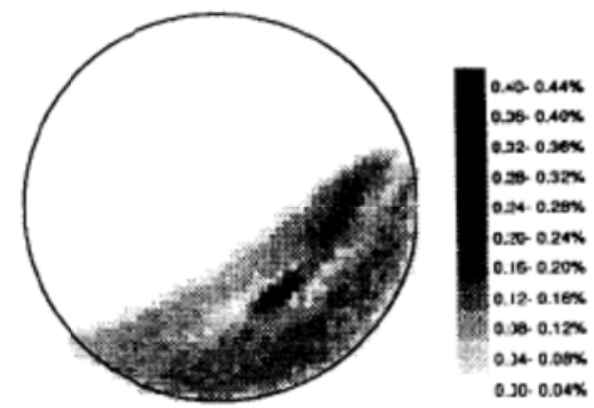

$54 \mathrm{rpm}$

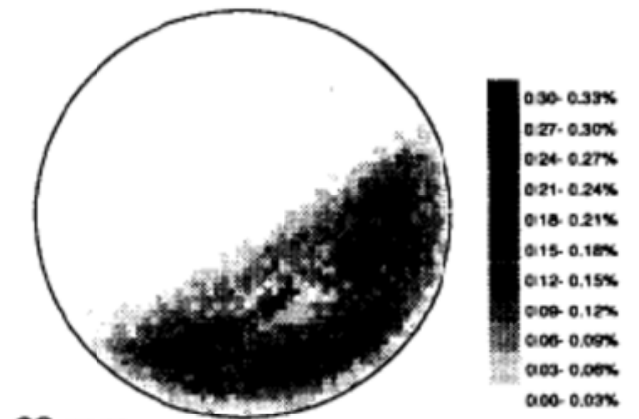

$63 \mathrm{rpm}$

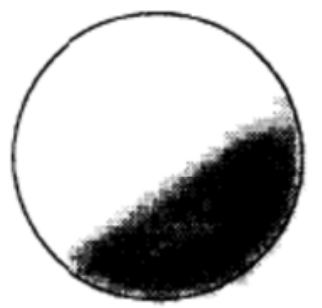

$65 \mathrm{rpm}$
$000.0 .00 x$ 0.54. 0.00x 0.46. 0.54x $0.02 .0 .20 x$ $0.36 .0 .42 \mathrm{x}$ $0.30 \cdot 0.30 \times$ $0.24-0.30 \%$ a.10. $0.26 \%$ a.12. $0.10 \mathrm{x}$ $0.0 .12 \mathrm{x}$ 0.00. 0.00\%

Figure 2-10 Residence time distributions of tracer at different configurations. Set A: $1.5 \mathrm{~mm}$ beads in $136 \mathrm{~mm}$ diameter drum; Set B: $3 \mathrm{~mm}$ beads in $144 \mathrm{~mm}$ diameter drum; and Set C: $3 \mathrm{~mm}$ beads in $100 \mathrm{~mm}$ diameter drum. 
The residence time distribution plots in Figure 2-10 give an indication of packing density across the mill, which is non-uniform. By implication, porosity is also not uniform across the mill. The plots of residence time distribution as different speeds also indicate that particle packing is affected by mill speed. Unfortunately, the range of speeds studied was very narrow and cannot give a sufficient understanding of the relationship between porosity and mill speed.

Consequently, the residence time distribution plots in the work by Parker et al. (1997a) could only be used for qualitative analytical purposes. The reason cited for this was the limitation of the tracking algorithm used. The algorithm was known to sometimes have a tendency to find the tracer at approximately the same points in a rotation cycle. However, as will be discussed later, developments in PEPT technology should help overcome these limitations. The cameras and the algorithms used currently are more accurate than previous ones. In particular, there have been major improvements in spatial resolution and increased count rate (Parker et al., 2002). Therefore, PEPT data has become more useful in quantitative studies.

In another study, Yang et al. (2003) performed microdynamic analysis of key variablesrelated to particle flow, within a rotating drum. Microdynamic analysis is a particle scale analysis of the trajectories and the transient forces of individual particles in a particulate assembly (Yang et al., 2003). DEM simulations were used to study the dynamics of a rotating drum at various speeds. The drum studied was $100 \mathrm{~mm}$ in diameter, $35 \%$ filled with $3 \mathrm{~mm}$ diameter spheres and rotating at speeds from 10 to $65 \mathrm{rpm}$. This range of speeds implies that the system was operated in rolling mode. Thus, it did not include other flow regimes. The drum also had a monosized filling.

The packing structure variables investigated were the porosity and the coordination number. Coordination number is the number of particles in contact with a given particle. The two quantities were treated as local averages, in order to establish their spatial distribution. Due to inherent fluctuations in these local quantities, time-averaging was applied to the local averages. Even though the study included a lot of aspects of particle dynamics, the focus here is on porosity. Figure 2-11 shows the spatial distribution of porosity in a rotating drum at 42 rpm (Yang et al., 2003). 


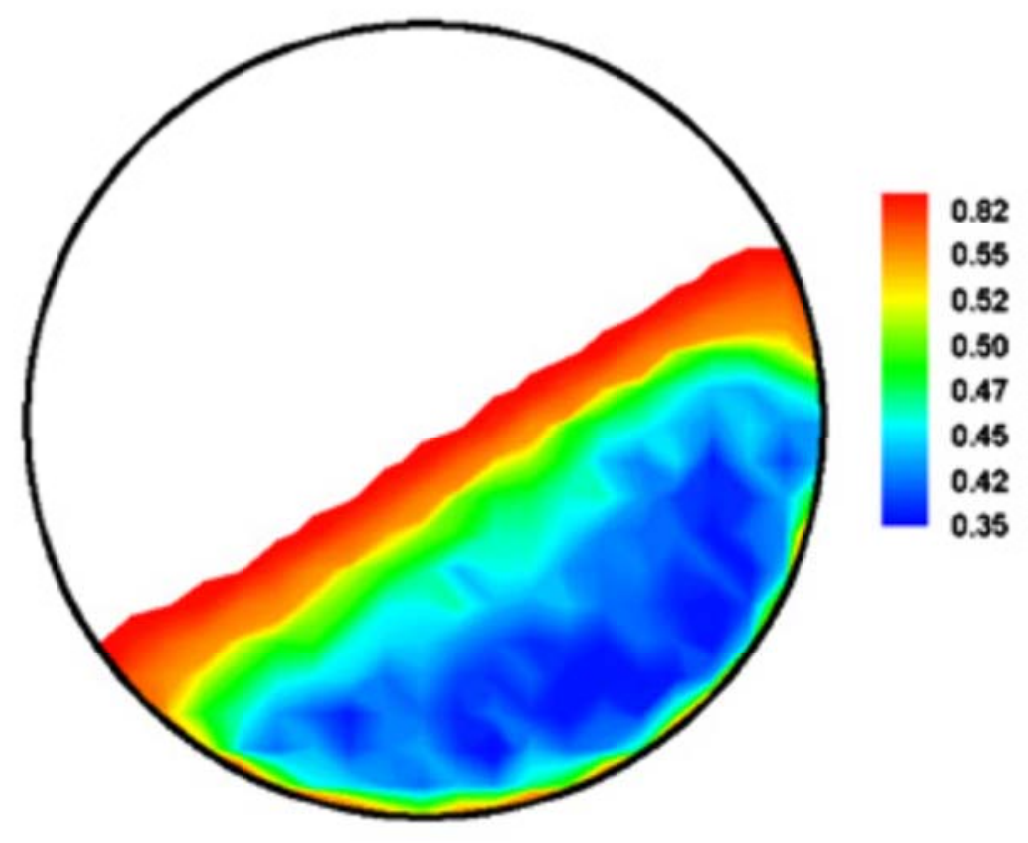

Figure 2-11 Spatial distribution of porosity

Figure 2-11 clearly shows that porosity is not uniform in a rotating drum. Yang et al. (2003) also noticed that the free surface region had a higher porosity than the rest of the bed. This was attributed to the unconfined movement of particles within the free surface. Similar spatial distributions were observed at other speeds. This should have been expected, since the range of speeds considered is within the same flow regime.

Despite being very useful, the study was limited to low speeds only, which does not depict typical rotary mill conditions. Furthermore, the drum studied did not have lifters. The contents were also monosized which is very different from the typical particle size distribution within rotary mills. However, it is clearly shown that porosity in rotary mills exhibits a spatial distribution.

\subsubsection{Correlation with operating parameters}

From the spatial plots presented in the previous sections, it is clear that the porosity distribution is dependent on drum rotation speed. Studies have been done on the correlation of particle packing with mill speed and filling fraction. Some of these are presented in this section.

\subsubsection{Effect of rotation speed}

The work by Yang et al. (2003), despite only considering one flow regime, also studied the effect of drum rotation speed on porosity. Figure 2-12 shows the results of a quantitative 
study of the variation of porosity at different speeds (20, 42 and $65 \mathrm{rpm})$ presented as probability density functions.

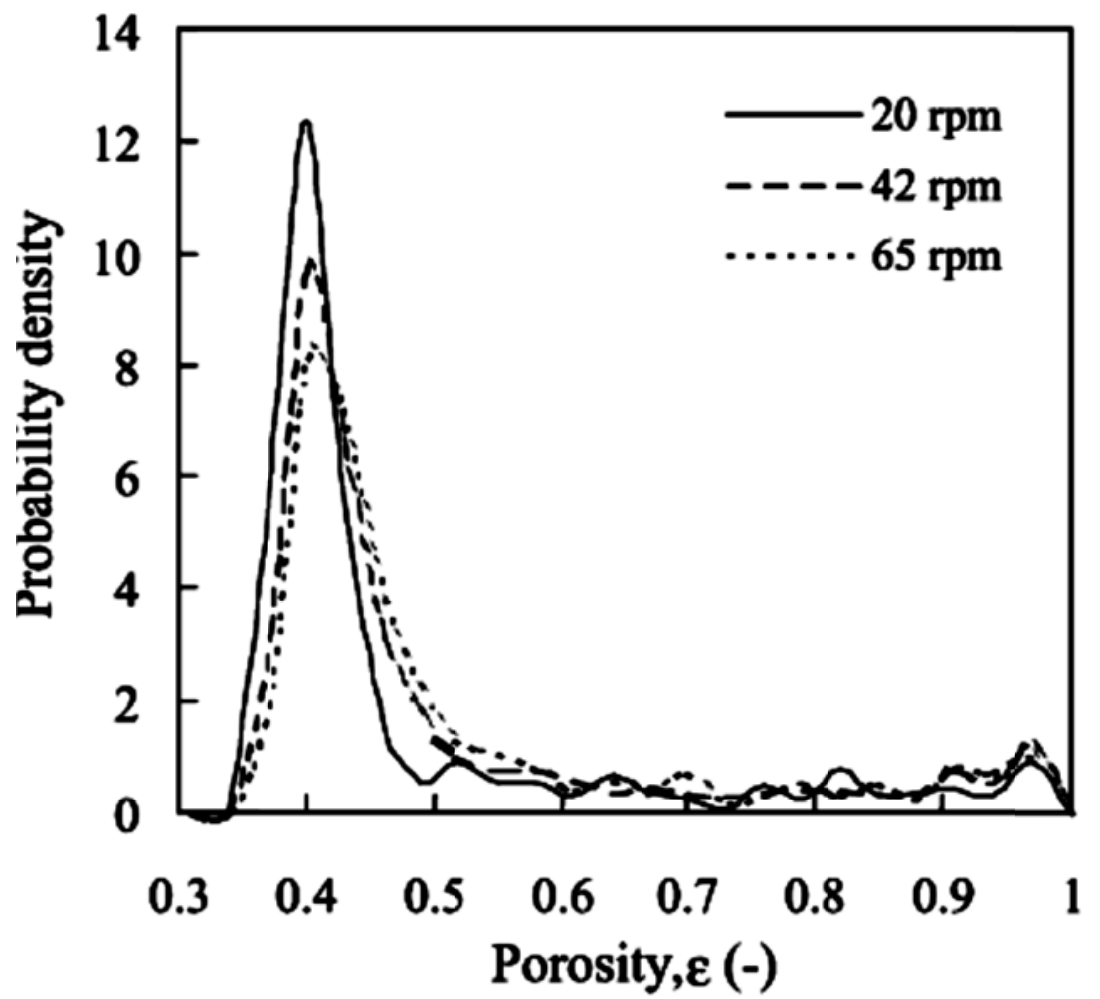

Figure 2-12 Porosity as a function of rotation speed: probability density distribution (Yang et al., 2003)

Notwithstanding the unusual use of an un-normalised probability distribution, Yang et al. (2003) observed that the probability density varies with drum rotation speed. They also observed that increasing the rotation speed decreases the peak value of the porosity and widens its statistical distribution. This, they concluded, leads to a higher overall porosity. However, they observed that although the mean porosity can be as higher as 0.6 , the peak is always about 0.4 , for the speeds considered. This observation would, therefore, imply that most of the bed is in the loose packing state. This should be expected because, in rolling mode, most of the charge is still well knit together. Furthermore, very little dilation occurs at low speeds.

Yang et al. (2003) also studied the relationship between average porosity and drum rotation speed. Figure 2-13 shows a plot of average porosity against rotation speed. 


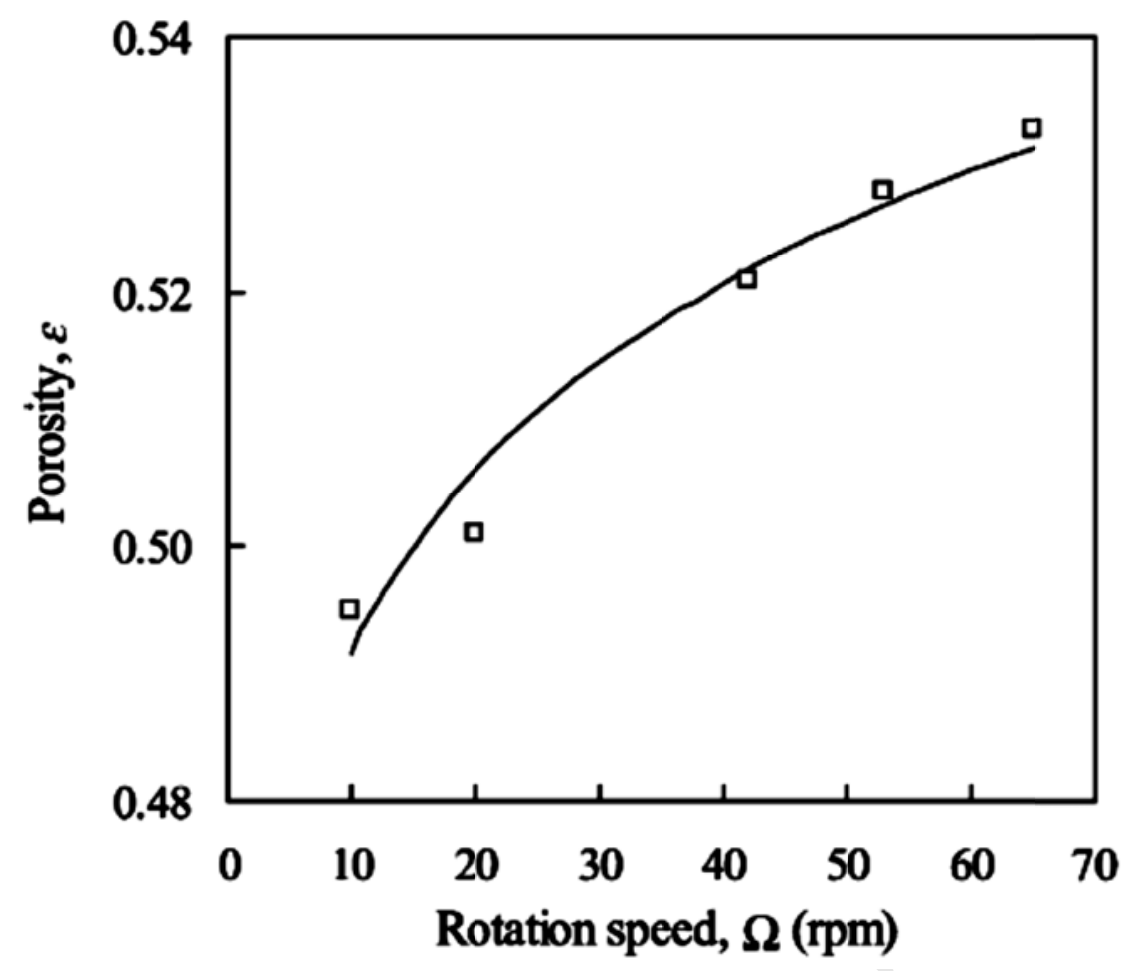

Figure 2-13 Average porosity as a function of rotation speed (Yang et al., 2003)

From Figure 2-13, it is interesting to note how much the porosity changes within the same flow regime. For speeds between 10 and $65 \mathrm{rpm}$, the porosity had a range of about 0.49 to 0.53. Since the mean porosity of the bed at $0 \mathrm{rpm}$ would be 0.40 - theoretically, it is interesting to note the sudden jump in the porosity value to approximately 0.49 at $10 \mathrm{rpm}$. This would imply that porosity should be quite sensitive to changes in rotation speed. Thus, it would be necessary to study high speeds to see how the trend evolves.

To address some issues from earlier studies, Yang et al. (2008) studied packing density for a rotating drum operated under different flow regimes. This was an extension of the earlier study (Yang et al., 2003). The simulation conditions were the same as in the previous study. The main difference was in the range of rotation speeds, which was much wider in the latter study. This was in order to investigate drum packing dynamics at various flow regimes and to quantify dependence on rotation speed. In the study, packing density was studied, as opposed to porosity. Figure 2-14 (from Yang et al., 2008) shows spatial distributions of packing density at different rotation speeds (and flow regimes). 

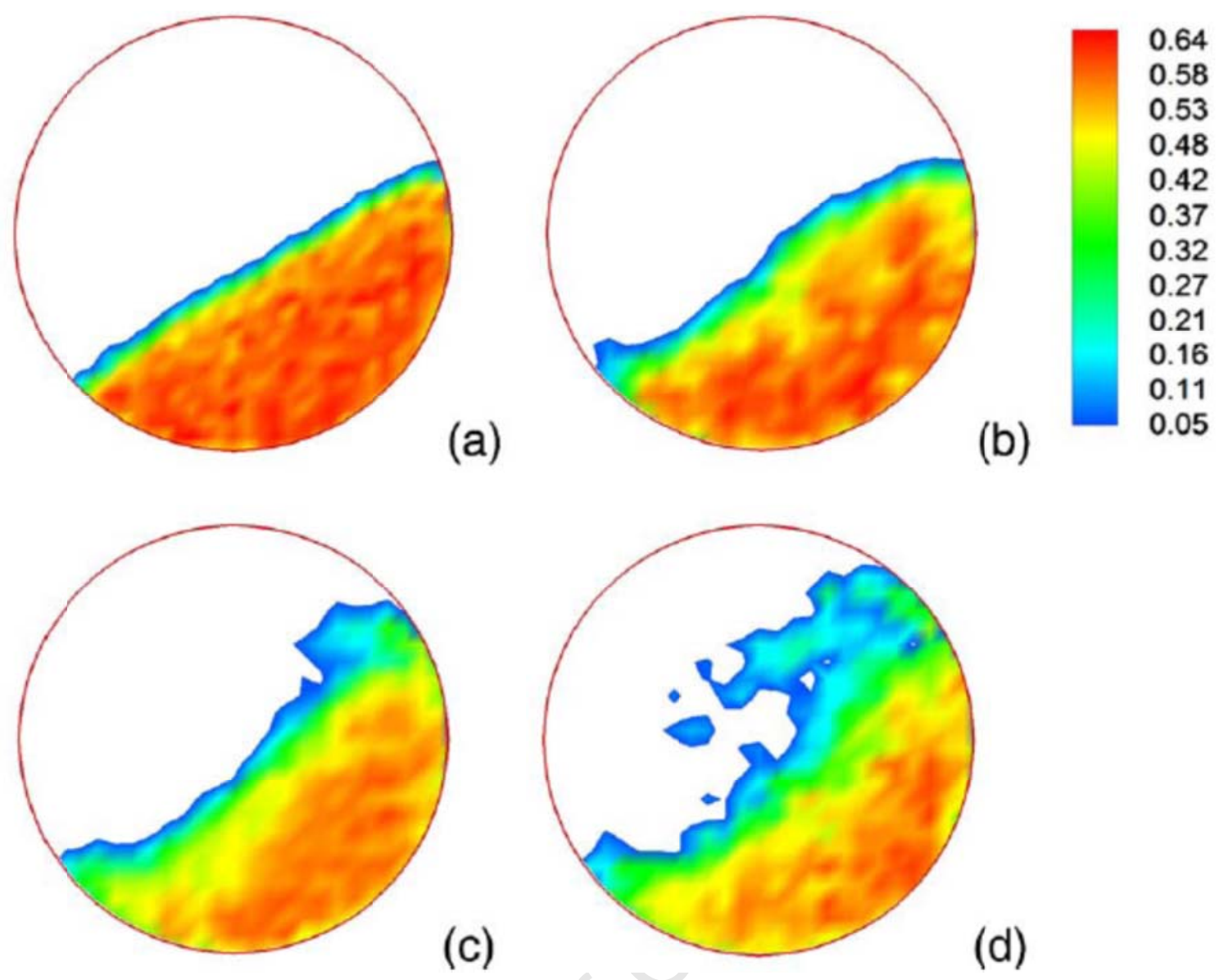

Figure 2-14 Spatial distribution of porosity at different speeds: a) 5rpm; b) 80rpm; c) 150rpm; d) 200rpm

In the diagram above, the hot colours represent high packing density (or low porosity). Yang et al. (2008) observed (in Figure 2-14 (a)) that at 5rpm (rolling regime), which is lower than all the speeds in their previous study, the packing is non-uniform. The solid bed has a higher packing density while the flow surface has a much lower density. However, the packing density is more evenly distributed.

They also noted that, as rotation speed increases, the bed expands in volume or dilates. This implies that the mean packing density decreases - while the mean porosity increases. There is, therefore, a correlation between mean porosity and rotation speeds for different flow regimes. Yang et al. (2008) also observed that, at higher speeds, the shape of the bed also changes. The shape of the surface in rolling mode can be seen to be relatively flat. At higher speeds, an S-shaped free surface is evident. This has also been observed by other authors (Santomaso et al., 2003; Taberlet et al., 2006). It should be expected that the change of shape implies a change in the porosity distribution. 
Other phenomena as speeds increases include an increase in the number of particles cataracting (in free flight). There is also an increase in the number of particles flowing within the free surface (Yang et al., 2008). Visually, a lower packing (or higher porosity) across the drum is evident. This deduction is supported by the higher average porosity observed at higher speeds (see Figure 2-16). We can also see that the non-uniformity of packing density increases. This implies that the porosity values also vary more significantly and that its nonuniformity across the drum is more pronounced at higher speeds. Figure 2-15 shows probability density plots for four different speeds (5 rpm, $80 \mathrm{rpm}, 150 \mathrm{rpm}$ and $200 \mathrm{rpm})$, which confirm the foregoing observation.

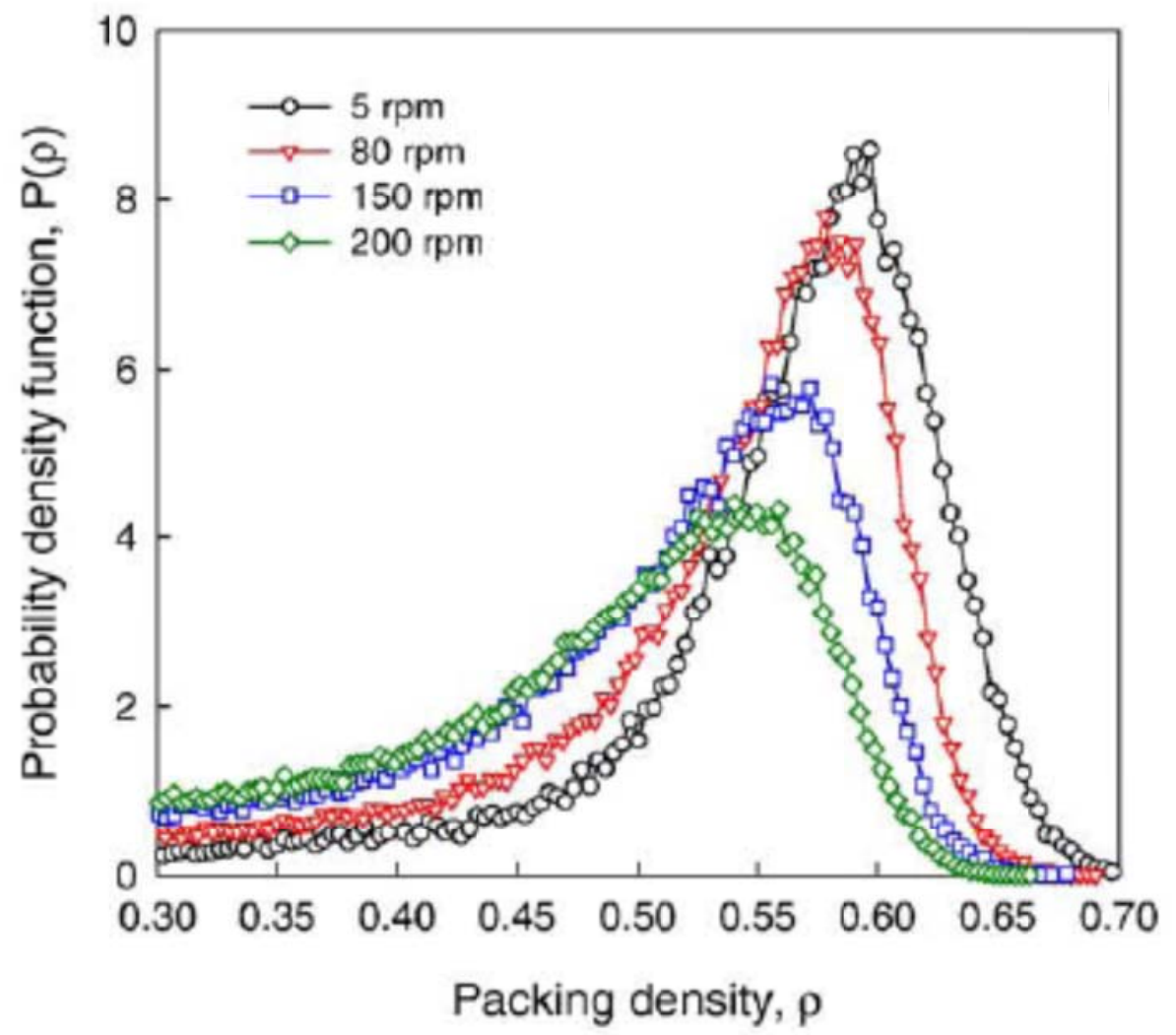

Figure 2-15 Distributions of packing density at different speeds (Yang et al., 2008)

Figure 2-15 shows the statistical distribution of packing densities at different rotation speeds. Yang et al. (2008) observed that all distributions show strong peaks. It was also observed that with increasing speed (due to dilation of the rotating bed), the peak value of packing density decreases and the distribution widens. This can be observed in the lower peak and wider distribution at $200 \mathrm{rpm}$, compared with other curves. It is this variation in packing density 
distribution that is given as a reason for the observed lower packing density at higher speeds. Figure 2-16 shows the relationship between mean packing density and rotation speed.

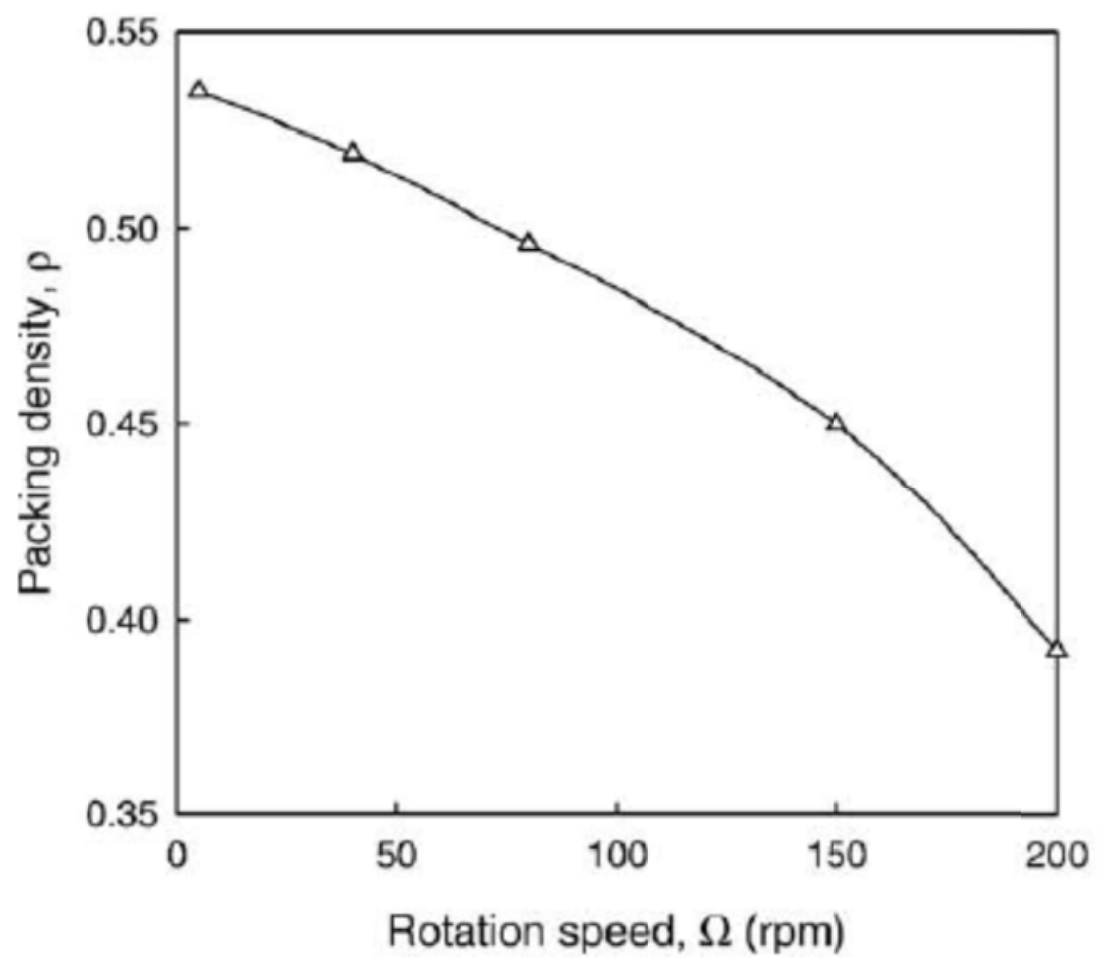

Figure 2-16 Mean packing density at different rotation speeds (Yang et al., 2008)

From Figure 2-16, it is shown that packing density decreases with increasing rotation speeds. The range of packing density was approximately 0.38 to 0.53 . On the other hand, porosity increases with increasing speed and would range from 0.47 to 0.62 .

Based on the study by Yang et al. (2008), the strong peaks observed in Figure 2-15 would also be observed had the statistical distribution been based on porosity. Furthermore, due to bed dilation at higher rotation speeds, the peak value would also decrease and the distribution would be wider.

Dilation has been observed by many authors (Ding et al., 2001, 2002; Arntz et al., 2008). Ding et al. (2001) studied solids motion in a rotating drum and noted that the bed material is dilated at high speeds. They also observed that dilation increases with increasing rotational speed, estimating it greater than $10 \%$ at high rotation speeds. When the charge dilates it has higher average porosity. Dilation also implies that porosity distribution varies with mill speed. 
Using results from studies of the simple systems presented above, we can begin to explain some of the observations made in empirical slurry transport modelling. For example, the reason there is increased hold-up at higher speeds can be attributed to increased porosity due to charge dilation. The results above also confirm some of the assertions regarding porosity made by researchers involved in modelling slurry transport.

\subsubsection{The effect of filling fraction}

The filling fraction, as earlier observed, is an important factor affecting slurry transport through the mill charge. A number of studies have been conducted on the effect of filling fraction on particle packing in rotary mills and similar systems (Santomaso et al., 2003, Arntz et al., 2008). Santomaso et al. (2003) study packing density in a rotating drum with inner diameter of approximate $390 \mathrm{~mm}$ and about $600 \mathrm{~mm}$ long. The drum was filled with glass beads, at three different filling fractions: 10,15 and $25 \%$ by volume. Figure $2-17$ shows the outlines of the free surface at the three different filling fractions.

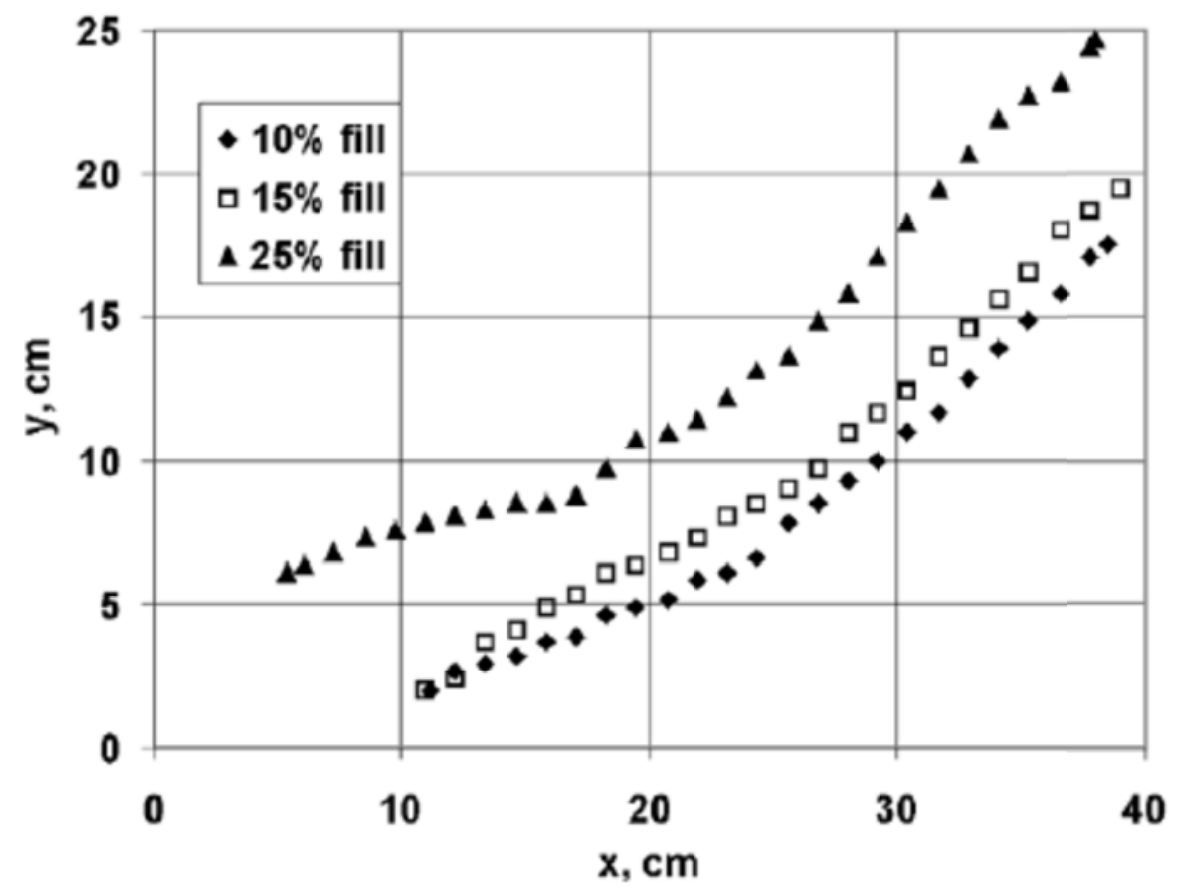

Figure 2-17 Effect of drum fill on the surface shape of the particle bed (Santomaso et al. 2003)

In Figure 2-17, the profile of the free surface is shown to vary with filling fraction. The first and the last data points in each series represent the locations where the bed surface contacts the drum wall. Even though the shape of the functions shown above may not be significantly different, changing filling fraction can greatly affect packing characteristics, including segregation (Rapaport, 2007). For example, Rapaport (2007) observed that sufficiently high 
filling fractions favour permanent radial segregation, while at the same time suppressing axial segregation. Thus, the relationship between porosity and filling fraction is an important subject in particle packing studies.

One important issue not adequately addressed by previous studies on porosity in rotating drums is the particle size distribution of the bed inside the drum. It is important to study such systems if porosity in rotary mills is to be adequately characterised. This is because the packing of mixtures is very different from that of monosized systems (German, 1989). In fact, according to Liu and Ha (2002), particle size distribution is probably the most significant variable affecting packing of particles. What follows is a summary of some of the work done in this area.

\subsubsection{Porosity of multi-component mixtures in rotating drums}

The porosity of multi-component mixtures has been studied by many authors (Ouchiyama and Tanaka 1986, Standish and Yu 1987; Yu and Standish 1987, 1991; Dias, et al. 2004). Most of this work involved the formulation of mathematical models for predicting the porosity of mixtures. For example, the linear-mixture packing model and its variants have been widely used in predicting the packing of mixtures. These models involved the use of each component's particle size and the volumetric ratios of these components. The systems in this case are stationary. The studies above have shown that the packing of monosized particles is different from that of multi-component mixtures. This is due to the fact that in particle mixtures, smaller particles tend to fill the interstices that may occur among larger particles (Dias et al. 2004). However, such mathematical models are difficult to apply to aggressive systems like rotating drums, since they were developed for simpler systems. In fact, for dynamic systems, appropriate corrector functions are necessary in order to arrive at accurate porosity estimates (Dias et al., 2004).

For rotating drums, most of the work done to date has been on monosized systems, as discussed in earlier sections (Yang et al., 2003, 2008). A significant amount of work has also been done on binary systems (Arntz et al., 2008) for the purpose of studying phenomena such as size segregation and mixing (Baumann et al., 1994; Dury and Ristow, 1999). Some work has also been done on packing density. However, to the best of my knowledge, no work has been done on the porosity of multi-components in rotating drums. On the other hand, binary systems present a bridge between monosized and multi-component systems. Thus, they can provide useful insight into the porosity of higher order mixtures and how porosity can be 
measured in multi-component mixtures. Arntz et al. (2008) studied the packing density of a binary system at different speeds. Figure 2-18 illustrates this result for a binary mixture with sizes 2 and $3 \mathrm{~mm}$ in radius, in a rotating drum at 50\% filling fraction. It shows the packing density at four different mill speeds.
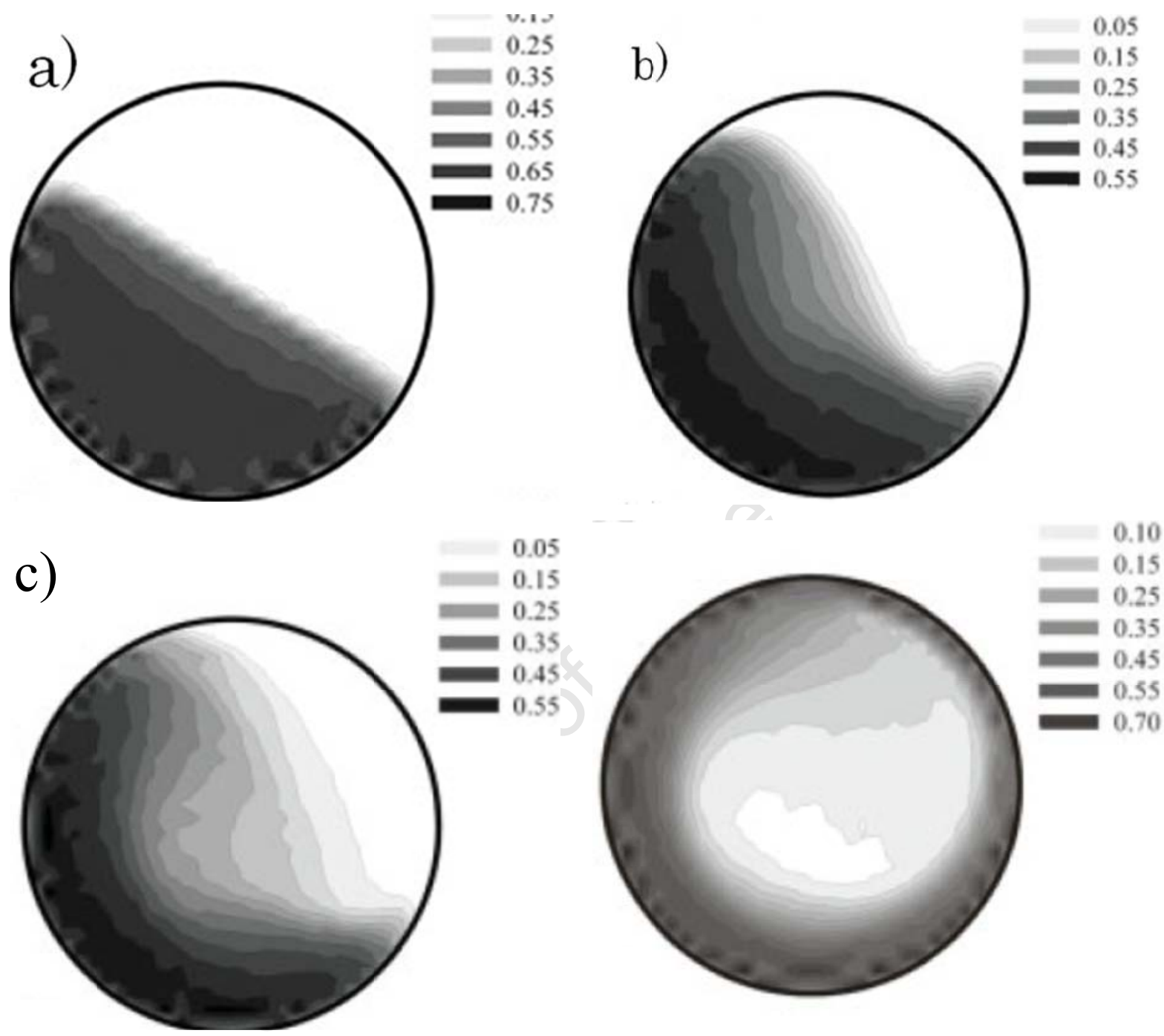

Figure 2-18 Analysis of the bed properties for mill speeds in: a) the rolling regime (1.57 rad/s); b) the cascading regime $(12.56 \mathrm{rad} / \mathrm{s}) ; \mathrm{c})$ cataracting regime $(15 \mathrm{rad} / \mathrm{s})$ and; d) cataracting-centrifuging regime (19rad/s) $($ Arntz et al., 2008)

From Figure 2-18 the existence of a spatial distribution is very clear. Arntz et al. (2008) showed that the particle bed expands at higher speeds during mixing and segregation. For instance, the volume of the charge in the cascading regime is higher than that of the rolling regime. We can also see that there is a difference in bed shape. Notice also that in Figure 2-18 (b) and (c) the bed expands towards the shoulder and toe. The authors also observed variations in packing density from the free surface to the mill shell. In particular, there is a 
lower packing in the free surface region compared with the passive bulk. The variations are shown by the contours in the plots.

There is also a band of higher packing (lower porosity) towards the shell, which forms at higher mill speeds. This can be seen in the cascading and cataracting regime. This was not observed in the work by Yang et al. (2008), where the band of lower porosity is not distinguishable. This indicates strong possibility that porosity in rotating drums does depend on particle size distribution. For the cataracting-centrifuging regime, the particle bed is spread out along the mill shell, with a more uniform but lower packing. For more than two components, the porosity distribution is expected to be even more different, and more difficult to predict (Dias et al., 2004). The porosity of multi-components is, therefore, worth investigating.

On the other hand, the work by Santomaso et al. (2003) studied three different sizes of particles, but only separately.

\subsection{Conclusion}

There are two major issues that have been identified in this chapter. The first is that the assumption made when formulating many existing models of slurry transport, that charge porosity is uniform, is inadequate. It is very clear from the literature that charge porosity in rotary mills is not uniform. This has been demonstrated through spatial distribution and probability density distribution plots of porosity. Furthermore, studies of fluid transport in porous media show that porosity is a dominant parameter in modelling fluid transport in porous media. It is, therefore, important to develop an accurate model of porosity in rotary mills.

Secondly, despite the useful work on the descriptions of porosity in dynamic systems such as rotary mills, a number of issues have not been fully addressed. To start with, most of the studies reviewed are based on much simpler systems, compared with rotary mills. For example, the systems have no lifters which are a key feature in rotary mills. With regards to lifters, Santomaso et al. (2003) proposed future study on systems that include them. In fact, some studies have shown that lifter design affects particle packing (Djordjevic et al., 2004). Other major issues include inadequate treatment of particle size distribution, mill rotation speed and the filling fraction. 
It should also be noted that, in studies where the foregoing issues have been considered, only one or two of them have been included together in a single study. Examples are the work by Yang et al. (2008) and Santomaso et al. (2003). In particular, the work by Yang et al. considered a range of mills speeds in their investigations. However, the charge was monosized and the drum had no lifters. Other work, Arntz et al. (2008) studied the effect of both filling fractions and mill speed on porosity. However, the particle bed was a binary mixture and the mill had no lifters.

Based on the foregoing discussions, knowledge gaps still exist in porosity characterisation in rotary mills. This is not to say there is no valuable information to get from studies on rotating drums, presented above. However, in order to achieve the goal of mechanistic slurry transport modelling in tumbling mills, there is a need to study systems which adequately represent realistic milling conditions. The widest of the knowledge gaps has to do with the study of multi-component mixtures in rotary mills. In addition to these issues, other studies that might have been done on systems more like rotary mills do not characterise the porosity of the charge in detail. This is, perhaps, because porosity and packing density were not the primary subjects of these studies.

The aim of this thesis has been to develop better characterisation of charge porosity in rotary mills. To help understand tumbling mill charge porosity, it was important to consider studying the various aspects of tumbling mills together. This would help characterise porosity in the mill under realistic conditions. To undertake this work, it was important to decide on the research approach and methodology. This is discussed in the next chapter. 


\section{Research objectives and approach}

\subsection{Research strategy}

The approach followed in this work was to characterise porosity distributions based on fundamental principles. The first problem, therefore, was to find ways to collect particle level data, such as particle position, within the mill. Thereafter, techniques for computing porosity using this data were developed. In addition, the best way to represent porosity distributions had to be decided upon. The final step was to model porosity as a function of mill operating conditions: mill speed and filling fraction.

\subsection{Data collection methods}

Deciding on data collection techniques, as well as developing numerical schemes for computing porosity, required a study of the methods employed in the literature. There are numerous ways to measure the porosity of particle systems. However, this section discusses only a small number of these techniques-those most relevant to this work. What is needed is a method that could help overcome the various challenges that are faced in the study of porosity in rotary mills.

\subsubsection{Direct measurements}

The simplest way to measure porosity is to fill the voids within a porous bed having a bulk volume $V_{b}$ with a liquid. Thereafter, the volume of liquid $V_{w}$ that saturates the bed can be used to compute porosity, as follows:

$$
\varepsilon=\frac{V_{w}}{V_{b}}
$$

Equation 3.1

This method can be used quite effectively with a stationary system in a container. However, it cannot give an indication of the porosity distribution. In addition, due to the aggressive nature of the tumbling bed in a rotary mill, it is impossible to take direct measurements.

It is such difficulties that have led to extensive work on indirect measurement methods. These include computer simulations and non-invasive experimental techniques. Computer simulation methods used in the study of particle dynamics include discrete element methods 
(DEM) and Monte-Carlo simulations. Non-invasive experimental techniques include: positron emission tomography (PET), X-Ray imaging and the positron emission particle tracking (PEPT).

\subsubsection{Discrete element method}

Of the various numerical methods employed in the literature, DEM has been the most widely used in approximating the physics of particulate systems involving discrete particle interactions - typical of tumbling mills. DEM involves following the motion of individual particles in a granular flow system. It models the interaction between neighbouring particles and their containers based on certain boundary conditions. In DEM, forces acting on each particle are approximated by applying fundamental Newtonian mechanics principles via a chosen contact model. Using the net forces acting on each particle from such interactions, the particle's acceleration is computed using its (known) mass. By numerical integration of acceleration, velocity is approximated. Displacement is then calculated by second integration of acceleration. By keeping track of the position of each particle, a system of particles can be simulated quite accurately.

DEM has been widely used in tumbling mill research (Mishra, 2003), since its introduction (Cundall and Strack, 1979). However, it does not include fluid flow through the particle assembly. Due to this, and the inherent inaccuracies that come with simplified contact models and boundary conditions, there is growing interest in the development of techniques that can be used to make equivalent measurements in real systems. Moreover, all models are approximations. Thus, studying real systems would also help augment numerical modelling efforts by testing their validity in the scenarios of interest.

\subsubsection{Positron emission particle tracking}

A strong alternative to computer simulations is non-invasive particle tracking methods, which have been used in studying similar systems (Parker et al. 1997a; Govender et al., 2001). Of these methods, PEPT has emerged as a very powerful and useful tool. This method has found wide application in many studies of dynamic systems, including rotating drums (Parker et al., 1997a). In particular, it has been employed as a measuring technique in the packing of both monosized and binary systems (Wildman et al., 2000; Wildman and Parker, 2002). In PEPT, the motion of a single radioactively labelled particle is tracked for a considerable period. Thus, this method can capture in-situ particle position data as a function of time. Therefore, the dynamics of real aggressive system can be studied without interfering with the system. 
Despite only tracking one particle, following the particle's trajectory for a considerable period results in the accumulation of pseudo whole system information, at the particle level. Such information can then be used in estimating system microdynamics which are useful in optimising design and operational parameters. The biggest problem is that there is only limited information generated from tracking one particle. Therefore, to make good use of the data, it is important to develop robust techniques for analysing the trajectory data. This poses a number of challenges, some of which have already been addressed.

\subsubsection{Data collection method}

In this work, data was collected using PEPT. What is most attractive about this technique is that a real system can be studied. Important questions that had to be asked include the following:

- Do techniques exist that can be used to compute porosity in rotating drums using PEPT?

- If so, can we adopt some of the techniques in the current problem?

- If not, can we improve upon these techniques, or do we need to develop new ones?

Fortunately, methods for characterising the packing of monosized systems already exist and will be discussed shortly. However, with regards to multiple component mixtures, no such methods exist - to the best of my knowledge. Despite the existence of studies of binary mixtures using PEPT (Wildman and Parker, 2002), only the packing of individual size components has been reported. The assumption in these studies has been that, if different tracers are tracked - in turn - each tracer represents particles similar to it (Wildman and Parker, 2002; Parker and Fan, 2008). Thus, it is possible to study the packing patterns of individual components if different sized tracers are used, in turn.

In this thesis, the aim was to study the porosity of the multi-component mixture as a whole. Therefore, one of the major challenges was to develop techniques for computing porosity in such systems. In other words, it was essential to develop techniques for combining the results of studying individual components via the different tracers' trajectory data.

\subsubsection{Experimental design considerations}

The development of the techniques has a huge bearing on the experimental program. This is because, for experimental data to be meaningful, it is important to consider the how the data 
would be used during experimental design. As a result, an iterative approach was followed in this work. The first set of experiments was performed before fully developing the techniques. Upon fully developing the techniques, another set of experiments was performed to test the model for limitations. However, with regards to the tracers, different sized particles should be tracked, in turn, with the experiment (same configuration) repeated as many times as the number of size classes in the system. This is in order to reflect the behaviour of the whole system.

\subsection{Development of techniques}

The techniques for computing the porosity of multi-component mixtures are based on fundamental principles from mathematics (ergodic theory) and physics (statistical mechanics). Important assumptions are made in the process. These are based on the ergodic hypothesis. The ergodic hypothesis asserts that time averages are equal to space averages of the ensemble. This is what provides for the use of a single particle's trajectory data as a means to compute physical quantities relating to the whole system. The result of the development of techniques was a set of mathematic formulae. These have been converted into computer programs in MATLAB and used to generate useful results presented in the results section of the thesis.

\subsection{Research design}

\subsubsection{Key questions}

Based on the thesis goal, the background and the methodological considerations presented above, the following are the key research questions:

- Can single particle tracking data be employed in computing spatial distributions of porosity in a multi-component mixture within a rotary mill?

- How is porosity distributed radially from the charge free surface?

- How does porosity distribution vary with mill speed and filling fraction?

\subsubsection{Hypotheses}

The following hypotheses were then formulated base on the key questions and theory: 
- The time-averaged packing density provided by a tracer equals the equilibrium average packing density of its size class, because packing density is invariant at steady state and a particle tracer represents other particles from its size class.

- The porosity of any given volume element in the charge at steady state is a linear combination of solid volume contributions of individual size components, due to coexistence of the individual components within each volume element.

- Porosity decreases with increasing radius from the free surface of the charge, due to an associated increase in particle number density towards the mill shell.

- Porosity increases with increases in mill speed due to charge dilation, which results in a reduction in packing density, on average.

\subsubsection{Objectives}

To test the hypotheses given above, the following are the research objectives:

- Develop mathematical and numerical frameworks for calculating (from PEPT trajectory fields):

- Solid volume contribution of each size component in a given volume element;

- Porosity distributions through the combination of the various size components based on the solid volume distributions computed above;

- Study the variation of porosity distribution with respect to:

- Radial position from the free surface across the relevant region of the mill;

- Mill speed;

- Filling fraction.

\subsection{Conclusion}

The research strategy employed here involves development of mathematical formulations, development of computer programs, experimental work and modelling. These aspects form a large part of the rest of this thesis. The next chapter discusses the PEPT method, in view of its application to the current study. It forms the basis for the remaining chapters where the mathematical formulations, the experimental setup and results are presented. 


\section{Positron Emission Particle Tracking}

The positron emission particle tracking (PEPT) is a measurement technique developed at the University of Birmingham in the United Kingdom (Parker et al., 1993). It is a variant of the positron emission tomography (PET) that is commonly used in studying metabolic process in medical diagnostics. PEPT was designed to be used in the study of engineering processes using PET technology. Consequently, it has found wide application in the study of dynamic systems in engineering and science. For instance, it has been applied to the study of particulate systems and flow in multiphase systems (Parker et al., 1997b; Cox et al., 2003; Bakalis et al., 2006; Depypere et al., 2009). What follows is a detailed account of the PEPT technique. The focus is on how PEPT is, or can be, used in the measurement of porosity in rotary mills and related systems.

\subsection{The technology}

PEPT entails the tracking of a radioactively labelled particle, called a tracer, inside a dynamic system. The tracer particle is labelled using a radionuclide which undergoes $\beta$ decay and emits positrons. The positron annihilates with an electron to produce a pair of essentially back-to-back $511 \mathrm{keV} \gamma$-rays, termed the line of response (LOR). These $\gamma$-rays are very penetrating and can be captured by special positron cameras, for further processing. Therefore, in order to follow the tracer's trajectory, the system being studied is placed between detectors (forming the positron camera). It is the penetrating power of $\gamma$-rays that enables the PEPT method to be used for studying opaque systems, making it a powerful noninvasive technique for studying particle dynamics. The next few sections discuss aspects of tracer-labelling, the cameras used and how tracer position is determined.

\subsubsection{Tracer labelling}

There are a number of ways in which tracers can be labelled. However, before the tracer can be labelled, a suitable radionuclide should be identified. The choice of radionuclide depends on the amount of radioactivity needed for the experiment. According to Fan et al. (2006a), tracking efficiency and the extent to which PEPT data represents the system is dependent on the amount of radioactivity in the tracer. In addition, the tracer should be suitable, in terms of physical and chemical properties (Fan et al., 2006a; Parker and Fan, 2008). Thus, the tracer should be similar to the rest of the population it is meant to represent. 


\subsubsection{Radioisotopes used for labelling tracers}

There are a number of radioisotopes that can be used to label a tracer particle. These have half-lives ranging from a few minutes to days, or even years. When considering the selection of a radioisotope to be used in a PEPT experiment, there are two issues to consider that are related to half-life (Fan et al., 2006a). Firstly, the half-life should be short enough to avoid radioactivity persisting long after the experiment. This is because it may be difficult to separate the tracer from the bulk, when need be. Secondly, the half-life should be long enough to warrant sufficient radioactivity during long experiments - which are usually desirable for ergodicity arguments.

The most commonly used radionuclide in PEPT work is ${ }^{18} \mathrm{~F} .{ }^{18} \mathrm{~F}$ has a half-life of about 110 minutes (Parker et al., 1997b), which makes it very convenient. In addition, ${ }^{18} \mathrm{~F}$ decays only by positron emission and, therefore, has no interfering $\gamma$-rays (Parker et al., 1993). Other possible radionuclide include: ${ }^{61} \mathrm{Cu}$ and ${ }^{66} \mathrm{Ga}$. In this work, the radioisotope used in tracer labelling is ${ }^{18} \mathrm{~F}$, due to its convenient half-life.

\subsubsection{Labelling techniques}

A number of techniques exist that are used for the labelling of tracers particles. An elegant summary can be found in Parker and Fan (2008). These can be summed up in three groups: direct activation, ion-exchange and surface modification. In direct activation, the tracer particle is bombarded with a high energy beam from a cyclotron. Irradiation with a $33 \mathrm{MeV}$ ${ }^{3} \mathrm{He}$ beam yields ${ }^{18} \mathrm{~F}$ for substances containing naturally occurring oxygen (like glass beads) or ${ }^{64} \mathrm{Cu}$ when the target material is stable copper fragments (Parker et al., 1997b). To ensure sufficient radioactivity is achieved by bombardment, the particle must be at least $1000 \mu \mathrm{m}$ (Fan et al., 2006a). This is because radioactivity achieved is proportional to the cross-section area of the particle. Accordingly, if the particle is less than $1000 \mu \mathrm{m}$, the radioactivity achieved is too low for accurate tracking.

In ion-exchange, the radionuclide is produced in solution form and then transferred into a resin bead (Parker et al., 1997b). In the case of ${ }^{18} \mathrm{~F}$, the radioisotope is produced as a solution of Fluoride ions (in deionised water). Thereafter, the ${ }^{18} \mathrm{~F}$ can be used to exchange with the counter-ions in resin beads, which act as ion-exchange media. This method can be used to produce much smaller tracers of about 200-600 $\mu$ m (Fan et al., 2006a,b). 
The third method used in the labelling of particle tracers is known as surface modification. This summary is based mainly on Fan et al. (2006b). Surface modification is used to improve the selective adsorption of chemicals on a given surface. What is modified in this case is the surface chemistry of the particle that is to be labelled. Normally, metallic ions may be introduced to the surface of the particle. Anions then bind with the metallic ions on the modified surface. $\mathrm{Fe}^{3+}, \mathrm{Pb}^{2+}$ and $\mathrm{Cu}^{2+}$ are commonly used metallic ions for this purpose. However, $\mathrm{Fe}^{3+}$ is favoured of the three, due to its chemical activity and its ability to adsorb onto most solid surfaces. The addition of the metallic ions onto the solid surface results in an increase in the adsorption of ${ }^{18} \mathrm{~F}$ (Fan et al., 2006b). For example, Figure 4-1 shows the effect of $\mathrm{Fe}^{3+}$ on ${ }^{18} \mathrm{~F}$ adsorption on quartz.

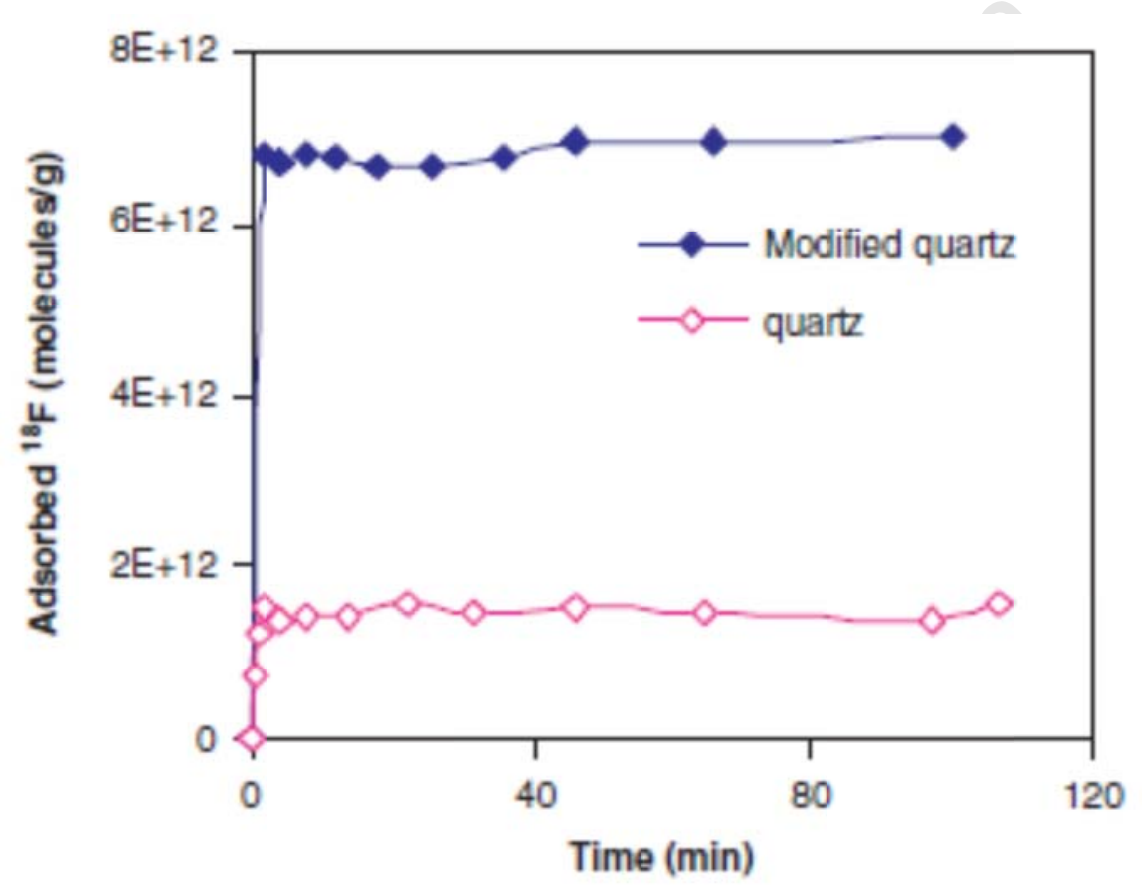

Figure 4-1 Effect of $\mathrm{Fe}^{3+}$ on ${ }^{18} \mathrm{~F}$ adsorption on quartz (Initial ${ }^{18} \mathrm{~F}$ concentration: $\left.6.8 \mathrm{X} 10^{9} \mathrm{M}\right)($ Fan et al., 2006b)

Based on Figure 4-1, the addition of $\mathrm{Fe}^{3+}$ ions onto a solid surface causes a dramatic increase in the adsorption of ${ }^{18} \mathrm{~F}$. For instance, under optimised conditions, increases from 2 to 400 $\mu \mathrm{Ci}$ in the radioactivity adsorbed in a single quartz particle have been observed (Fan et al., 2006b). Thus, surface modification can be used to considerably extend the range of particles that can be radio-labelled.

In this work, the particles studied needed not to be very small. Thus, the direct activation method was sufficient as a labelling technique. 


\subsubsection{Cameras: Detection}

The cameras used in detecting the location of the tracer vary in design. The PEPT facility at the University of Birmingham uses a pair of parallel detectors. The Birmingham camera has been evolving over the years, and has improvement quite significantly in terms of accuracy and precision. The new PEPT facility at iThemba Labs in Cape Town, South Africa, uses a series of detectors arranged in ring configuration around the systems being studied. In this work, the University of Birmingham facility was used. Thus, more attention is given to that camera.

\subsubsection{Initial Camera}

The Birmingham positron camera was constructed in 1984. This camera was designed for the application PET to engineering studies. Descriptions of the camera can be found in Hawkesworth, et al. (1991) and Parker et al. (1993, 1994). The camera consists of a pair of multi-wired proportional chambers, operated in coincidence. The chambers have a sensitive area of about $600 \times 300 \mathrm{~mm}^{2}$. They also consist of cathode planes separated by anode wires at a potential of $3.5 \mathrm{kV}$. Each plane made of lead-approximately $50 \mu \mathrm{m}$ thick-is divided into parallel strips. These planes also serve as the principal converter of protons.

When the high energy photons strike the planes, an electron is released into the gas (usually a mixture of isobutene or Freon) which fills each chamber. This initiates a Townsend avalanche adjacent to a nearby anode wire. This is detected as a voltage pulse on the anode and on the two neighbouring cathodes. Due to the orthogonal directions of the cathodes on either side of the anode, the position of the $\gamma$-ray interaction can be located in both $x$ and $y$ coordinates. One of the major limitations surrounding the PEPT camera described above is the size of equipment that can be put between the two detectors. Thus, many improvements have been done to the original camera.

\subsubsection{Moving cameras}

This section is based mainly on work done by Parker et al. (1997b). To extend its axial view, the positron camera can be mounted on rails. The camera can then be moved by a motor, with an absolute shaft encoder, used to read out the position of the translation stage. Whenever the tracer position is approximated to be too far from the axial centre of the detector, the computerised motor moves the camera towards the tracer, such that the tracer is again 
completely within the axial field of view of the camera. The shaft encoder values are used to correct the translation stage's coordinates into the laboratory frame of reference.

Thus, automatic tracking of the tracer can be done quite accurately, provided the axial motion is not very violent. This can increase the axial view from only $25 \mathrm{~cm}$ to $145 \mathrm{~cm}$ (Parker, et al., 1997b). With this arrangement, however, come errors resulting from the movement of the translation stage. However, further analysis has demonstrated that the uncertainty caused by the motion of the translation stage is negligible for most studies that would need such a camera (Parker et al., 1997b).

\subsubsection{An improved camera}

The major limitation of the original PEPT camera, and its earlier variations, was its low quantum efficiency in detecting $511 \mathrm{keV}$ annihilation photons, resulting in a limited coincidence event rate (McNeil et al., 1994). An improved camera is shown in Figure 4-2 (Parker et al., 2002).

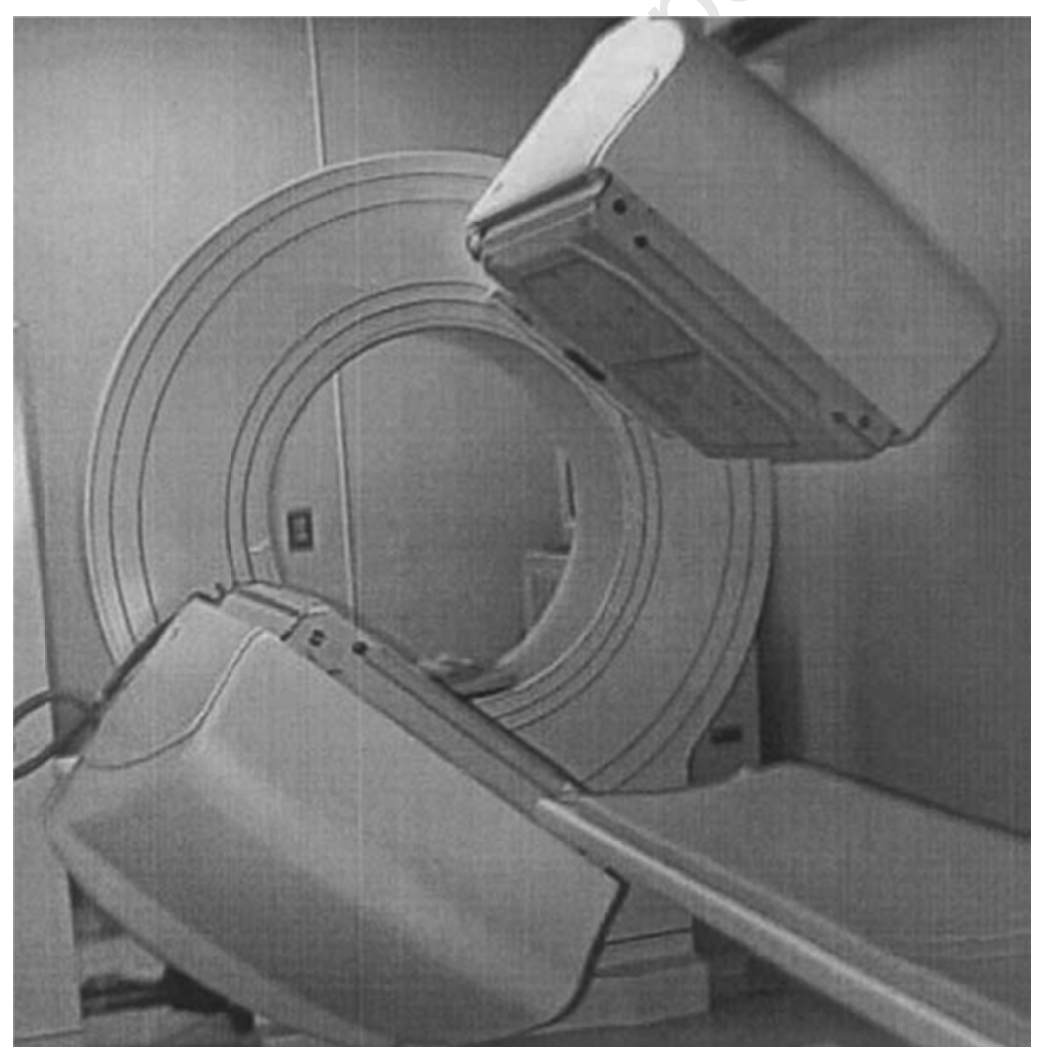

Figure 4-2 The new Birmingham positron camera (Parker et al., 2002)

The camera consists of a pair of detector heads on a motorised gantry. The heads can be rotated about the horizontal axis and have a variable gap (from 250 to $800 \mathrm{~mm}$ ) between 
them. The heads are operated in coincidence where the resolving time is about $7.5 \mathrm{~ns}$. The resolving time is the maximum time between two detectors on either heads that is required to establish a coincidence event. Each head comprises a single $16 \mathrm{~mm}$ thick Sodium Iodide crystal, approximately $590 \times 470 \mathrm{~mm}^{2}$. The crystal is optically coupled to an array of 55 tubular photomultipliers. Each photomultiplier is connected to a separate ADC. The 55 ADC channels are controlled by a single board computer in the head.

Whenever a scintillation occurs within the crystal, its centroid is determined using built-in software. Besides the flexibility of using software, compared with using analogue circuits, higher data rates probably count for the major benefit of using this camera. In particular, data rates can be about a factor of 20-40 higher, compared with the old camera (Parker et al., 2002). The new camera also has quite an impressive energy resolution. Location precision is also about 10 times better for the new camera (Parker et al., 2002).

Thus, there is greater value in the data obtained using this camera than the older ones. Consequently, studies of particle packing, which in previous studies have had mostly qualitative value, can be used for quantitative analysis. Thus, PEPT is a viable option in the study of porosity in rotary mills.

\subsubsection{The detection process}

During $\beta^{+}$decay, a positron is emitted which annihilates upon contact with an electron. This process produces a pair of collinear $511 \mathrm{keV} \gamma$-rays. These rays are captured by the positron camera discussed above. However, the useful events in the tracking of the tracer are those involving the simultaneous detection of a pair of $\gamma$-rays. Thus, only those $\gamma$-ray pairs that are detected within a given delay time (resolving time; approximately $7.2 \mathrm{~ns}$ ) are considered to represent a coincidence event. The information captured by each coincidence event can be thought of as a line represented by the two detection points in the camera and is formally referred to as a line of response (LOR). Each LOR is expected to pass very close to the position of the tracer at a given time, $t$. During an experiment, this data is logged onto computer files for further processing.

However, this includes all coincident events that occurred during run time. During a PEPT experiment, there is usually a large number of coincidence events recorded. Of these events, a significant number (the majority) are actually corrupt (Hawkesworth et al., 1991). The corrupt events may result from either random or scattered pairing of the emitted $\gamma$ rays. Thus, 
most of the events do not depict the true position of the tracer at any given time. Consequently, the raw data is not very useful for immediate analysis. Pre-processing is, therefore, a mandatory requirement before the data can be used in any quantitative modelling context.

To establish the true location of the tracer entails eliminating the corrupt "lines", so that only "true" detections can be used. This is done via a location algorithm, which has been discussed quite extensively (Hawkesworth et al., 1991; Parker et al., 1993, 1994; Parker and Fan, 2008).

\subsubsection{Software: The location algorithm}

To ascertain the tracer's location, a process known as triangulation is applied, iteratively. In triangulation, the location of a point is determined by measuring angles to that point from known points. Equivalently, perpendicular distance $\delta$ from a centroid point (initially unknown) to the selected LOR's can be used. The centroid point is the approximate location of the tracer. For multiple coincidence events, the perpendicular distance from each LOR is a measure of the error with which that line approximates the position of the tracer. The sum of these errors gives the compound error associated with the number of events being used in that approximation. The location algorithm then seeks to minimise this error by discarding corrupt events in an iterative scheme.

Firstly, a number of sequential events $N_{e}$ are chosen. $N_{e}$ depends on the speed of the tracer. However, it must be is large enough to allow for the elimination of corrupt detections and still give a sufficient data set for estimating the particle position. $N_{e}$ must also be small enough to allow for the tracer not to have moved significantly over time $\Delta \mathrm{t}=\mathrm{t}_{\mathrm{Ne}}-\mathrm{t}_{1}$.

Based on the chosen $N_{e}$ events, an initial approximation of the tracer location is computed. If this process is applied iteratively, a number of corrupt events can be discarded in each step based on how much they contribute to the compound error. The lines furthest from the approximation are expected to be the worst estimates. Therefore, if the perpendicular distance for a given line is greater than the value $k \delta$, for a chosen $k$, that line is discarded. Thereafter, the location is recalculated based on the remaining lines, resulting in a better approximation.

The process continues until the number of events remaining is equal to a given fraction $f$ of the initial number $N_{e}$ of events. Ultimately, a significant number of corrupt events are 
discarded. Thus, the remaining (presumably) uncorrupted lines converge at a point very close to the location of the tracer (within camera resolution), as shown schematically in Figure 4-3.

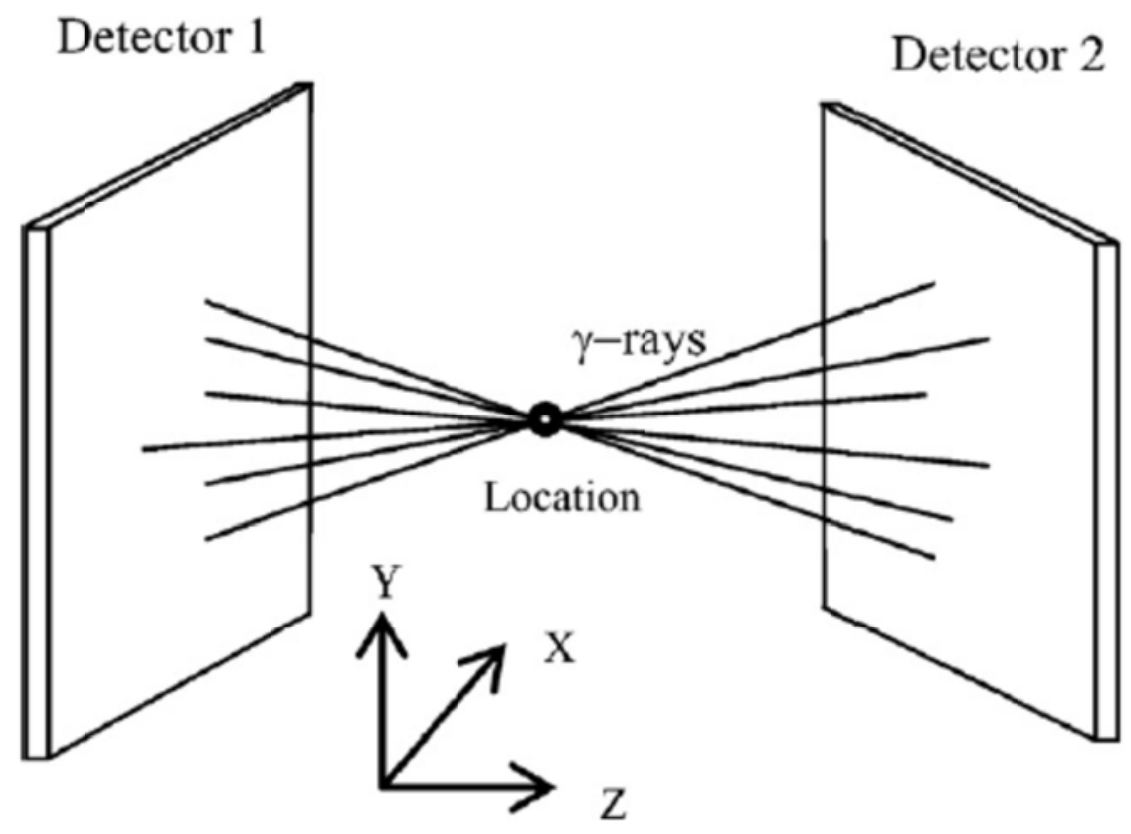

Figure 4-3 The convergence of lines at a centroid region representing particle position

Once a location has been determined, the time for that location is also determined. This is done by finding the average time in the final set of events remaining after establishing the tracer location. The final error in the approximation and the remaining number of events are also documented. The algorithm then continues on to determine the next location by the same process above. This continues until the whole data set has been traversed. The result of the whole process described above is a data file consisting of position, time and error, with respect to time. Figure 4-4 shows a snapshot of a typical data file. 


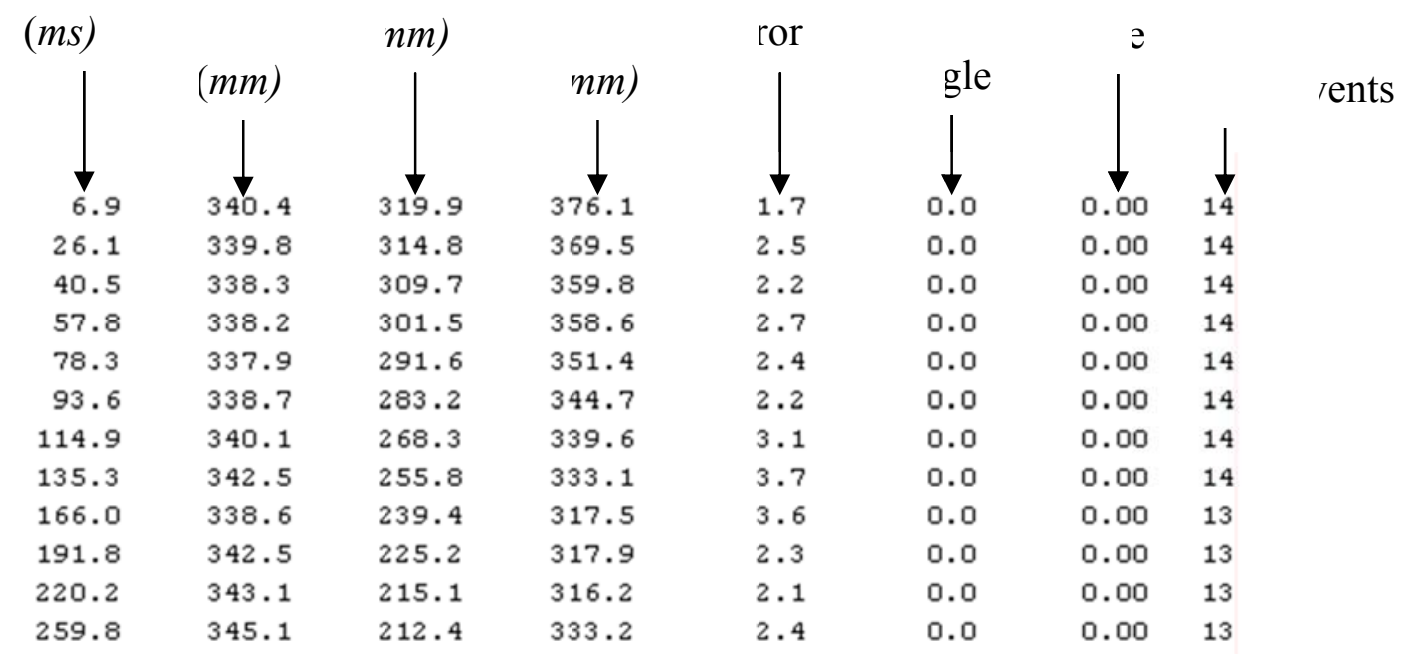

Figure 4-4 Sample PEPT trajectory data

The coordinate system used in the file represented above is based on the positron camera coordinate system. It is this file that is then used in analysing the dynamics of the particulate system under study.

\subsubsection{How valuable is PEPT data?}

From the discussions above, very detailed (particle scale) measurements of the dynamics of a system can be performed using PEPT. The data gathered in the process can be used to determine quantities such as velocity, acceleration, particle packing characteristics and other quantities that depend on particle position as a function of time. These quantities are difficult to measure using conventional methods.

Thus, PEPT opens new avenues of particle level research and provides information that is useful in optimising equipment design and operations. However, the information that is collected using PEPT is limited to a single particle's trajectory. Accordingly, interpreting such data into information about the whole system is nontrivial. Fortunately, a number of challenges related to this problem have already been addressed adequately. Some of these are discussed shortly. However, there are still a significant number of challenges that are yet to be solved. The next section discusses the use of PEPT in the study of packing density and porosity in dynamic systems. 


\subsection{Packing characterisation using single particle data}

\subsubsection{Defining measures through single particle tracking}

The use of single particle tracking to quantify physical properties of particulate systems requires the use of statistical mechanics. Statistical mechanics is a subject dedicated to understanding the physical behaviour, where only limited information is known about manyparticle systems (Bloch and Walecka, 2000). This requires a link between single particle trajectory data and the behaviour of the whole particle assembly. This link, between microscopic dynamics and macroscopic thermodynamics, via an invariant probability distribution forms the prescription for equilibrium statistical mechanics (de Oliveira and Werlang, 2007). Justifying such a prescription is a nontrivial task.

However, the ergodic hypothesis intervenes in such problems. This hypothesis allows for the exchange of time averages with space averages. A detailed treatment of this subject is presented in the next chapter. For now, consider what would be required to study the packing of particles in a dynamic system in equilibrium.

Ideally, measuring porosity from a fundamental view point requires knowledge of the positions and sizes of all the particles in a given space. This would demand that the system being studied is either stationary or at equilibrium and that porosity is invariant with time. In the equilibrium case, the calculation would require different realisations (or snapshots) of the system taken at different angles, in order to get the full picture. These realisations compose what is known as an ensemble - a set of possible states for the system. An average taken on an ensemble is known as ensemble average. Conversely, fixing the realisation index and taking the measurements over a period, time averages can be used to estimate system properties. Unfortunately, knowing the positions of all particles is not possible in reality. Thus, determining information about the whole system requires the use of other forms of statistics.

In statistics, a sample is usually used to represent a bigger population. Based on analysis of the sample data, conclusions can be made about the whole population, provided the sample represents the whole system suitably. One way to increase the chances of achieving representativeness is by taking a bigger random sample. The sample in the case of a particulate system could be a chosen number of particles. However, when it is not possible to study many particles (as is the case with PEPT) a smaller number of particles could be used. 
In a dynamic system, a smaller number of particles can be studied, since particles can represent similar particles by moving around the system for a long period. It follows naturally that, for a smaller sample, a longer observation time would be required. If the observation time is infinitely long, a single particle would be enough. Thus, to ensure that a single particle can be used adequately, the particle should be studied for a sufficiently long time.

If the system is ergodic, the tracer particle is expected to traverse essentially all possible states in which all other particles can be found, if tracking time is sufficiently long. Geometrically, the tracer would be expected to visit almost all possible positions other particles would visit during the observation period. Thus, time averaged definitions of single particle quantities can be used to represent system level properties. Hence, a single particle can represent a larger system, which it is part of.

\subsubsection{Calculating the packing fraction of a monosized system}

Based on the discussions above, it is possible to calculate particle packing properties using PEPT. Packing density has been characterised for a fluidized bed using single particle tracking data (Wildman et al., 2000). A part of the work by Wildman et al. (2000) was aimed at characterising packing density profiles in a vibrofluidized bed of spheres. Thus, the container volume was initially divided into volume elements.

Based on the ergodicity of the system, it is assumed that the residence time distribution of a tracer is proportional to packing density distribution of the whole system (Parker et al., 1997a; Wildman et al., 2000). In this context, residence time is the amount of time a particle spends in a given region or volume element of a system. The fraction of total time a tracer spends in a given volume element is called fractional residence time. The fractional residence time is equal to the probability of locating particles in a volume element and is proportional to packing density. The proportionality of residence time and packing density is also consistent with theory, as described in Arnold and Avez (1989, p134). The location probability can also be based on the fraction of locations that a particle is found in a given volume element.

However, a more accurate representation of packing density is to weight the location probability density by the time spent in each volume element. The need for weighting arises from the fact that PEPT data has variations in inter-location time intervals. Residence time is calculated by appropriating fractions of the time between two consecutive locations to each 
of the volume elements the tracer traverses. If the tracer remains in the same volume element, the time is added to that volume element. Ultimately, the total time in each volume element is then calculated and normalized to a total on all volume elements.

The number density is given by:

$$
n(x)=\frac{N F(x)}{V_{s}}
$$

Equation 4.1

where $N$ is the number of particles in the entire system, $F(x)$ is the residence time fraction and $V_{s}$ is the volume of a volume element. The packing density $\eta(x)$ can then be calculated by multiplying the number density by the volume of one particle:

$$
\eta(x)=n(x) \frac{\pi d^{3}}{6}
$$

Equation 4.2

where $d$ is the particle diameter. Thus, porosity can be calculated easily by $\varepsilon(x)=1-\eta(x)$.

Wildman et al. (2000) provided justification for this approach by way of a mathematical proof, making use of the ergodicity of the system. The proof is such that: if a segmented system of particles were assumed to be stationary, and its stationary state assumed to be ergodic, the mean number density (given by the fraction of particles $N_{j}$ in volume element $j$ ) would be expressed as a time average of a dynamical variable along the phase space trajectory of a single sample (particle). This result justifies the calculation of packing fraction of a monosize system. Phase space is simply the representation of a system by a set of position and momentum coordinates of all $N$ particles in the system. The details of the mathematics of the ergodic hypothesis are presented in the next chapter.

The method presented above was developed for a monosized system. On the other hand, rotary mills usually contain charge with a particle size distribution. Therefore, there is need to extend the method to quantify porosity of multi-component systems.

\subsubsection{Multi-component systems}

A general assumption in the use of PEPT is that, a tracer can only represent particles similar to it. Thus, a number of particles of various sizes would need to be tracked in the case of 
multi-component systems. The packing density of a binary fluidized system has been studied previously (Wildman and Parker, 2002). In this case, coexistence is assumed in order to extend the packing density estimation method to a two-component system.

Coexistence implies that the different size components can each be studied separately despite being in the same system. Thus, tracers of different sizes-each representing a size component - are tracked, in turn. The trajectories of the two tracers are each used to study the behaviour of their respective component in a binary mixture. That way, it is possible to compute the packing density and granular temperature for each component (Wildman and Parker, 2002). As expected, the different particles give different packing density profiles. This result is useful in motivating the use of different sized tracers each representing a size component.

In the work by Wildman and Parker (2002), the packing densities of the two size components were treated separately. To compute the packing density (or porosity) of the binary system, the results of tracking the different tracers must be combined. How this combination is done was a key question in this thesis. To the best of my knowledge, there is no method for computing the porosity of multi-component mixtures, using PEPT data. If this is addressed, PEPT can be used effectively to measure the porosity of the tumbling charge in rotary mills. Part of the aim of this thesis was to extend the methods discussed above to include multicomponents. Before venturing on that path, consider the limitations of the PEPT method discussed in next section.

\subsubsection{Limitations of the PEPT technique}

\subsubsection{Equipment size}

One of the limitations of the PEPT facility is the size of equipment that can be placed between the detectors. Currently, the two detector chambers have an active area of $590 \times 470$ $\mathrm{mm}^{2}$ each and are separated by a variable gap of between 250 and $800 \mathrm{~mm}$ (Parker et al., 2002). Therefore, only a laboratory scale mill could be used in this work. This was not a major drawback, however. Much of research done on fundamental aspects of industrial processes has been at either laboratory scale or pilot scale. This has always been in view of the possibility of scale up on the results. Scale up is not addressed directly in this work. Otherwise, regarding the objectives of the thesis, the main aim has been to develop the methods for studying multi-component mixtures within a rotary mill using non-invasive 
particle tracking. Thus, size limitations were not a major issue in this work. Once the techniques are in place, it should be possible to think about how they can be applied to bigger systems. This is in view of increased field of view of the positron camera, allowing the study of bigger equipment.

\subsubsection{Error and uncertainty}

From the discussions on the PEPT technique and the location algorithms used, error is inevitable. As indicated in Figure 4-4, one of the columns is dedicated to error. The error recorded in that column is a result of the location algorithm. This is due to the algorithm stopping its location determination process once the preset fraction of initial events used in the estimation is reached. Thus, the error associated with locating the tracer should be expected. For a given spatial resolution of $W$, and a fraction $f$ of initial events $N$ used in estimating a given location, the accuracy of location is proportional to $W / \sqrt{f N}$ (Parker et al., 2002).

\subsubsection{Multiple particle tracking}

The current PEPT facility can only track up to three particles, accurately (Parker and Fan, 2008). However, Parker and Fan have observed that the three-particle tracking is less precise than standard PEPT. This is attributed to the challenges in resolving the locations of tracers using essentially the Birmingham single particle location algorithm. Furthermore, threeparticle tracking cannot be used at comparatively high speeds.

In this work, only single-particle tracking is used, since this is considered sufficient. This is due to the capability of the PEPT facility to track a particle for a long period, resulting in accumulation of essentially whole-system data. Furthermore, whether one tracks one or three particles at the same time makes no difference for studies of particle packing. Theoretically, we can reproduce the data from tracking three particles by tracking one particle three times and for three times longer. The difference would only become significant if a very large portion of particles in the system can be tracked at the same time. However, this is a very difficult problem, at the current state of development.

\subsubsection{Possibilities of improvement}

One important challenge is how to deal with multi-component mixtures. Important questions that were asked include: is it possible to improve upon the techniques presented in the preceding sections to include multi-components? It should be possible to extend the methods. 
Firstly, it is clear that one can study a multi-component system by tracking differently sized tracers. What is required is a way to combine the results of tracking the different tracers. Other possible improvements are related to the PEPT technique itself. With the technique becoming more popular, it will become necessary to study bigger systems and smaller particles. Both of these are areas of extensive research presently.

\subsection{Conclusion}

The method used to study the particle dynamics in a laboratory mill, in this work, is PEPT. The PEPT technique has wide application in the study of dynamic systems. In particular, it can be used effectively in characterising particle packing, despite only tracking one particle. This has been done successfully on monosized and binary systems (for individual components). This is based on certain assumptions, such as ergodicity and coexistence. However, to the best of my knowledge, there is no method for calculating porosity in multicomponent mixtures, using PEPT. The major challenge, therefore, is to extend the methods that exist for monosized systems to include multi-components. 


\section{Mathematical formulations}

This chapter presents the process of formulating mathematical techniques used to compute the porosity of multi-component mixtures in rotary mills. It also discusses fundamental assumptions made in the formulating process. The formulations presented here are an extension of well established techniques for computing packing fraction in monosized systems, which were discussed in the previous chapter.

\subsection{Assumptions}

To formulate techniques for computing porosity of multi-component mixtures, three assumptions were made. The first assumption is that the system under study is at steady state throughout the duration of an experiment. Steady state is a necessary condition, without which the idea of time averaging would not be meaningful. The second assumption is that, the system being studied is ergodic (essentially). Thus, single particle (or tracer) data can be used to study the 'system'. In this case, particularly, system refers to what the tracer represents. In a multi-component system, this could be all particles similar to a tracer particle - in terms of size, for example. Thus, in order to study the whole system, another assumption is made. The third assumption is that size components in the system coexist in a way that each size component exhibits ergodic properties. In other words, the system is assumed to satisfy the coexistence property.

These three assumptions are not unique to this work. They have also been made by other researchers involved in PEPT work, which were discussed, briefly, in the previous chapter. For example, Wildman et al. (2000) assumed that the fluidised bed they studied was ergodic and at steady state - equilibrium. In addition, Wildman and Parker (2002) assumed the binary mixture they studied exhibited coexistence properties. What follows is a discussion on the fore-mentioned fundamental assumptions. This is in view of their application to porosity computation in multi-component mixtures within rotary mills, using single particle PEPT trajectory data.

\subsection{Ergodicity}

Ergodicity is a property of dynamical systems that provides for the exchange of ensemble averages with time averages. Thus, a time averaged quantity along the tracer's trajectory can be studied and used in quantifying properties of a system within which the tracer is studied. For example, from the previous chapter, the fractional residence time of a single particle is 
used to calculate packing density (Wildman et al., 2000; Wildman and Parker, 2002). Justification for this approach exists and can be found in Wildman et al. (2000). This section discusses the concept of ergodicity. Some aspects of ergodic theory are also discussed, including necessary conditions for ergodicity. Issues around application of the theory to the particle packing problem are also presented. Furthermore, aspects of mapping PEPT data to abstract ergodic theoretic concepts and notation are also discussed.

\subsubsection{The ergodic theory}

The ergodic hypothesis was first proposed by Ludwig Boltzmann in an effort to explain the basis of statistical mechanics. To study the ergodic properties of a given system requires understanding of the ergodic theory. This branch of mathematics came about as a result of efforts to interrogate the foundations of statistical mechanics. Ergodic theory is the study of the qualitative properties of actions of groups on spaces (Walters 1982, p 1), or the study of the statistical properties of dynamical systems (Brin and Stuck 2002, p 69). The space in the definition by Walters has some structure-it can be a measure space, for example. Each element of the group acts as a transformation on the space and preserves that structure. In particular, each element acts as a measure-preserving transformation. Ergodic theory is concerned with such measure-preserving transformations. Some preliminary concepts in measure theory are discussed next.

\subsubsection{The concept of measure}

This summary is based on Walters (1982) and Brin and Stuck (2002). Consider a set $X$, and a $\sigma$-algebra $\beta$ of subsets of $X$. $\beta$ is a non-empty collection of subsets of set $X$ closed under complements and countable unions (and intersections), where (1) $X \in \beta, \varnothing \in \beta$;

$D \in \beta \Rightarrow X \backslash D \in \beta$; and (3) If $D_{n} \in \beta$ for $n \geq 1$ then $\bigcup_{n=1}^{\infty} D_{n} \in \beta$. A measure on $\beta$ is defined as a function $\mu: \beta \rightarrow \square^{+}$(non-negative). Thus, $\mu$ assigns a real number to each set $D \in \beta . \mu$ must satisfy the following conditions: (1) $\mu(\phi)=0$; (2) Monotonicity: $\mu(C) \leq \mu(D)$ for all $C, D \in \beta, C \subset D$; and (3) $\sigma$-additivity: $\mu\left(\bigcup_{i} A_{i}\right)=\sum_{i} \mu\left(A_{i}\right)$ for any countable collection of disjoint sets $A_{i} \in \beta$.

From the foregoing definition, measure has to do with size, volume and other related quantities. For example, in a real line interval $X=[a, b]$, measure could be interval length 
$\mu(X)=b-a . \beta$, in this case, would consist of subintervals on $[a, b]$. Thus, if $\mu$ was defined generally as interval length, then $\mu$ of a given subset of $\beta$ gives the length of the subinterval related with that subset.

A set of zero measure is called a null set. Conversely, a set has full measure if its complement is a null set. The pair $(X, \beta)$ is called measurable space and the triple $(X, \beta, \mu)$ is known as measure space. If $\mu(X)=1,(X, \beta, \mu)$ is called a probability space and $\mu$ is called a probability measure. However, whenever $\mu(X)$ is finite, a probability measure can be obtained by rescaling (normalizing) $\mu$ by the factor $1 / \mu(X)$. Thus, if $X$ was a set of particle positions for a given system, $\mu$ can be the probability that a particle belongs to a given set $A \in \beta$, based on certain criteria. In this study, attention is given to what are called measurable sets and to probability measure. $C \in \beta$ is measurable if and only if (1) its compliment $X \backslash C$ is measurable; (2) $\mu(C)+\mu(X \backslash C)=\mu(X)$.

\subsubsection{A dynamical systems setting and observables based on measure}

The proper setting for ergodic theory is a dynamical system $\left(X, \beta, \mu, T_{t}\right)$. Given a system of $N$ particles (each having $n$ degrees of freedom) with position vector $q=\left(q_{1}, \ldots, q_{n N}\right)$ and momenta vector $p=\left(p_{1}, \ldots, p_{n N}\right)$, the dimension of the phase space $\Gamma$ is $2 n N$. If $x$ is a point in the state space at time $t_{0}$ and $T_{t}$ is a transformation $T_{t}: \Gamma \rightarrow \Gamma, T_{t}(x)$ denotes the point representing the system at time $t_{0}+t$.

A dynamical system induces an action on functions: $T_{t}$ acts on a function $f$ by $\left(T_{t} f\right)(x)=f\left(T_{t}(x)\right)$ (Brin and Stuck, 2002, p. 73). Of interest is the asymptotic behaviour of the system. $T_{t}$ is measure-preserving if $\mu\left(T^{-1} A\right)=\mu(A)$ for every $A \in \beta$. In this case, the set $A$ and measure $\mu$ are invariant under $T_{t}$. Ergodic theory is concerned with systems of invariant sets - and invariant measure.

In physical terms, usually, a macroscopic physical quantity — an observable — can be defined. Some observables, such as volume, can be defined directly from the macroscopic variables of a system, such as length, width, radius and height. Conversely, other quantities, such as porosity and temperature, are presumably defined by a real-valued function- $f: \Gamma \rightarrow \square . f$ is defined on $X$-a set of microscopic variables — via an invariant probability measure $\mu$ (de 
Oliveira and Werlang, 2007). Thus, an observable can be defined based on fundamental variables, such as displacement and momentum, at a microscopic scale.

\subsubsection{The ergodic hypothesis}

Consider a measure space function $f$, corresponding to an observable. The time average is given by the following:

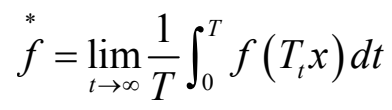

Equation 5.1

where $T$ is the total time of observation.

The ensemble average is given by:

$$
\bar{f}=\int_{X} f(x) d x
$$

Equation 5.2

The assumption in the time average calculation is that the limit exists almost everywhere and is independent of the initial position $x_{0}$. The ergodic hypothesis asserts the equivalence of the two averages above. Thus, if a system is ergodic, then:

$$
f^{*}(x)=\bar{f}(x)
$$

Equation 5.3

This implies that the time average of a single realisation of the system can be used to estimate the ensemble average (de Oliveira and Werlang, 2007). Therefore, a single particle can be studied over a period and its time-average properties used to estimate an ensemble average of a property of various realisations. These realisations, in the context of particle dynamics, can be particle trajectories. If a single particle is studied over a considerable length of time, the particle essentially visits all open sets of the phase space in the measurement process (de Oliveira and Werlang, 2007). Therefore, it is possible to get the coordinates of a single particle's orbit tracked for a considerable period and applied to the whole system. Furthermore, invariance in measure can still be achieved, at least asymptotically. It is this invariance that allows us to employ the ergodic hypothesis. 


\subsubsection{Residence or sojourn time}

The residence time $\tau(T)$ is the amount of time which an orbit spends in a given segment or measurable subset $A$ (Arnold and Avez, 1989, p. 134):

$$
\tau(T)=\text { measure }\left\{t: 0 \leq t \leq T, T_{t} x \in A\right\}
$$

\section{Equation 5.4}

In physical terms, the subset $A$ would represent a volume element (or region). In that case, residence time would imply the amount of time a particle spends in the given region of a system. According to Arnold and Avez, an abstract dynamical system $\left(X, \beta, \mu, T_{t}\right)$ is ergodic if, and only if, $\tau(T)$ in an arbitrary measurable set $A$ of an orbit $\left\{T_{t} x: 0 \leq t \leq T\right\}$ is asymptotically proportional to the measure $\mu$ of $A$ :

$$
\lim _{T \rightarrow \infty} \frac{\tau(T)}{T}=\mu(A)
$$

\section{Equation 5.5}

for all measurable $A$ and almost every initial point $x \in X$.

This theorem also justifies the use of residence time fraction to estimate packing density in a given volume element. Moreover, particle density depends entirely on particle position (de Oliveira and Werlang, 2007), which is given by a particle trajectory in PEPT data. The residence time fraction is equivalent to the probability measure $\mu$, equal to the probability of locating a particle of a given size in a particular region of the mill.

\subsubsection{Establishing ergodicity}

\subsubsection{Metric transitivity and ergodicity}

A dynamical system is metrically transitive, metrically indecomposable, or irreducible when, for any two sets $A, B \in X$, if $\mu(A), \mu(B)>0$, there exists an $n$ such that $\mu\left(T^{-n} A \cap B\right)>0$ (Shalizi 2007, p 205). It can be shown that metric transitivity implies ergodicity. A system is ergodic if and only if the only measurable sets invariant under $T_{t}$ have probability measure 0 or 1 (zero or full measure). In other words, set $A$ is invariant if and only if that set either 
represents a whole set's behaviour or has zero measure. Thus, if set $A$ has invariant measure, then either $\mu(A)=\mu(\varnothing)$ or $\mu(A)=\mu(X)$.

This implies that any one trajectory is everywhere dense and that measure does not depend on the initial value of the variable. Any such trajectory would, therefore, represent the entire system. In addition, if only such a trajectory was known, it can be studied in place of the entire system. Geometrically, the tracer visits all possible states other particles could ever be in within a given system, essentially.

\subsubsection{Decomposable systems}

If, however, a set $B$ has invariant measure $\mu(B)$ not equal to $\mu(\varnothing)$ or $\mu(X)$, the system is non-ergodic. Thus, the only set with non-zero measure that is invariant is the whole set (de Oliveira and Werlang 2007). Set $B$ above implies that, even though the trajectory has invariant measure, choosing it to represent the system would give wrong results - not equal to full measure. Geometrically, a trajectory visits a subset of the system in a biased manner. If this where a tracer's trajectory, the tracer might occasionally be stuck in one region or another for considerable periods. Thus, the trajectory may exhibit invariance, but would not appropriately represent the whole system. Such a system is called decomposable. Decomposable systems are not ergodic.

\subsubsection{Proof of ergodicity}

To prove that a system is ergodic requires establishing the existence of a unique invariant measure. The uniqueness requirement implies that, for an ergodic system, the only invariant functions are constants. Decomposable systems fail this test.

To prove that a system set $X$ is non-ergodic, one would test all sets in the $\sigma$-algebra $\beta$. If there exists one set that is invariant under transformation $T_{t}$, but with measure not equal to full measure, then the system is decomposable - and, therefore, non-ergodic. If, however, no set exists that is invariant under $T_{t}$ which has measure not equal to zero or full measure, then the system is ergodic. This implies that whichever trajectory is chosen to represent the system gives a unique invariant measure - a constant. Taking limits on the ergodic system, invariance means that a function $f(x)$ related to measure $\mu$ converges to a limit $L$. Uniqueness implies that $L$ is unique. To illustrate this, consider two sets $A, B \in \beta$ representing two different trajectories. If $f$ is invariant on either set, such that: 


$$
\lim _{t \rightarrow \infty} \frac{1}{T} \int_{A} f\left(T_{t} x\right) d x=L_{A}
$$

Equation 5.6

and

$$
\lim _{t \rightarrow \infty} \frac{1}{T} \int_{B} f\left(T_{t} x\right) d x=L_{B}
$$

Equation 5.7

then $f$ gives a unique result: $L_{A}=L_{B}=L$.

\section{Convergence}

Another way of stating the existence of a unique invariant measure is that, the function $f$, as $t$ approaches infinity, converges to a constant. Von Neumann (1932) showed that, for a physical system, it is possible to find a constant quantity $C$ (constant with respect to $T_{t} x$ based on $f$ ) which can be replaced by a time average. The key question that arises is: Can a constant $C$ be determined that the statistical dispersion about the time average is very small, i.e.

$$
\int_{X}|f-C|^{2} d v<\varepsilon
$$

Equation 5.8

where $\varepsilon>0$ is very small?

\subsubsection{Practical proofs}

Proving the ergodicity of a given system can be very difficult, especially if one has to test many possible invariant sets, in order to show that their respective measures converge to a constant. As a result of such difficulty, quasi ergodicity can be explored. Quasi ergodicity does not have as strong a claim, as strict ergodicity, on the equality of time averages to ensemble averages. However, the conditions presented above have been used in some studies to demonstrate ergodicity in physical systems. For instance, Duan and Goldys (2001) demonstrated the existence of a unique invariant measure in their study of geophysical flows. In addition, Sposito (1997) evaluated the ergodic assumption in a strictly dynamical sense. The study presented a stochastic approach to modelling the scale effect exhibited by solute plume spreading in an aquifer. 
In a paper by Wildman et al. (2000), a sufficient proof, in the physical sense, was presented. In this proof, the segments in the system were subdivided into smaller ones. The packing density was then evaluated for these smaller regions. Thereafter, the standard deviation was computed. Finally, ergodicity was shown by the very small standard deviation, in the smaller regions' values. This proof is useful to the current study, because it justifies the methods that will be presented later on.

Particularly useful to this current study work is the proof by Baumann et al., (1994). Baumann et al. demonstrated ergodicity in rotating drums. The authors compared the results of tracking a single particle to those of studying all the particles by computer simulation. Thus, if the ensemble average of a given process can be measured, then the deviation of the time average prediction can be assessed. If the two averages are not statistically different, conclusions can be made that time average equals ensemble average, essentially. Baumann et al. were able to establish this statistical equality in their work. Thus, based on the work by Baumann et al., we can be confident that the assumption of ergodicity can be applied to the study of rotary mills using the PEPT technique.

\subsubsection{Steady state}

Steady state is a way of defining a system in equilibrium. It implies that, a given quantity is invariant (essentially) within a given time frame. If a system changes significantly over time, the quantities measured in the process may not always be meaningful when one uses time averages. Thus, the steady state property is very important especially if a system is to be studied for a considerable length of time. In the case of a stochastic process, the system would need to have the recurrence property of dynamical systems and on average be at equilibrium. The time average would need to be equal to the equilibrium average (Lenci 2003). This is a variation of the ergodic hypothesis.

It is easy to show that a rotary mill is at steady state by studying the evolution of the power draw with time. The power is seen to converge after a short time to signify that the system is at steady state. Power draw is a good measure of steady state when particle packing is being studied. This is due to the dependence of both power draw and particle packing on particle position. Power draw is given by (Govender and Powell 2006)

$$
P=[M g \cos \theta] R \Omega
$$

Equation 5.9 
where
$M$ is the total mass of the charge,
$g$ is the acceleration due to gravity,
$(R, \theta)$ are the polar coordinates to the CoM (centre of mass) and
$\Omega$ is the average angular velocity of the mill.

In this work, the power draw was used in determining whether the system was at steady state. The details of this analysis are presented in the results section.

\subsection{Packing of mixtures}

The packing of mixtures has been studied widely (Ouchiyama and Tanaka, 1986; Yu and Standish 1987, 1991; Dias et al., 2004; Arntz et al., 2008). From such studies, key concepts can be understood that help in studying multi-component mixtures. For rotating drums, in particular, studies have been conducted which investigated porosity and other packing characteristics (Arntz et al., 2008). Some studies have focused on characteristics of multicomponent mixtures such as, mixing and segregation (Hill and Kakalios, 1994; Dury and Ristow, 1999; Ding et al., 2002). This section discusses important concepts in the packing of mixtures that can help in the characterisation of porosity using single particle packing techniques. Firstly, the property of coexistence is discussed. Thereafter, segregation and how this phenomenon relates with coexistence are discussed. The section ends with the rationale for tracking a particle from each size component in order to quantify properties relating to the whole system.

\subsubsection{Coexistence}

The property of coexistence implies that two or more size components can exist within a given volume. The coexistence property allows the subsystems to exhibit behaviour that can be studied independent of the other components, despite being in the same system. In other words, each component can be affected differently by measure-preserving transformations. It is possible to have coexistence of phases and components in a probability space (de Oliveira and Werlang, 2007). Thus, if coexistence holds for a given system, individual components can be ergodic and the whole system collectively ergodic. 
Consequently, we can estimate the statistical properties of a multi-component system by studying each component within the system. Coexistence also justifies the use of different sized tracers, in turn, as being representative of other particles within respective size components. It is, therefore, an important property in the study of multi-component systems using PEPT. The coexistence assumption has also been effectively applied to the study of a binary system (Wildman and Parker, 2002). Thus, coexistence is a fundamental assumption in the formulations that follow. However, it must be noted that, in order to compute properties relating to the whole system, individual component properties should be combined. This is presented in later sections.

\subsubsection{Segregation}

Particle mixtures in dynamic systems, such as rotating drums, tend to segregate or separate according to their components. Segregation is due to differences in physical properties of the particles in a given mixture (Jain et al., 2005; Arntz et al., 2008). These differences may be in terms of mass, density or size. Segregation may also be due to the reaction of particles to certain forces acting on the particle bed. In the case of mass-related segregation, the forces include gravitational forces and centrifugal forces. For rotary mills, Agrawala et al. (1997) cited additional causes of segregation, including: lifter configuration, slurry density and flowrate, and ball-to-mill diameter ratio.

There are various segregation mechanisms, some of which are illustrated in Figure 5-1 (Jain et al., 2005). Segregation mechanisms are mainly due to percolation and buoyancy, or a combination of these two. 

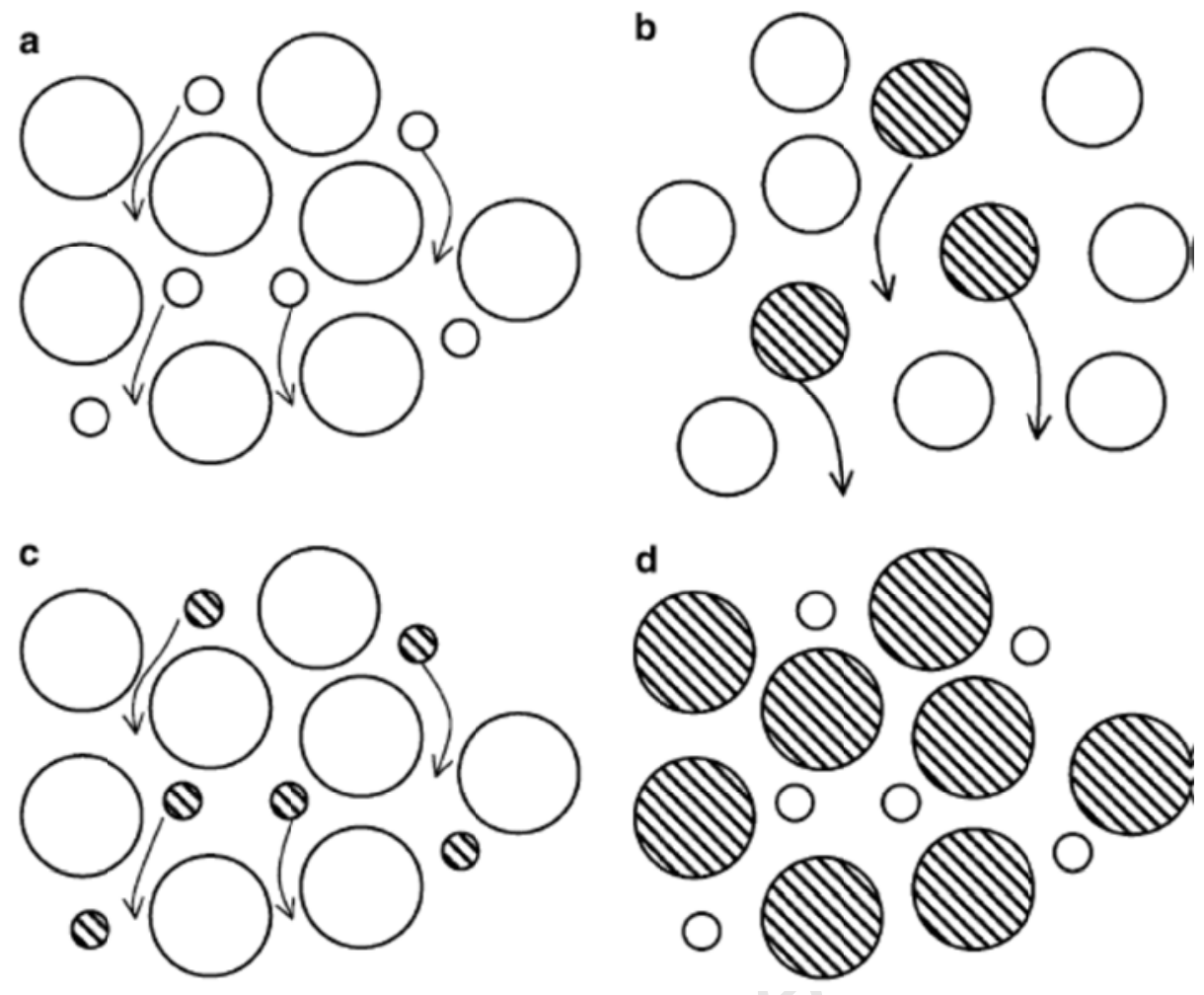

Figure 5-1 Segregation mechanisms: (a) segregation by percolation only; (b) segregation by buoyancy only; (c) segregation when percolation and buoyancy act together; and (d) segregation when percolation and buoyancy oppose each other. Symbol fill: open - lower density particles; filled - higher density particles

Due to segregation, particles of different sizes within a mixture in a rotary system exhibit different packing behaviour. This has been observed in binary systems studied using PEPT (Ding et al., 2001; Fan et al., 2006a). Figure 5-2 shows the occupancy of two tracers of different size in a rotating drum.

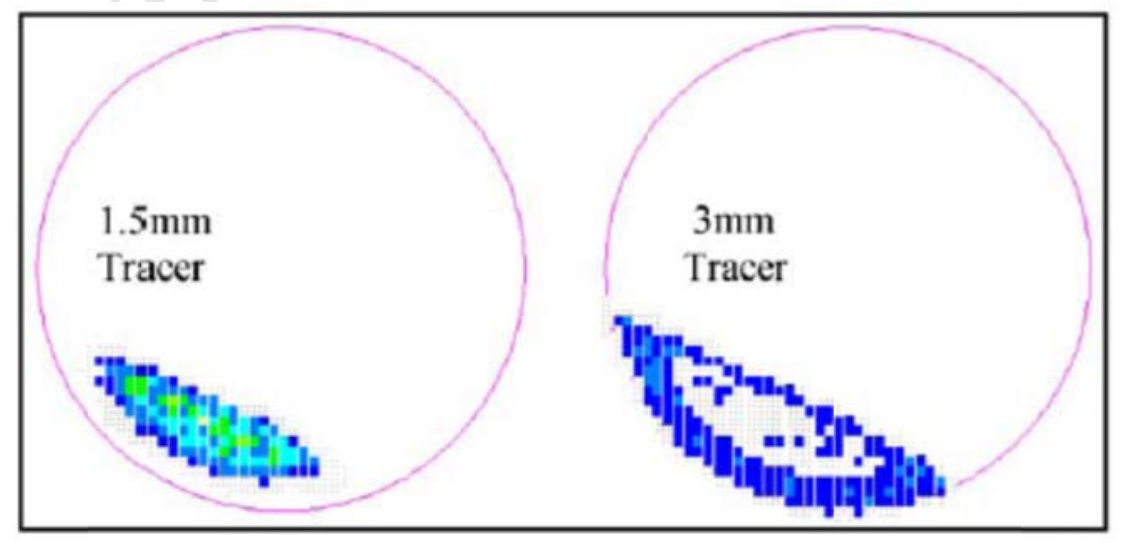

Figure 5-2 Occupancy plot of different sized tracers in a binary system (Fan et al., 2006a)

Figure 5-2 shows that smaller particles tend to pack towards the core of the particle bed, while the larger ones tend to be more concentrated towards the peripheral. However, 
segregation and mixing have been shown to depend on filling and rotation speed (Arntz, et al. 2008). At higher speeds, the arrangement is reversed. The smaller particles tend to be in the peripheral while the larger form the core (Arntz et al., 2008). Arntz et al. suggested that this inverted segregation is as a result of smaller particles percolating through the voids in the expanded bed. The main difference from regular segregation is in the percolation region. In the cataracting flow regime, percolation occurs mainly in the toe region, where the smaller particles sink down quickly towards the drum wall. On the other hand, at lower speeds, percolation takes place across the full length of the flowing layer-or free surface (Arntz et al., 2008).

Ding et al. (2002) also studied the segregation of particles in a rotating drum with a binary filling using PEPT. Figure 5-3 shows the occupancy of small and large particles in a binary system studied using PEPT.
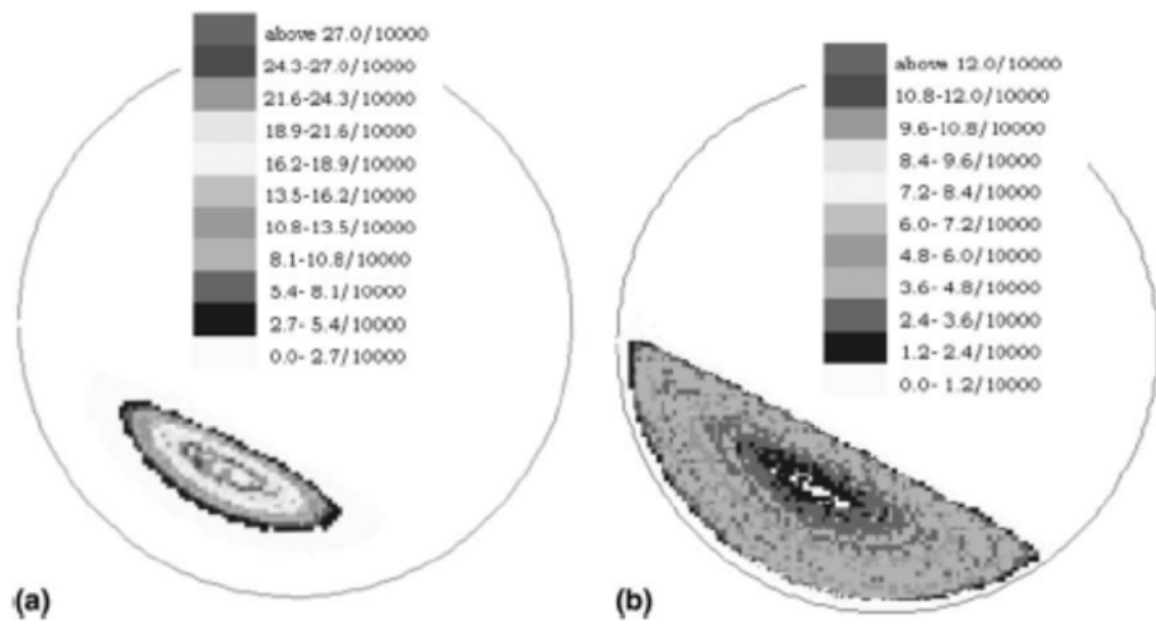

Figure 5-3 Segregation demonstrated by occcupancy of small (a) and large (b) particles (Ding et al., 2002)

From the occupancy plots above, we observe that particles of different sizes exhibit different packing patterns in a multi-component system. The results presented in Figure 5-2 and Figure 5-3 indicate that it is necessary to study different sized particles in a multi-component system in order to understand particle packing behaviour in such systems. They further show that, we cannot rely on results from studies performed on monosize or binary systems.

Segregation of particles different in size is expected to occur under all known flow regimes. This is obvious; especially that different sized particles are still expected to behave differently in the various flow regimes. Furthermore, segregation occurs in both the axial and radial directions. In terms of order, radial segregation occurs before axial segregation occurs 
(Kuo et al., 2006; Rapaport, 2007). However, radial segregation persists even after essentially complete axial segregation (Hill and Kakalios, 1994; Rapaport, 2007). This result implies that in a steady-state system, studying the radial profiles of properties may be sufficient for various purposes. This is not to overlook the various insights we get by studying axial profiles. In this study, axial distribution of porosity is not studied.

\subsubsection{Tracking a particle per size component}

Tracking of different sized particle tracers has been employed in studies of systems which have a particle size distribution—including binary systems (Baumann et al., 1994; Wildman and Parker, 2002). As was discussed earlier, this is justified by the coexistence property (section 5.3.1) and the tendency of particles to segregate (section 5.3.2). In a study by Wildman and Parker (2002), the packing fraction was calculated using the residence time distribution of the two tracers representing the two size components. Figure 5-4 shows the packing fraction profiles for the individual components.
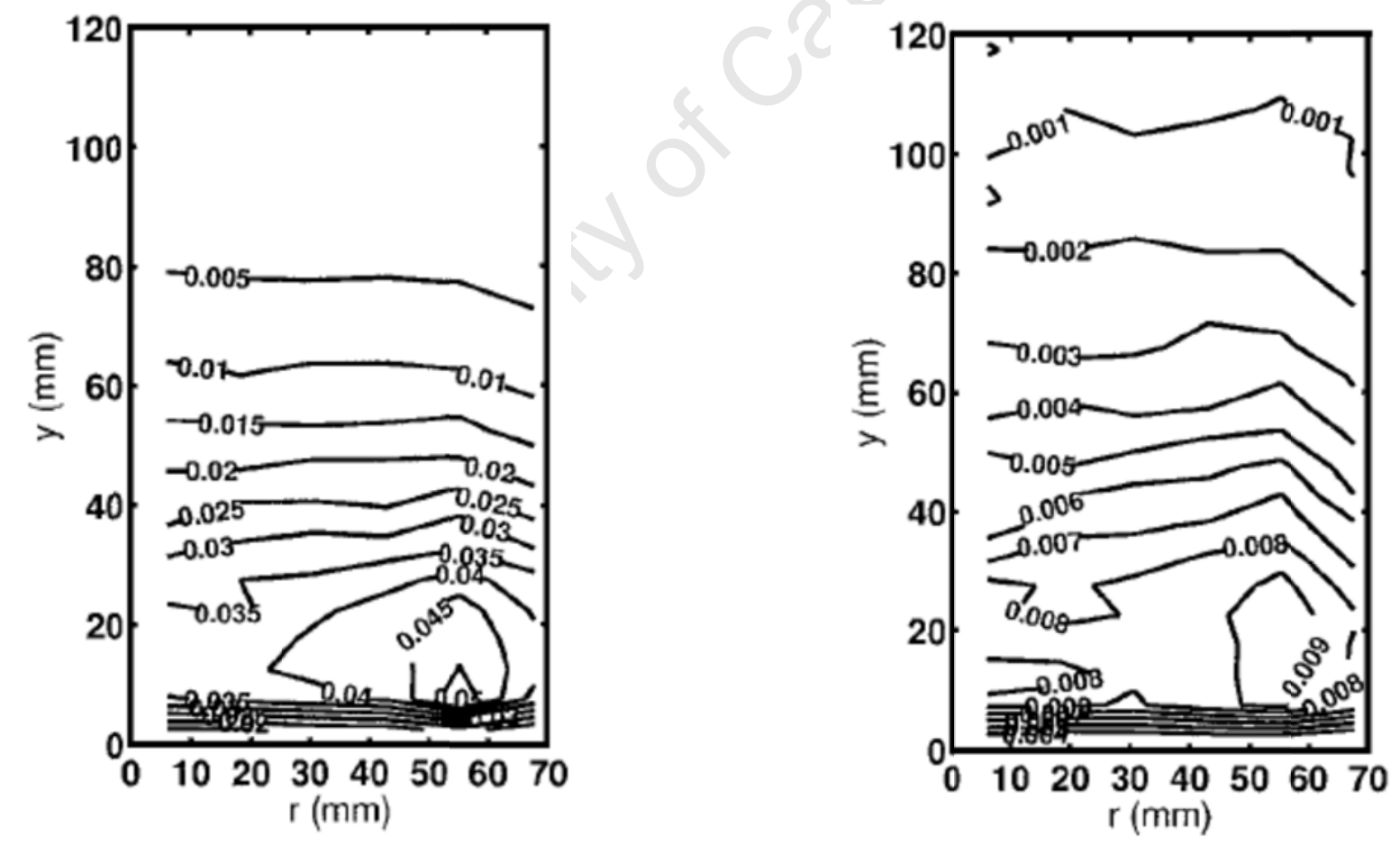

Figure 5-4 Contour maps showing the packing density of different sized particles

Figure 5-4 clearly shows that it is necessary to track particles of different sizes in a multicomponent system. Therefore, in this work, particles of different sizes were tracked. Each of these represented a size component. To justify this further, in terms of coexistence, once 
components segregate, the pattern of segregation is invariant. This has been shown by studies of steady-state segregation (Arntz et al., 2008). This can also be shown by the convergence of centre of mass of individual components (Rapaport, 2001). This convergence is related to the criteria used in this work to determine steady state-power draw. Therefore, each of the tracers tracked effectively represents its size component.

\subsection{Estimating the porosity}

The development of the formulations used for calculating porosity in this work is presented in this section. These formulations are based on the theory presented in the previous sections. For simplicity, a discrete particle size distribution is considered. Thus, there are a fixed number of components, $M$, each with a fixed number of particles. Based on the ergodicity assumption; the residence time is used to estimate the number of particles of each size component in a given region. Thereafter, the solid volume contribution of each component in each region is computed. Ultimately, these volume contributions are combined to give total volume of solids per region. This, in turn, is used to compute packing density, from which porosity is finally calculated.

\subsubsection{Porosity}

The aim of this work was to characterise porosity in terms of spatial distributions. Therefore, the system was divided into volume elements (regions). This was helpful in analysing how the porosity is distributed spatially. Porosity is the fractional volume of voids in a given volume. Thus, if the volume of voids, $V_{v}$, in a given volume element is known, porosity $\varepsilon$ would be given by the following formula:

$$
\varepsilon=\frac{V_{v}}{V_{b}}
$$

Equation 5.10

where $V_{b}$ is the total (bulk) volume of the volume element of interest. The bulk volume includes both the solids and voids. The reader may wish to recall that, porosity, in this case, refers to packing porosity, which is different from the porosity of material.

A related concept is that of packing density or packing fraction. Packing fraction is the ratio of the volume of solids to that of the bulk volume, which is given by the following: 


$$
\eta=\frac{V_{s}}{V_{b}}
$$

Equation 5.11

where $V_{s}$ is the volume of solids in a volume element.

The volume of voids has a very simple relationship with the volume of solids:

$$
V_{v}=V_{b}-V_{s}
$$

Equation 5.12

Thus, porosity can be measured from a known volume of solid, as follows:

$$
\varepsilon=\frac{V_{b}-V_{s}}{V_{b}}
$$

Equation 5.13

Equation 5.13which can be rewritten as follows:

$$
\varepsilon=1-\frac{V_{s}}{V_{b}}
$$

Equation 5.14

Substituting the fraction on the right hand side of the minus sign by left hand side of Equation 5.11 gives:

$$
\varepsilon=1-\eta
$$

Equation 5.15

where $\eta$ is the packing fraction.

Thus, porosity can be computed using a known packing fraction. It is also natural to think that the packing fraction would be easier to calculate from PEPT data. This is because the particle tracer gives information about particles and not about voids. However, as demonstrated above, the voids can be deduced from known information about solids. In particular, if packing fraction is known, then porosity can easily be estimated using Equation 
5.15. In the next section, the use of PEPT data to compute the volume of solids that individual size components contribute to a given volume element is discussed.

\subsubsection{Solids volume computation}

The technique used to compute volume of solids is based on the fact that each tracer represents the packing behaviour of an individual size component. From section 4.2, the proportionality of packing density to the residence time has been used to estimate packing density in monosized and binary systems. In this work, a single tracer's trajectory is used to estimate the probability of locating a particle of that component in a given volume element.

Since the system is divided into volume elements, the immediate goal—at this stage — is to estimate the volume of solids in each element. Then the packing density and porosity can be computed. Consider how one would estimate the volume of solids in a given volume element. If the solids are spheres, the complexity of the problem reduces significantly. If there are $n$ spheres - each with diameter $d$-in a volume element, the volume of solids is given by:

$$
V_{s}=\frac{n}{6} \pi d^{3}
$$

Equation 5.16

Thus, for a known number of particles $n$, the volume of solids can be computed easily. However, for a multi-component system, computing the volume of solids requires knowledge of the number of particles in each size component that are in a given volume element. The computation of these numbers is discussed in the next section.

\subsubsection{Number of particles in a given volume element}

If the number of particles $N_{i}$ in a given size component $i=1,2,3 \ldots M$ were known, and the probability $p_{i j}$ of locating a particle of component $i$ in a volume element $j$ established, the number of particles $N_{i j}$ of $i$ which are in $j$ can be computed. The key question is: how does one determine probability $p_{i j}$ (above) from the trajectory of a single particle? There are two basic ways: firstly, by counting tracer locations in each volume element and then dividing by the total. We shall refer to this method as simple location probability. Secondly, $p_{i j}$ can be determined by estimating the amount of time the tracer spends in each volume element. If residence time is divided by total observation time a residence-time fractional distribution 
results. The third method is a hybrid and gives what is known as time-weighted location probability.

The three methods presented above measure the likelihood that a particle of a given size will be located in a given volume element. This likelihood will be generally referred to as location probability. The fore-mentioned methods can all be used to estimate particle population in each volume element. They only differ in accuracy.

\subsubsection{Simple location probability}

The first step in the simple location probability method involves establishing the number of times $c_{i j}$ a tracer of size component $i$ is located in a given volume element $j$. This count is based on the tracer's trajectory. Dividing $c_{i j}$ by the total number $C_{i}$ of data points on the trajectory gives a simple location probability $L p_{i j}$, given by the following:

$$
L p_{i j}=\frac{c_{i j}}{C_{i}}
$$

Equation 5.17

Despite being used in some PEPT studies of occupancy, the simple location probability is not always a good estimate of location probability. The reason for this is that, due to the location algorithm (section 4.1.3), the time interval between successive locations is not always uniform (Wildman et al., 2000). Thus, the accuracy of this probability measure is subject to the standard deviation in these time intervals.

\subsubsection{Residence-time fractional distribution}

Residence-time fraction is based on the time coordinates associated with each location event on a tracer trajectory. From these time details, the total cumulative time $t_{i j}$ a tracer of component $i$ spends in a volume element $j$ can be computed. Normalising — or dividing each $t_{i j}$ by total observation time $T_{l}$-yields the residence-time fraction $F_{i j}$, given by:

$$
F_{i j}=\frac{t_{i j}}{T_{i}}
$$

Equation 5.18

$T_{i}$ is given by the difference in times between the first and last points on the trajectory. Details of how residence time is calculated are presented later. Residence time seems to be a 
better estimate of the location probability due to the same reason simple location probability is not. Therefore, residence time fractional distribution was the preferred method in this work.

\subsubsection{Time-weighted tracer location probability}

The time-weighted location probability is obtained by weighting the simple location probability by the residence time. Firstly, simple location probability is multiplied by residence time. The result is then normalised to give the time-weighted location probability.

\subsubsection{Total number of particles}

This far, the assumption has been that, the total number of particles $N_{i}$ in each size component $i$, is known. Consider a known residence time fraction $F_{i j}$ of component $i$ in volume element $j$ and number of particles $N_{i} . F_{i j}$ represents the fraction of particles of $i$ that are located in $j$. Therefore, the number of particles $N_{i j}$ of $i$ in $j$ is given by:

$$
N_{i j}=N_{i} F_{i j}
$$

Equation 5.19

Thus, the population of particles of component $i$ in volume element $j$ would be established.

\subsubsection{Solid volume contribution by component $i$ in volume element $j$}

Substituting Equation 5.19 for $n$ in Equation 5.16, the volume of particles of component $i$ in volume element $j$ can be computed as follows:

$$
V_{i j}=\frac{N_{i j}}{6} \pi d_{i}^{3}
$$

Equation 5.20

The next step is to combine the results for all the size components, based on information gathered through tracking different sized tracers. The aim was to compute the total solid volume in each volume element.

\subsubsection{Combining the volumes of solid from different size components}

An important aspect of this work was the method of weighting solid volumes of individual components when computing the total volume of solids. 
The formulation is based on the coexistence assumption, size segregation (consistent at steady state - see section 5.3.2), and the additive property of the volume of solids. Coexistence and segregation also imply that, on time-average, particles do not overlap. Thus, if the system is studied for a sufficiently long period, the volume of solids for each component adds to the total volume of solids in a simple way. Simple here means without additional weighting, besides that provided by the size distribution, implicitly. The total volume of solids is, therefore, a sum of volumes of solids contributed by each component (section 5.4.3.5).

The combination proposed above can be demonstrated by a simple system (Figure 5-5, below) of differently sized spheres in one volume element. For clarity the system is presented in two dimensions, where circles represent spheres.

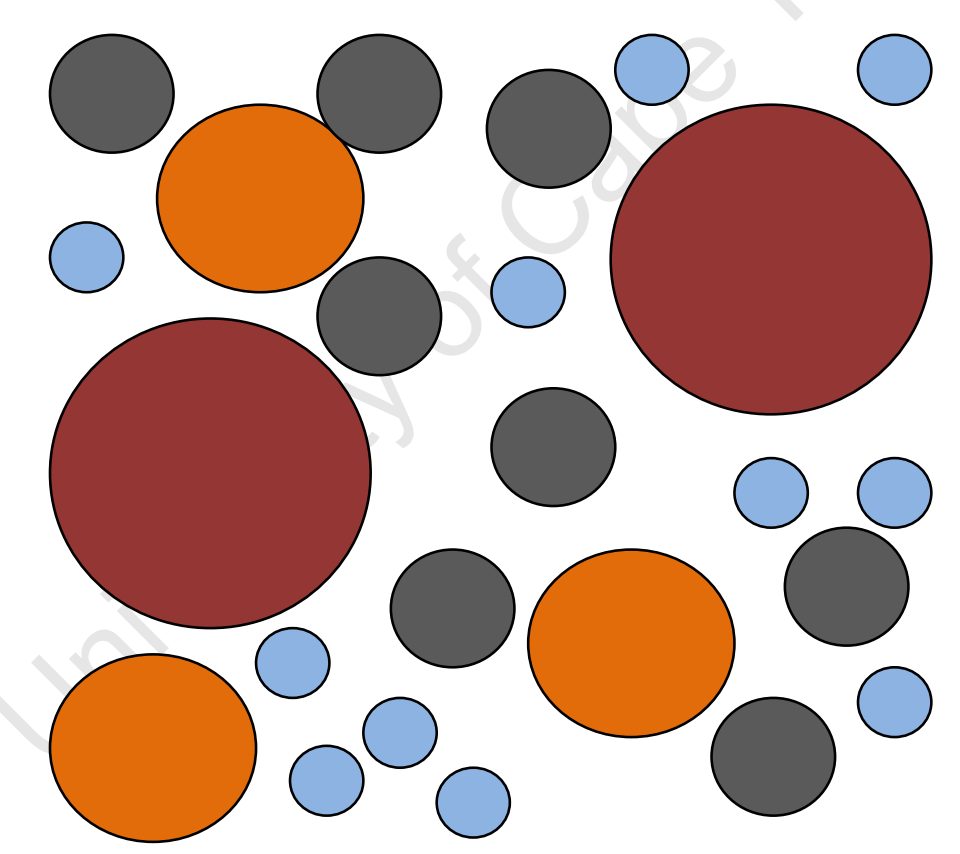

Figure 5-5 Schematic of particles in a multi-component mixture in a volume element

From Figure 5-5, it is shown that each component contributes to the total volume of solids in the volume element in a natural way. Thus, the total volume of solids in the system above is the sum of volumes of all the particles-grouped into components by different colours. Figure 5-5 also demonstrates that the particles presented therein can only take up unique positions and there is no overlapping. 
The additive and coexistence property can also be justified by the studies that have been done using numerical techniques. In Figure 5-6 (Dury and Ristow, 1999) is shown a plot of packing density of individual components, and that of the mixture of the two components.

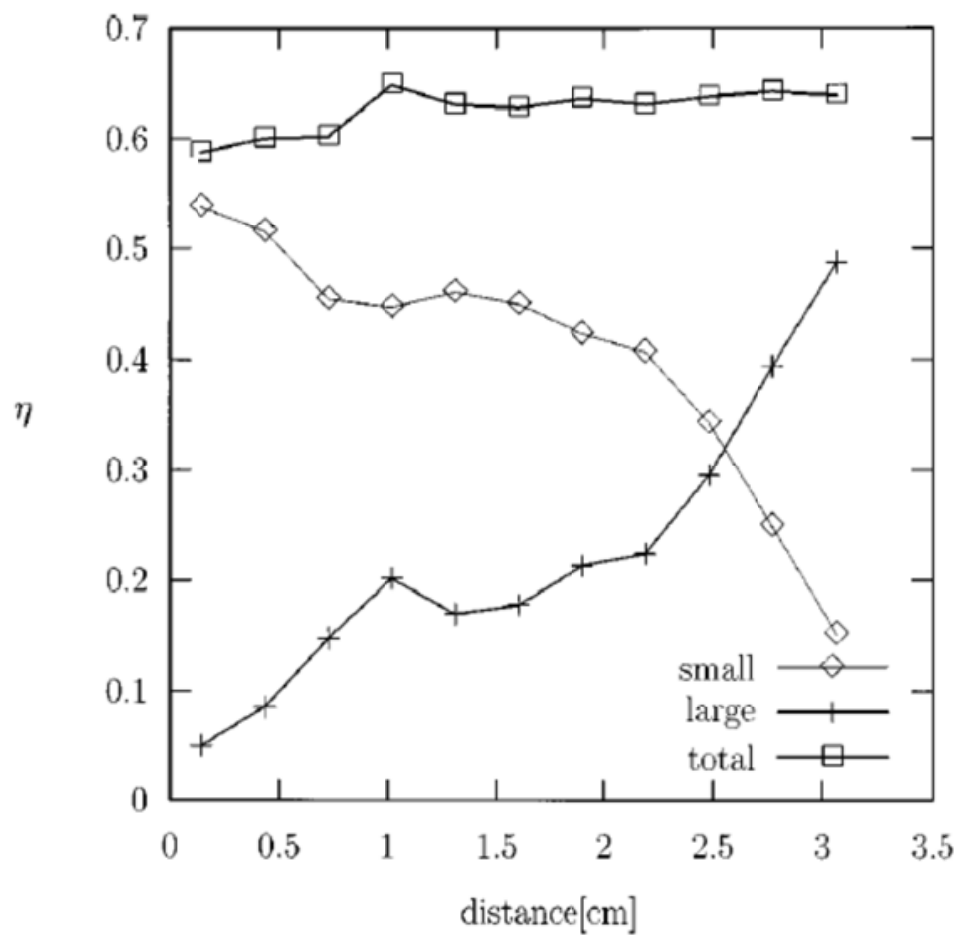

Figure 5-6 Volume fraction of small and large particles in a cylinder for a volume filling fraction of $66.4 \%$

From Figure 5-6, it is shown that the packing density of the mixture (top most line) is the sum of the packing densities of the two components. Thus, is can be observed that the volume of solids of individual components adds up to the total volume of solids in the mixture. This is because the packing densities of individual components have a common denominator $V_{b}$, as shown earlier in Equation 5.11.

Thus, the total volume of solids in a given volume element $j$ is given by the sum of the individual component solid volume contributions (Equation 5.20). This is given by:

$$
V_{j}=\sum_{i=1}^{M} V_{i j}
$$

Equation 5.21

where $M$ is the number of size components.

Equation 5.21 can be rewritten as follows based on Equation 5.19 and Equation 5.20: 


$$
V_{j}=\frac{\pi}{6} \sum_{i=1}^{M} N_{i} F_{i j} d_{i}^{3}
$$

Equation 5.22

\subsubsection{Porosity calculation}

Since the formula for computing the volume of solids in a given volume element has been established, the porosity can now be computed. Firstly, the packing density of a given volume element $j$ can be computed using the solid volume $V_{j}$ (Equation 5.22). Thus, by substituting Equation 5.22 into Equation 5.11, packing density is given by:

$$
\eta_{j}=\frac{\pi}{6 V_{b j}} \sum_{i=1}^{M} N_{i} F_{i j} d_{i}^{3}
$$

Equation 5.23

where $V_{b j}$ is the bulk volume of volume element $j$.

Substituting Equation 5.23 into Equation 5.13, porosity is given by the equation:

$$
\varepsilon_{j}=\frac{V_{b j}-\frac{\pi}{6} \sum_{i=1}^{M} N_{i} F_{i j} d_{i}^{3}}{V_{b j}}
$$

Equation 5.24

Equation 5.24 can be reduced to the form of Equation 5.15, as follows:

$$
\varepsilon_{j}=1-\frac{\pi}{6 V_{b j}} \sum_{i=1}^{M} N_{i} F_{i j} d_{i}^{3}
$$

Equation 5.25

Thus, the formula for computing porosity of multi-component mixtures using PEPT data has been established. This formulation is used in all porosity calculations in subsequent chapters.

\subsubsection{Other issues}

Other issues of consideration included: determining how many particles to consider as part of the whole system. This was due to the fact that some particles would cataract-or be inflight - and not part of the bulk charge, at high speeds. This turned out to be a trivial problem. 
As will be observed later, the formulation manages this naturally, without having to worry about the particles that are not part of bulk charge. The reason for this is that, the regions of free flight are also included during volume element segmentation. Thus, particles in these regions are accounted for by the part of the tracer's trajectory that passes through these regions. In addition, the number of particles and the volume of solids for this region are also computed. These are also assigned to their respective volume elements, based on computed location probability. However, as would be expected, the probability that particles will be located in these volume elements. Hence, the packing fraction of these regions is negligible. Thus, the formulation handles the cataracting phenomenon implicitly.

\subsection{Conclusion}

In this chapter, the mathematical formulations used for computing the porosity of multicomponent mixtures in rotary mills using the PEPT technique have been developed. These formulations are based on three assumptions: 1) ergodicity; 2) coexistence; and 3) steady state. The proposed method to study a particle mixture is by tracking a particle from each size component. Each such tracer particle is expected to represent its size component. Therefore, time averaged properties of the tracer can be used to determine properties of a given size component. Consequently, the fractional residence time of each tracer would be used to estimate the location probability of each respective component.

The formulation applied fractional residence time to computing the volume of solids contributed by each component in a given volume element. Thereafter, the total volume would be computed and packing density calculated, per volume element. The porosity can then be calculated from the known packing density. This would allow for the modelling of porosity, as a function of geometric parameters, in the form of a spatial distribution.

The techniques developed in this chapter can also be used on any system where only limited particle tracking data is available — not just PEPT. The main modification required would be on the method of estimating location probability. The next chapter discusses computational aspects related to the formulations presented is this chapter. 


\section{Computational aspects}

Having established formulations for computing porosity in multi-component mixtures, it was important to consider the computational framework within which these formulations would be implemented. From the previous chapter, the following are some of the computational aspects that need to be established: programming framework, determination of volume elements size, number of particles in each component, and location probability algorithm.

\subsection{Programming framework}

The programming language used in this work is MATLAB. However, the strategies presented below are independent of the programming language used. The choice of MATLAB was motivated by its rich matrix libraries, which made programming less tedious. Thus, all strategies can be implemented in any programming language that provides the basic structures required for similar computations. The key issue discussed in this chapter is the computation of residence time fractional distributions.

\subsection{Number of particles in a size component}

In a PEPT experiment, the number of particles in the system can be quite challenging to determine, especially if breakage exists. However, if breakage is absent and the mixture is of a known size distribution and quantity, the number of particles can be determined easily. In this work, it was assumed that there was no breakage in the system. Further, the particle size distribution was discrete.

The particle size distribution was based on the mass of particles. Therefore, the mass of particles in each size component was known beforehand. In addition, the density of the particles was also known - the density of glass beads: $\sim 2700 \mathrm{kgm}^{-3}$. For given mass $m$ and density $\rho$, volume $V$ can be computed, using the mass-density-volume relationship, as follows:

$$
m=\rho V
$$

Equation 6.1

From Equation 6.1, the total solid volume $V_{i}$ for each size component $i$ can be computed, since density and mass are known. It must be noted that $V_{i}$ does not include voids between 
particles. Therefore, if the volume of one particle $V_{p i}$ is known, the number of particles, $N_{i}$, can be determined. This can be achieved by dividing $V_{i}$ by $V_{p i}$, as follows:

$$
N_{i}=\frac{V_{i}}{V_{p i}}
$$

Equation 6.2

Thus, the number of particles needed for computing porosity could be determined easily.

\subsection{Volume element design}

To characterise the spatial distribution of porosity, the system must be divided into smaller volume elements. However, this study only considered the transverse section of the mill. The volume elements were rectangular and had the same size — length and breadth — as shown in Figure 6-1.

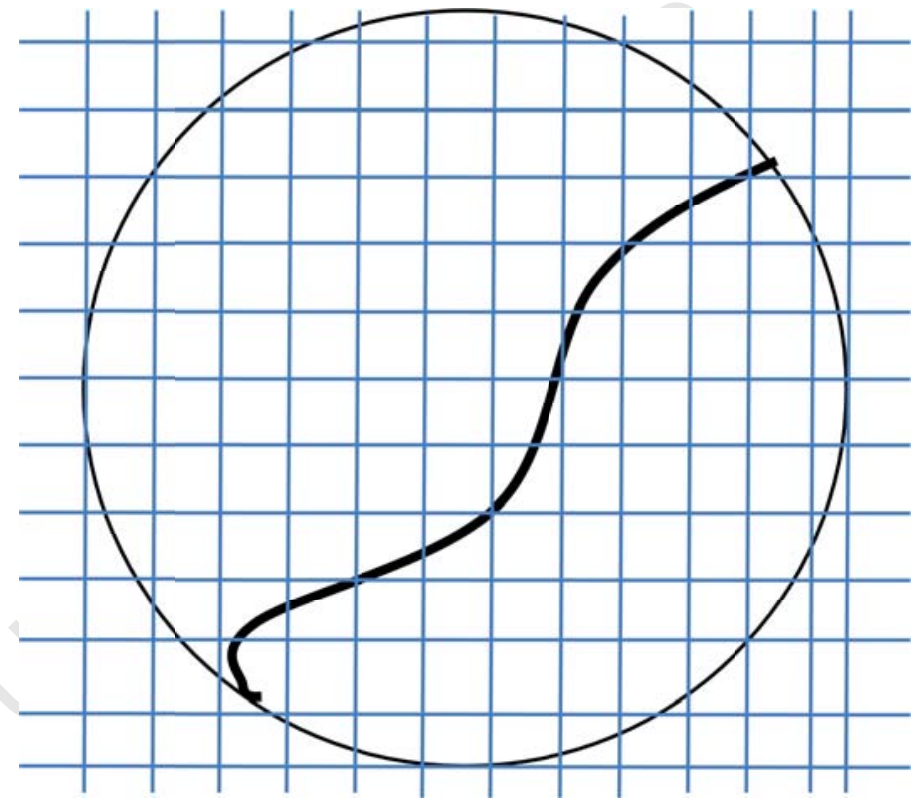

Figure 6-1 Transverse view of volume elements

The volume of the segments shown above is computed by multiplying the area of the rectangles in the two dimensional view by relevant mill length. Essentially, the volume elements are cuboids. The relevant mill length is the inner axial length of the mill occupied by the charge. This excludes the pulp chamber. The residence time and porosity calculations are all based on these volume elements. 
The size of volume elements depends on the method used for estimating location probability. This also depends on the size of the mill. The first criterion is that there must be enough volume elements to give a meaningful size distribution. The second is that the elements must be big enough to give accurate location probability. It is quite obvious, that if simple location probability is used, the elements must be a number of diameters long and wide-about two or three - of the largest particles. If, however, fractional residence time is used, the volume elements can be much smaller. This is because, the effect of the tracer being located on the border of two or more (up to four) volume elements is handled by the residence time algorithm (as will be observed in the next section).

Another reason the volume elements must be small enough is to reduce the effect of the mill shell on the volume calculations of the elements along that region. If the volume elements are very small, the effect of the mill shell can be ignored. It seems, therefore, that with a small mill, smaller volume elements are more attractive. However, if the charge consists of large particles, large volume elements would be desirable. Thus, for a smaller mill, residence time fractional distribution is a more convenient method to use in handling such conflicting objectives.

Finally, to estimate the radial-angular porosity profiles, it is natural to work in polar coordinates, especially with the cylindrical nature of the system. Thus, it was inevitable to work with polar coordinates to perform such computations.

\subsection{Computation of residence time fraction}

The computation of residence time fraction is discussed in this section. The key question is how residence time actually gets assigned to volume elements.

\subsubsection{Time allocation algorithm}

A simple algorithm for estimating the residence time for each volume element starts by finding the volume element in which the first tracer location occurs. Once this initial volume element is established, the algorithm advances to the next location. It then checks if that location lies within the same volume element. If it does, the time difference between the two locations is assigned to the current volume element. This increases the residence time of the volume element by the time difference.

If, however, the tracer moves into another volume element, that volume element becomes current. In that case, the time difference has to be shared between or among the volume 
elements through which the tracer is expected to have passed between successive locations. Thereafter, the algorithm continues on to find the next location's volume element. This process continues until the location has been considered. How the time difference is shared among volume elements is an important problem.

\subsubsection{Time-difference allocation to volume elements}

There are a number of possible cases whenever a tracer's consecutive locations lie in different volume elements. The first is where the tracer moves in one dimension. This implies that the edges of the affected volume elements have the same $\mathrm{x}$-coordinates or $\mathrm{y}$-coordinates, depending on the affected dimension. The second is where these volume elements differ in both $\mathrm{x}$ and $\mathrm{y}$ dimensions. In other words, the tracer is seen to move diagonally. These cases are discussed separately.

\subsubsection{One-dimensional variation in consecutive volume elements}

Whenever the tracer moves between volume elements in one dimension, a straight line can be used to effectively estimate the path of the tracer between two successive locations. Thus, line $L_{i}$ can be used to connect two points $P_{i}$ and $P_{i+1}$. This is based on the assumption that, if the points only differ in one dimension, then curvature of the curve linking them is not very significant. Thus, a curve can be replaced by a straight line.

The proportions in which the affected bins share the time difference is given by the distances $d_{j}$ the tracer covers in each of the affected volume elements. This is illustrated in Figure 6-2.
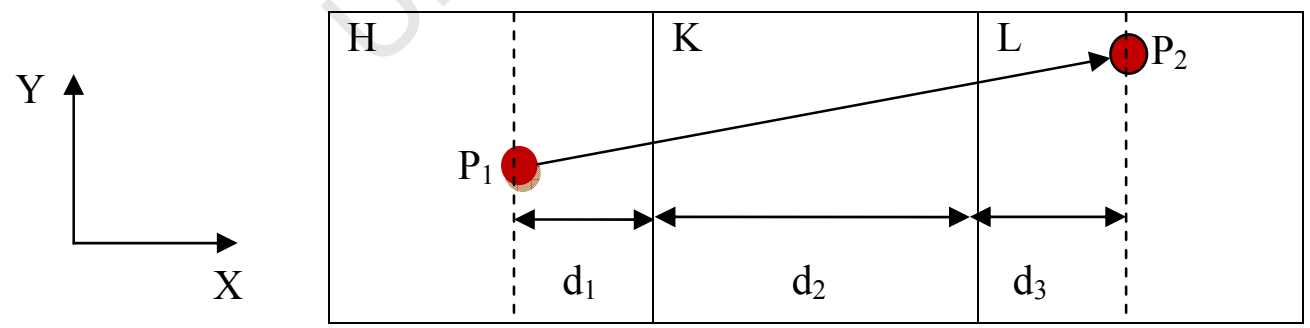

Figure 6-2 Diagram showing the allocation of time based on distance $d_{j}$ the tracer covers in a given element using a straight as a path estimator

Note that each distance $d_{j}$ in Figure 6-2 was measured in one dimension instead of computing the distance of the lines segment. Accordingly, the distances are either parallel or 
perpendicular to any one axis - either the x-axis or y-axis. The justification for this is given graphically based on similarity of triangles, shown in Figure 6-3.

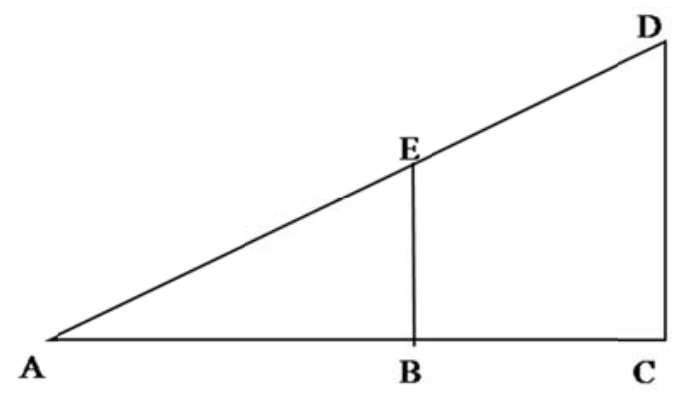

Figure 6-3 Similar triangles with ratios given as follows $\mathrm{AB} / \mathrm{AC}=\mathrm{AE} / \mathrm{AD}=\mathrm{BE} / \mathrm{CD}$

From Figure 6-3, there is no difference in the proportion of distances: whether diagonal or perpendicular; provided there is consistency in any given case. Therefore, the distance $d_{l}$ in Figure 6-2 is computed by subtracting the x-coordinate of location $P_{1}$ from that of the right edge of the volume element H. $d_{2}$ is defined as the perpendicular distance between the left and right edges on the volume element $\mathrm{K} . d_{3}$ is given by the difference between the $\mathrm{x}$ coordinate of location $P_{2}$ and that of the left edge of volume element L. Computationally, this approach is far cheaper than using distances along the line $L_{i}$.

From Figure 6-2, the fraction of the time difference $t_{\text {diff }}$ between $P_{1}$ and $P_{2}$ that the tracer spends in the left most volume element, $\mathrm{H}$, is given by the following:

$$
t_{1}=\frac{d_{1}}{d_{1}+d_{2}+d_{3}} t_{d i f f}
$$

Equation 6.3

Generally, the fraction of $t_{\text {diff }}$ allocated to volume element $j$ of $Q$ affected volume elements, can be computed using the following equation:

$$
t_{j}=\frac{d_{j} \cdot t_{d i f f}}{\sum_{i=1}^{Q} d_{i}}
$$

Equation 6.4

This formula is based on an assumption that the tracer speed does not change significantly, between the two locations. This assumption is considered sufficient, because of the short distances travelled by the tracer in these cases. Thus, any change in speed can be ignored. 


\subsubsection{Two-dimensional variation in consecutive volume elements using a linear estimator}

The next case considered is when the tracer traverses a number of volume elements diagonally. Figure 6-4 shows a linear approximation of the path connecting two consecutive points $P_{3}$ and $P_{4}$.

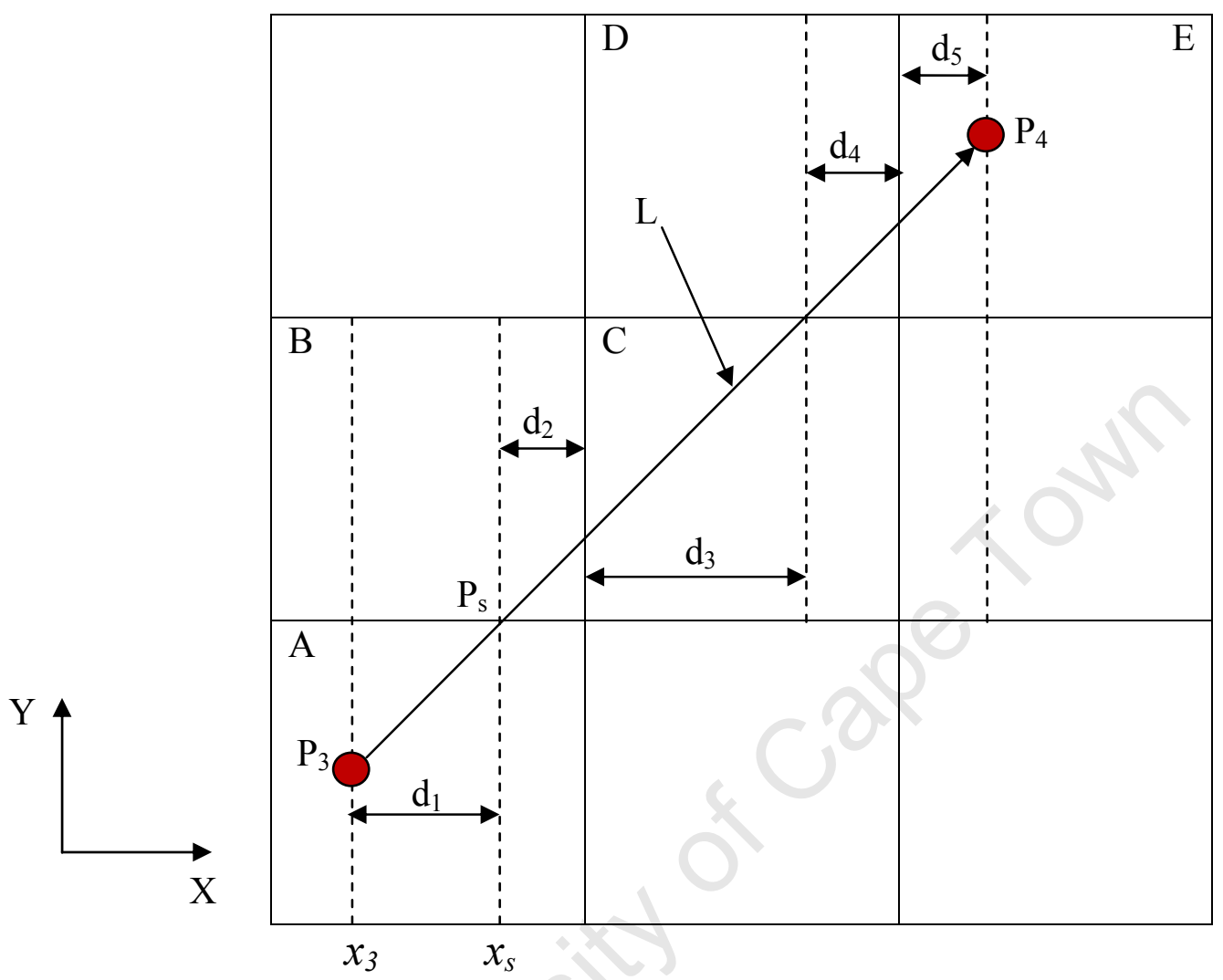

Figure 6-4 Straight line estimate of the path a particle takes across volume elements

In the above case, where the tracer moves diagonally, the fraction of the time $t_{\text {diff }}$ it spends in the affected volume elements is determined by the distance it covers in each volume element. Once again, the path of the tracer can be estimated using a straight line L. In this case, the fractional distance for each affected volume element can be established using either the $\mathrm{X}$ or $\mathrm{Y}$ components of the displacement in respective volume elements. Whichever component is used should be used consistently. Thus, from Figure 6-4, the distances $d_{1}$ to $d_{5}$ represent the fractional distances covered by the tracer in volume elements A to E, respectively. In this case, the X components of displacement are used. The next problem would be to determine the distances, numerically.

\section{Determining the horizontal distances in the two-dimensional case}

From Figure 6-4, the first is $d_{1} . d_{1}$ is the difference between the $\mathrm{x}$-coordinate of the point $\mathrm{P}_{3}$ and that of the point of intersection $\mathrm{P}_{\mathrm{s}}$ of the line $\mathrm{L}$ and top edge of the volume element $\mathrm{A}$. 
Thus, one needs to find the point of intersection between the two lines referred to above. The equation of the line $\mathrm{L}$ is easily established by considering the end points $\mathrm{P}_{3}\left(x_{3}, y_{3}\right)$ and $\mathrm{P}_{4}\left(x_{4}\right.$, $\left.y_{4}\right)$, as follows:

$$
y-y_{3}=m\left(x-x_{3}\right)
$$

\section{Equation 6.5}

where $m$ is the gradient, given by:

$$
m=\frac{y_{3}-y_{4}}{x_{3}-x_{4}}
$$

Equation 6.6

The edges of the volume elements have simple one-dimensional equations. These are established when dividing the system into volume elements. These are of the form $x=a$, for the vertical edges and $y=b$ for the horizontal edges.

If the equation of the top edge of volume element $\mathrm{A}$ is $y=c$, then the $\mathrm{x}$-coordinate $x_{s}$ of $\mathrm{P}_{\mathrm{s}}$ can be computed by substituting $c$ for $y$ in the equation of the line L. Therefore, $x_{s}$ is given by

$$
x_{s}=\frac{c-y_{3}+m x_{3}}{m}
$$

$$
\text { Equation } 6.7
$$

Thus, $d_{1}=\left|x_{3}-x_{s}\right|$.

In Figure 6-4, $d_{2}$ represents the distance in the $\mathrm{x}$-direction that the tracer covers in volume element B. $d_{2}$ is the difference between $x_{s}$ and the x-coordinate of the right edge of volume element B. This edge is vertical and has equation in the form $x=h$. Thus, the distance $d_{2}$ would be given by $d_{2}=\left|h-x_{s}\right|$. The process continues until $d_{5}$ has been determined. The fraction of the $t_{\text {diff }}$ between $\mathrm{P}_{3}$ and $\mathrm{P}_{4}$ that the tracer spends in each of the volume elements $\mathrm{A}$ to $\mathrm{E}$ is given by Equation 6.4.

\subsubsection{Two-dimensional variation in consecutive volume elements using a polynomial estimator}

A more accurate method of sharing the time difference between successive locations is to approximate the actual path of the tracer. Unfortunately, the particle dynamics in the mill 
makes this very difficult, if not impossible, especially in the toe region, where motion is chaotic. Fitting a curve to data points in that region would probably not yield any meaningful result. It actually has the potential of making the data more inaccurate. Accordingly, a straight line would be sufficient estimate in the absence of enough knowledge of the trajectory. Thus, the linear estimator method was used in this work for time difference allocation between successive locations. In summary, the reasons for settling for this method include the following:

1. The differences between successive trace locations are not very large. Furthermore, for very short distances, the difference between a curve and a line is very minimal. In fact, in a computational sense, curves are made up of short straight lines;

2. The volume elements are not very small, compared with the distance between consecutive locations. Therefore, the benefits of using curves, over lines, are very minimal. This is demonstrated in Figure 6-5.

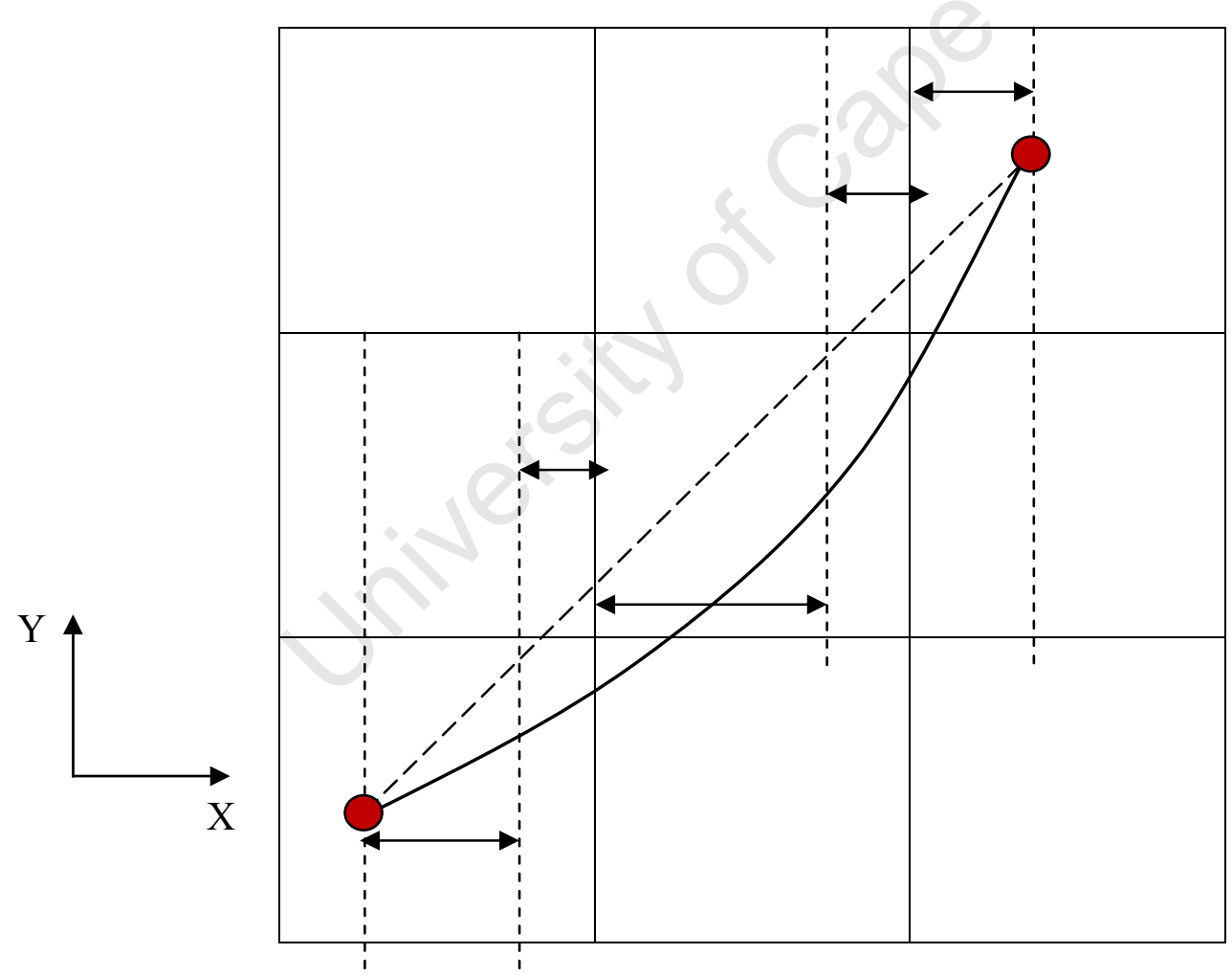

Figure 6-5 Comparing time sharing methods: line and curve

Figure 6-5 shows a curve with hypothetical curvature. If a curve were fitted to the data as shown above, the time allocation may differ only slightly. But as seen from the path of the curve and that of the line, the curve passes through three volume elements. But it 
should be noted that the distance lost by other elements that had the line passing through them in Figure 6-5 is minimal.

3. Therefore, considering the fact that we cannot fit curves in the mill environment with certainty, the differences can actually be ignored;

4. In addition, the spatial distribution would not be significantly affected because the cells are adjacent. Thus, using straight lines as tracer path estimators is sufficient, at least for the purpose of this study.

However, the best fit through the data is one that satisfies smoothness and continuity of derivatives (like the standard schemes for spline fitting). Straight line fitting also has the potential to introduce artificial residence time allocations in certain regions while missing it in others. For the purposes of this thesis, the straight line fitting method is chosen with an understanding that it has limitations.

\subsubsection{Final considerations on residence time algorithm}

Considering volume element size; if residence time is used as an approximation of location probability, particle size matters less than it would, if simple location probability was used. The latter requires volume elements that are large enough to accommodate two or three particle diameters of the largest particles. In the case of residence time, this need not be the case. If a particle, for example, is located between two volume elements, the time sharing algorithm shares the particle location between these elements, through the fractional residence time. This would not be possible if simple location probability were used. In this case, the location would have to be counted as being only in one of the volume elements. Thus, for residence time, the volume elements can be made small enough to ensure finer spatial distributions.

Finally, once the algorithm has traversed the whole data set, the residence time of each volume element represents the amount of time the tracer spends there. Therefore, the fractional residence time is found by dividing the residence time of each volume element by the total observation time $T . T$ is the sum of residence times of all volume elements. This approach, results in residence time distribution of a particular tracer across the mill's transverse section. This can then be used to compute the porosity of the charge and its distribution, as discussed earlier. 


\subsection{Conclusion}

In this chapter, computational aspects of this work have been discussed. These relate to salient issues about the implementation of mathematical formulations presented in the previous chapter. In particular, a method for determining the number of particles per size component was established. Aspects of volume element size were also discussed. Finally, an algorithm for estimating residence time fractional distributions was developed. The next chapter discusses experimental aspects of this work. 


\section{Experimental Setup}

This chapter discusses the experimental programme for this work. The experimental design is based on the theoretical formulations presented in chapters 4,5 and 6 . The key design issues include: equipment design, materials used, and parameters considered. The method which was used to measure the dynamics of particles in the rotary mill is PEPT. The experiments were conducted at the Positron Imaging Centre at the University of Birmingham, United Kingdom. The data collected from these experiments was converted into tracer particle position data using techniques presented in section 4.1.3. The data files that result from this conversion were then used to compute porosity using the techniques presented in the previous two chapters. The results are presented in the next chapter.

\subsection{The laboratory scale mill}

As was discussed in section 2.1.2.1, rotary mills are very large equipment. However, due to equipment size limitations on the PEPT facility, only a laboratory scale tumbling mill could be studied. The mill that was used in this study is similar to the real tumbling mill in its key features. The main differences are that it is smaller and made of different material. A schematic image of the mill is shown in Figure 7-1.

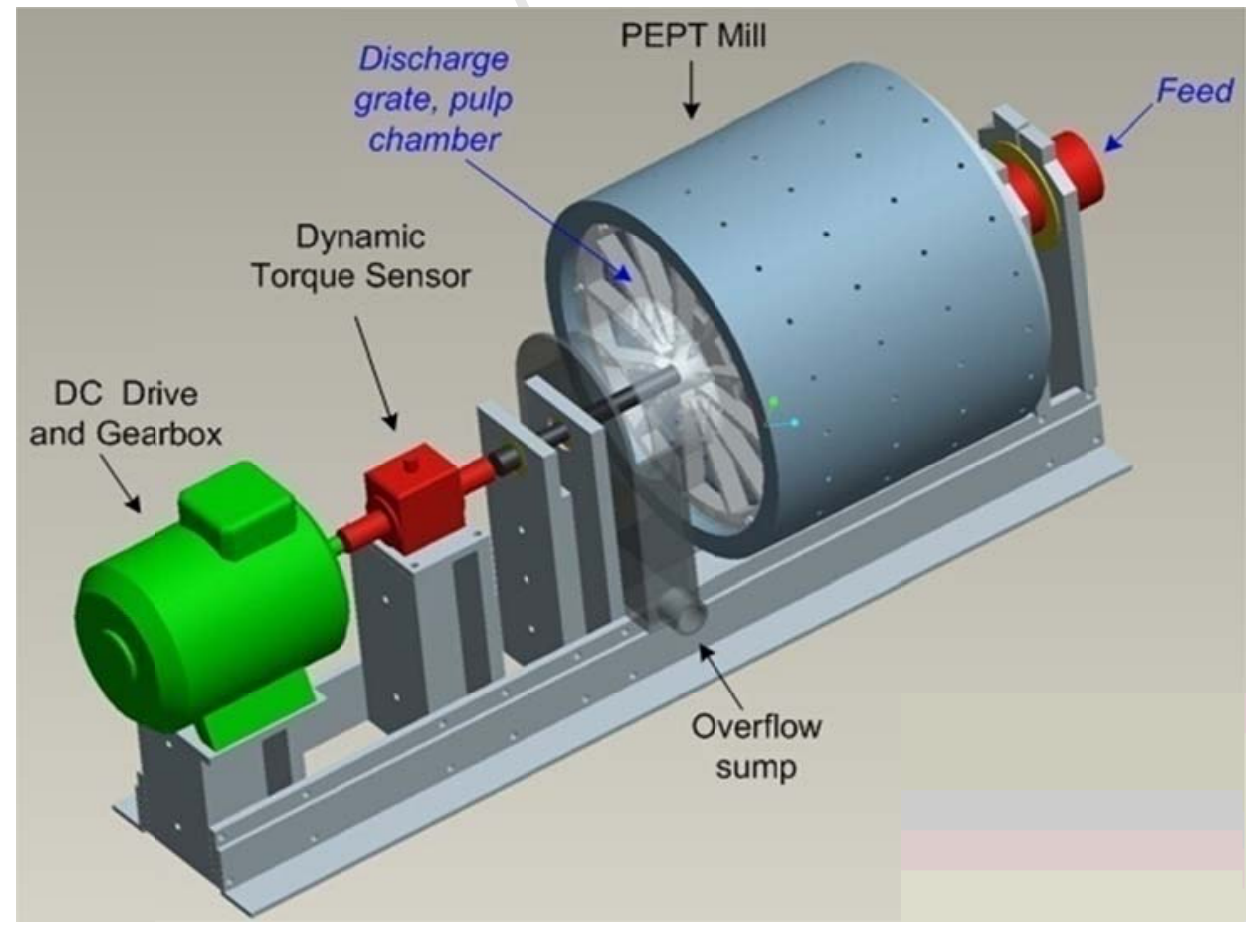

Figure 7-1 Schematic experimental mill used in the experiments 
Figure 7-1 shows the major components of the experimental mill. The mill had a feed end, through which the particles were fed into the mill. On the opposite end are the discharge grate, the pulp chamber and the discharge trunnion. The mill shell, pulp lifters and lifters were made of High Density Polyethylene (HDPE), with a specific density of $\sim 0.95$. The grate and the mill circular end covers were made of clear acrylic. Attached to the mill-via an axle - was a DC drive with a step-down gear box, a torque sensor and a reticulate pump (not shown in Figure 7-1). The DC drive is what would rotate the mill, when in operation. The torque sensor was used to measure the dynamic fluctuation of the mill load.

\subsubsection{Mill dimensions}

The mill had an internal length, including the pulp chamber, of $300 \mathrm{~mm}$ and internal diameter of $300 \mathrm{~mm}$. The length of the pulp chamber was about $30 \mathrm{~mm}$. This implies an internal mill length, excluding the pulp chamber, of about $270 \mathrm{~mm}$. This $270 \mathrm{~mm}$ region contained the charge for which porosity had to be studied.

\subsubsection{Lifters}

In order to depict real tumbling mill mechanics, the laboratory mill had lifters. Figure 7-2 is an image showing an internal view of the mill, with the 20 lifters along the mill shell.

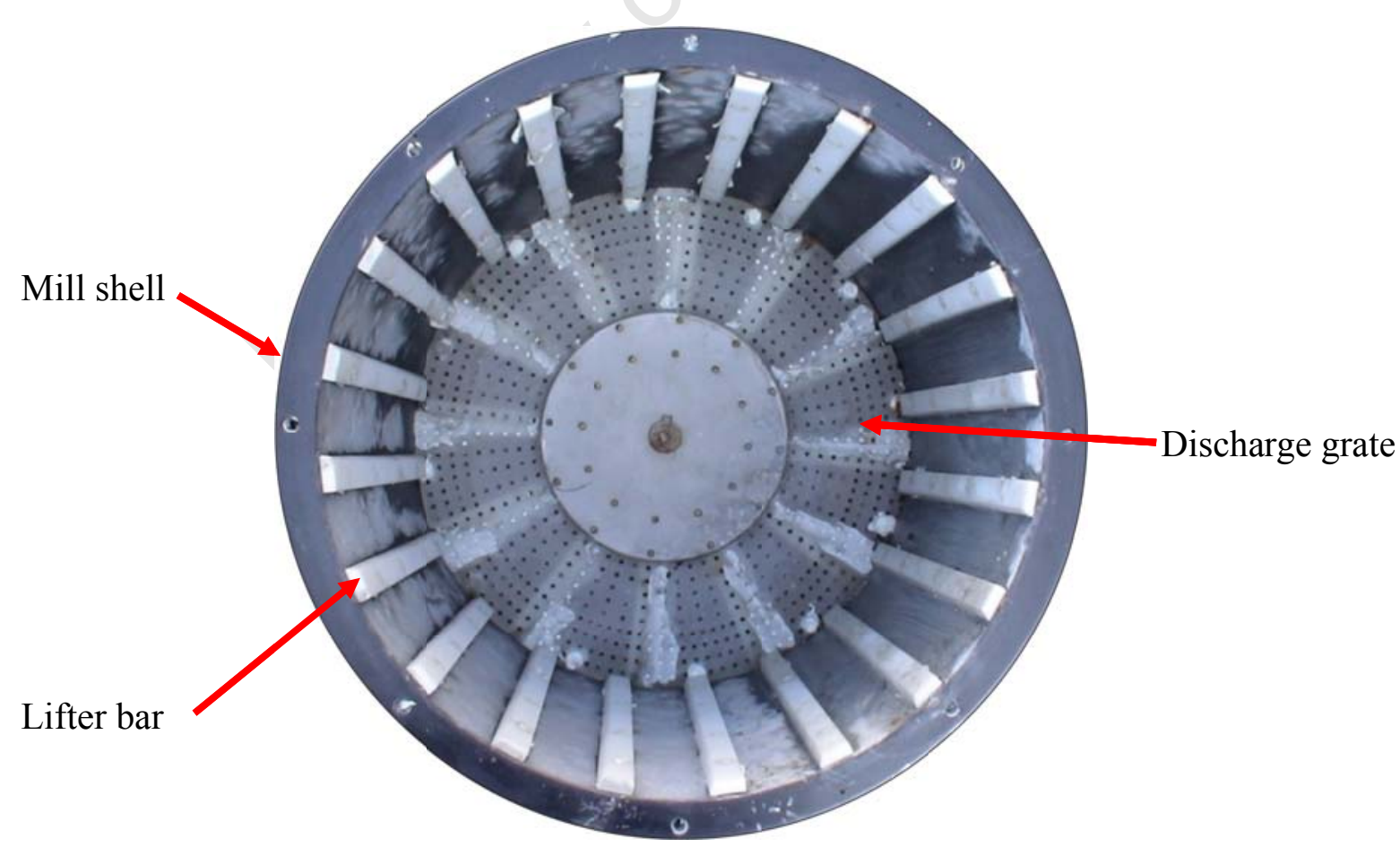

Figure 7-2 Internal view of the mill

In addition, Figure 7-3 shows a schematic of the lifter geometry described above. 


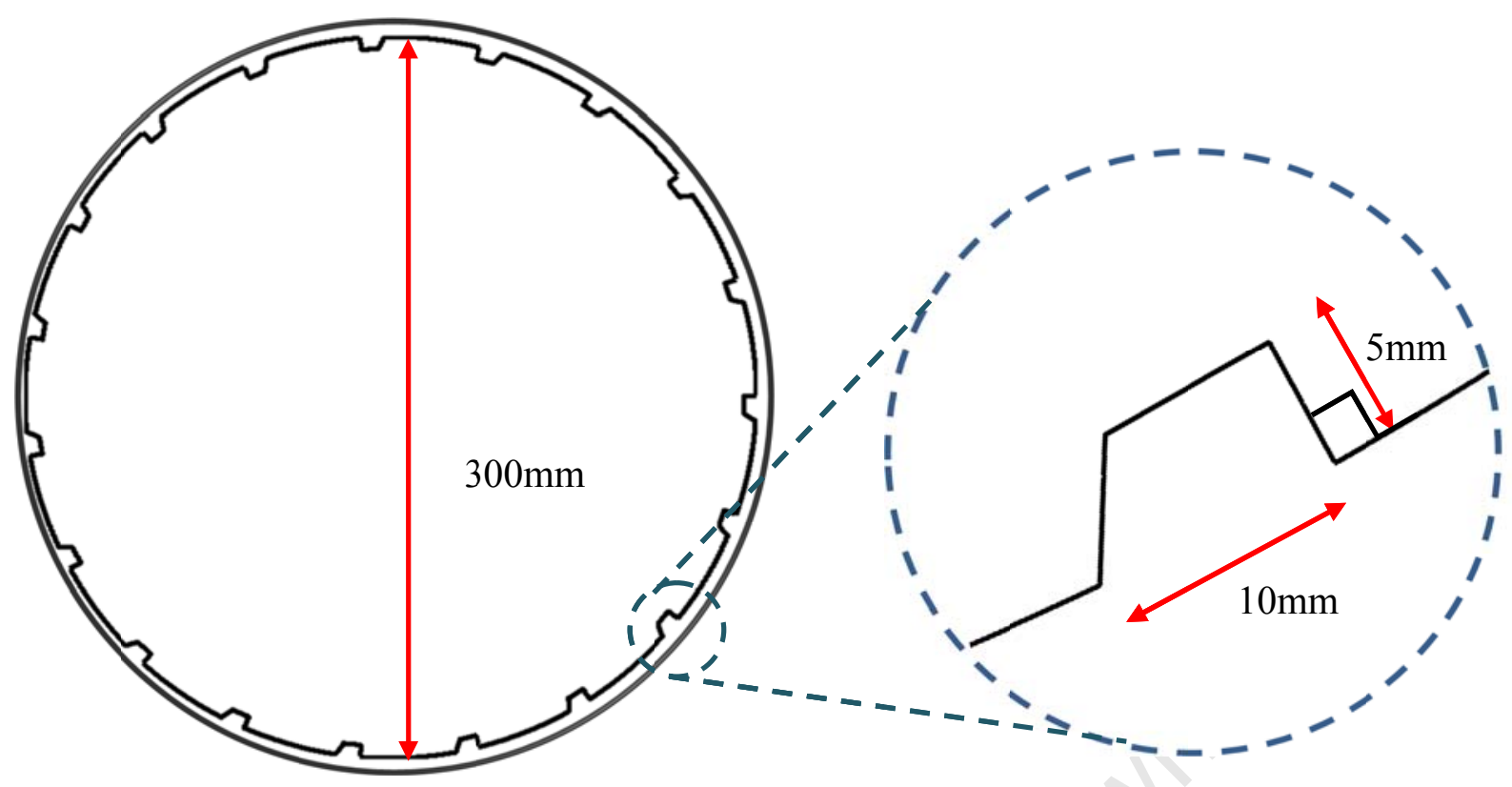

Figure 7-3 Schematic of mill shell outline and magnified view of single lifter

The lifters had a base wide of $10 \mathrm{~mm}$ and a height of $5 \mathrm{~mm}$ each. The lifter face forms a right angle with the mill shell. For this work, only one lifter design was studied.

\subsubsection{Grate design}

The mill had a discharge grate open area of about $30 \%$. The internal view of the discharge grate is shown in Figure 7-2. Only one grate design was studied in this work.

\subsection{Charge composition}

The particles used in this study to represent mill charge were glass beads. The use of glass beads was considered sufficient, based on the primary goal of the thesis: to develop techniques for estimating porosity in tumbling mills. Another reason for using glass bead was the minimal amount of breakage the glass beads would undergo while the mill was rotating. This follows the assumption that breakage did not occur, for this study.

Glass beads have been used in many other studies representing real systems (for an example, see Santomaso et al. (2003)). It is expected that some refinements in the porosity calculation formulas would have to be done in order to study the packing of real ore. However, the basic principles would remain the same.

\subsubsection{Particle size distribution}

As mentioned earlier (in section 5.4-Estimating the porosity), the charge had a discrete particle size distribution. The particle size distribution was nominally based on typical real 
mill distributions and modified to accommodate the truncation imposed for the purposes of PEPT experiments. In this case, a Rosin-Rammler distribution (see Figure 7-4) was employed. This distribution was based on weight. Since the particle had a uniform density, this is equivalent to using volume as a basis for particle size distribution. This, eventually, is equivalent to using percentage passing, as a measure in determining the distribution.

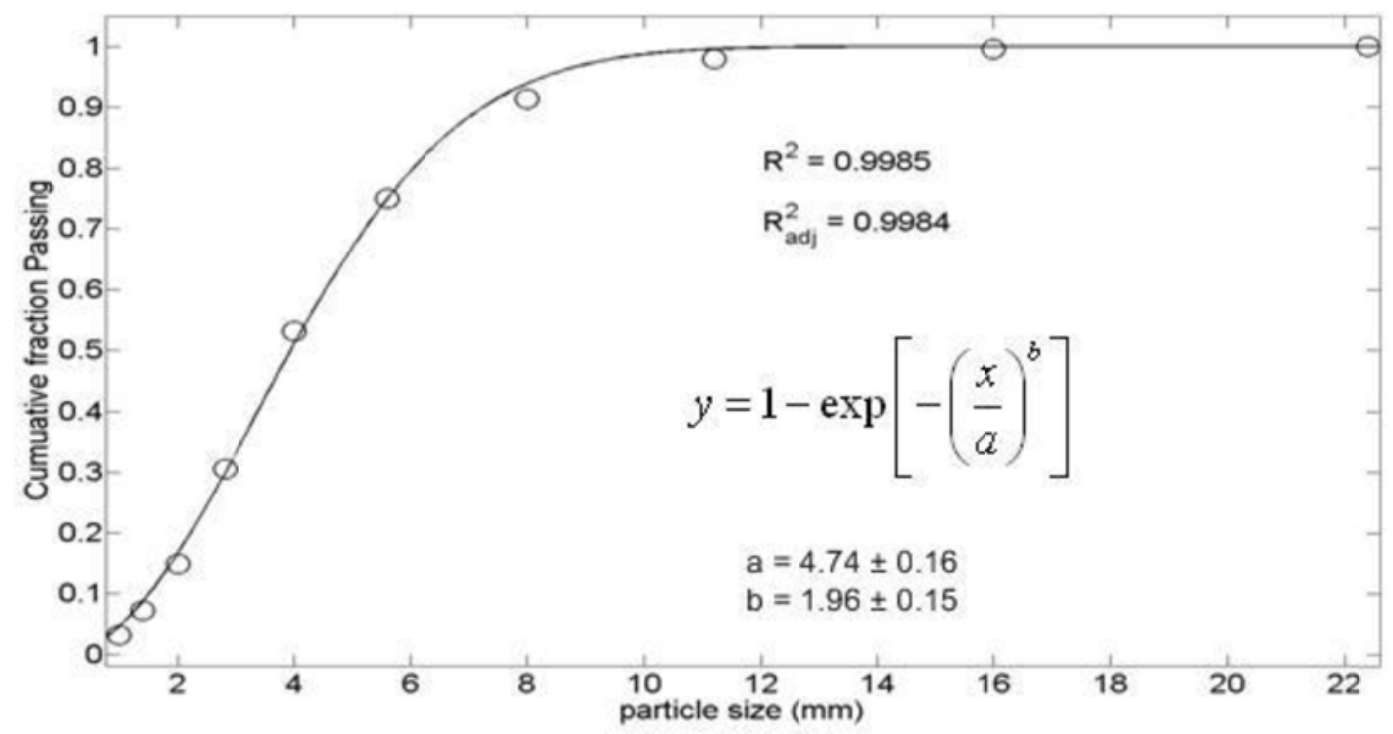

Figure 7-4 Truncated Rosin-Rammler size distribution of glass beads

\subsection{Experimental procedure}

\subsubsection{Positioning the mill between the cameras}

During the experiments, the mill had to be placed between two detectors within the PEPT facility, at the University of Birmingham. Much care was taken in aligning the mill with the detectors. Figure 7-5 shows the experimental mill positioned between the two detectors. 


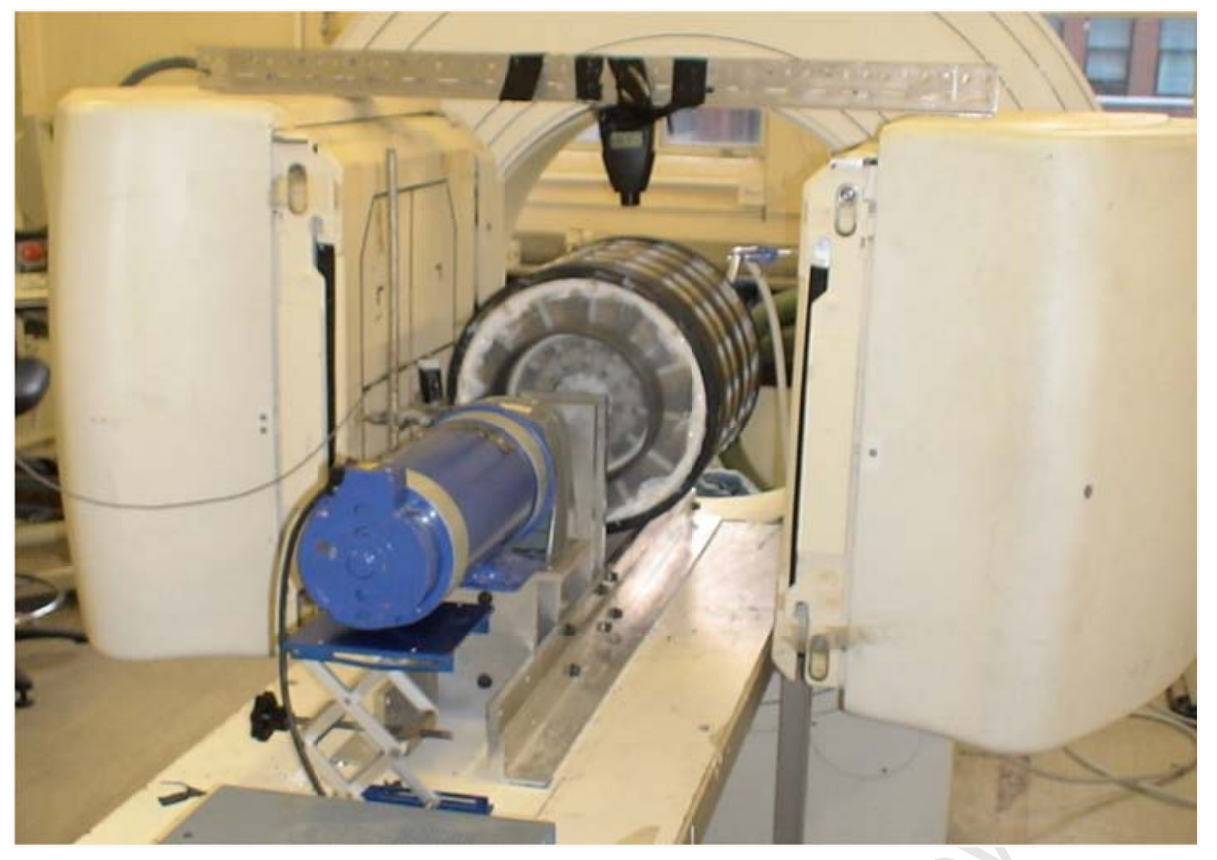

Figure 7-5 The experimental mill placed between detectors

\subsubsection{Labelling the tracers}

The labelling method used in this work is direct activation. This was achieved by bombarding a glass bead with a $33 \mathrm{MeV}{ }^{3} \mathrm{He}$ beam yielding a tracer glass bead containing ${ }^{18} \mathrm{~F}$. This was done at the University of Birmingham Cyclotron. More details on labelling by direct bombardment were discussed in section 4.1.1 - Tracer labelling.

\subsubsection{Mill operational configurations}

Among the aims of this thesis was to study how spatial porosity distribution varies with operational parameters. Thus, the mill was studied under various operating conditions. The operational parameters considered were load fraction and mill speed-as percent of critical speed (\% of crit.).

\subsubsection{Mill speed}

Mill speed is an important parameter in the modelling of tumbling mills. The packing of particles in tumbling mills is highly dependent on mill speed. In order to understand and model the relationship between charge porosity distribution and mill speed, the speed had to be varied between 60 and $90 \%$ of crit. Details are shown in Table 7-1. 


\subsubsection{Load fraction}

Load fraction is a well known factor of slurry transport and mill performance (section 2.1.5.2). To model porosity distribution as a function of load fraction, the load fraction is varied between 12.5 and $50 \%$ filling. The experiment parameter matrix is shown below.

Table 7-1 A summary of parameters studied showing mill speed ( $\%$ of crit.) vs. load fraction ( $\%$ filling).

\begin{tabular}{|c|c|c|c|c|c|}
\hline Filling & $\mathbf{6 0}$ & $\mathbf{7 0}$ & $\mathbf{7 5}$ & $\mathbf{8 0}$ & $\mathbf{9 0}$ \\
\hline $\mathbf{1 2 . 5}$ & $2,3,4,5,6,8$ & $2,3,4,5,6,8$ & $2,3,4,5,6,8$ & $2,3,4,5,6,8$ & $2,3,4,5,6,8$ \\
\hline $\mathbf{2 5 . 0}$ & $2,3,4,5,6,8$ & $2,3,4,5,6,8$ & $2,3,4,5,6,8$ & $2,3,4,5,6,8$ & $2,3,4,5,6,8$ \\
\hline $\mathbf{3 1 . 2 5}$ & $2,3,4,5,6,8$ & $2,3,4,5,6,8$ & $2,3,4,5,6,8$ & $2,3,4,5,6,8$ & $2,3,4,5,6,8$ \\
\hline $\mathbf{3 7 . 5}$ & $2,3,4,5,6,8$ & $2,3,4,5,6,8$ & $2,3,4,5,6,8$ & $2,3,4,5,6,8$ & $2,3,4,5,6,8$ \\
\hline $\mathbf{5 0 . 0}$ & $2,3,4,5,6,8$ & $2,3,4,5,6,8$ & $2,3,4,5,6,8$ & $2,3,4,5,6,8$ & $2,3,4,5,6,8$ \\
\hline
\end{tabular}

From Table 7-1, each data cell shows experiments performed for each mill configuration. For example, the bottom-most right-most cell shows that particles 2, 3, 4, 5, 6 and $8 \mathrm{~mm}$ were tracked. The mill configurations for the cell were mill speed- $-90 \%$ of crit., and filling fraction-50.0\%. Thus, the matrix represents a total of $5 * 5 * 6=150$ experiments and 25 mill configurations.

\subsubsection{Locating the mill shell}

To have meaningful measurements, the mill's boundaries needed to be established. Therefore, there were experiments performed aimed at determining the boundaries of the mill. This was by way of placing a tracer particle on the mill shell (as shown in Figure 7-6). The mill was then run so that several data points at a given axial position were accumulated.

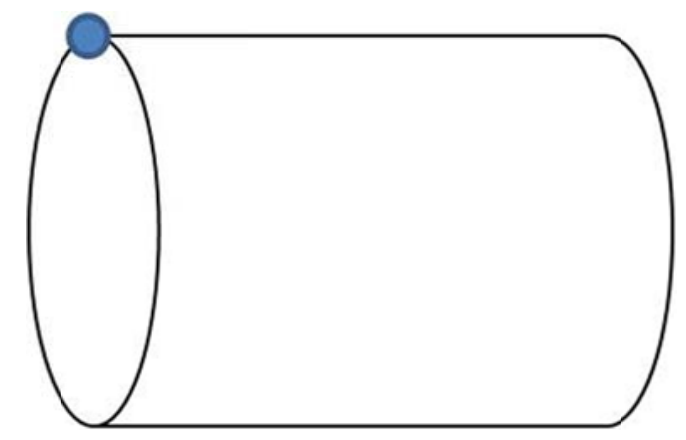

Figure 7-6 Tracer placed on mill shell for locating the mill shell 
This was repeated for two other axial positions, in order to get the outline of the mill shell along the mill length. The centre of the mill was also determined using the mill location data. This was done by fitting ellipses to each of the three datasets. The mill centre coordinates were then used to transform the mill data so that the origin was at the centre of the mill.

\subsubsection{Other conditions: wet or dry milling}

Since the main focus of this work was to develop techniques for calculating the porosity of multi-component particle assemblies, using PEPT data, the mill was operated without slurry. Thus, a dry system was considered sufficient for the purpose of developing the aforesaid techniques. In addition, the studies would still give good indication of the spatial distribution of porosity. Furthermore, all assumptions made in this work are expected to apply to a wet mill, as well. Thus, the studies presented herein provide a good basis for studying wet systems.

\subsubsection{Operating mode: batch or continuous}

The mill was operated in batch mode. This implies that particles were not allowed to flow out of the system. Thus, steady state is ensured by the fact that the particles within the particle bed did not undergo breakage. In addition, the load was also kept constant. Therefore, the data collected in these experiments represents a rotary mill at steady state, sufficiently.

\subsubsection{Length of run}

The experiments were approximately five minutes long. This was considered sufficient because system studied was quite small, compared to the size of particles in it. However, further studies need to be conducted in order to ascertain an optimal amount of time that is required to attain the best possible results.

\subsection{Conclusion}

In this chapter, the experimental programme for this work has been discussed. The experiments discussed above were performed at the University of Birmingham Positron Imaging Centre, using the PEPT technique. The equipment used was a laboratory scale mill which is very similar to real tumbling mills. The charge that was studied was composed of glass beads. To allow for modelling of porosity distribution as a function of operating conditions, the mill was operated as varying mill speeds and load fractions. The result of 
these experiments was refined using techniques in chapter 4 . Thereafter, they were used to compute porosity, using techniques developed in chapters 5 and 6. 


\section{Results}

This chapter presents the results based on computations performed using data from the experimental programme outline in the previous chapter. The computations are performed using formulations presented in chapters 5 and 6 . Furthermore, some implications the results may have on comminution and the study of packing in multi-component mixtures are also presented.

\subsection{Steady state}

The results of the power draw computation use to determine whether the system was at steady state are presented below:

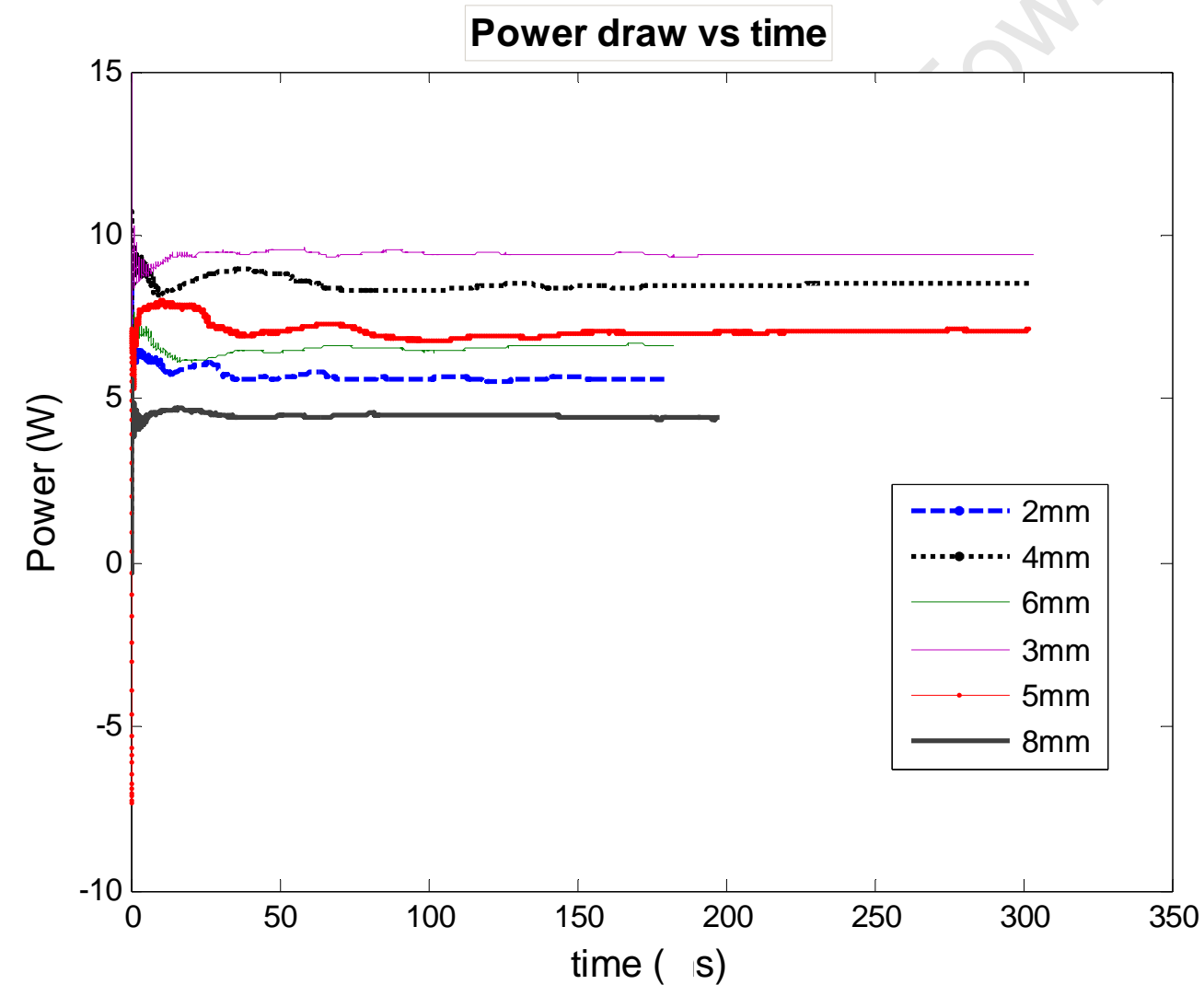

Figure 8-1Variation of Power draw with time

Figure 8-1shows the power draw as it varies with time for individual size components. The result above confirms that each component in the system is at steady state. It also follows that the whole system is at steady state, since the sum of power draw of its individual components is also invariant with time. Therefore, the assumption that the rotary mill is at steady state equilibrium holds. The result also shows that the system does indeed satisfy the coexistence 
property. As can be observed, each component exhibits steady state behaviour despite being a part of a system with multi-components. With this result, the numerical schemes developed earlier can be applied to the study of the mill using PEPT data.

\subsection{The effect of speed on porosity distribution}

In this section, the effect of speed on porosity distribution is discussed. This analysis was done by comparing the spatial distribution of porosity for each load fraction. Firstly, the effect of mill speed on the spatial distribution of porosity for the mill operated with a $31.25 \%$ load is discussed. The results are presented using three methods: spatial distribution plots, probability distributions and radial profiles. These three mechanisms each give valuable information about porosity distribution, in the mill.

\subsubsection{Spatial distribution of porosity at $\mathbf{3 1 . 2 5 \%}$ filling}

The following (Figure 8-2) are plots of spatial distribution of porosity for a $31.25 \%$ load. The results demonstrate how porosity distribution varies with mill speed. In this particular study, five speeds were considered: $60,70,75,80$ and $90 \%$ of crit.

From Figure 8-2, it can be observed that porosity in a rotary mill exhibits a spatial distribution. Furthermore, it was observed that this distribution varies with mill rotation speed. In particular, from Figure 8-2 A through E, the distribution is more evident at higher speeds than it is at lower speeds. In other words, at lower speeds, the mill charge has a more uniform distribution. It was also observed that, at lower speeds, the charge is more compacted and has mostly a porosity of about 0.4 . 

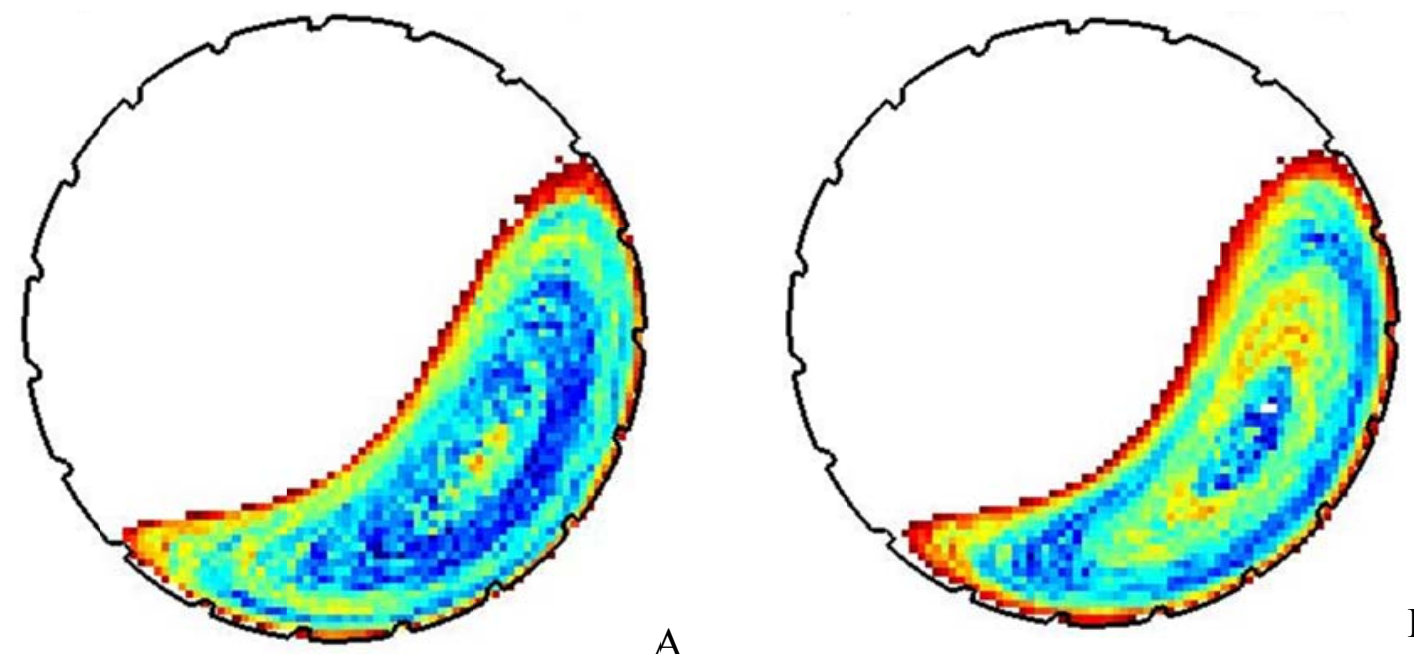

A

$\mathrm{B}$

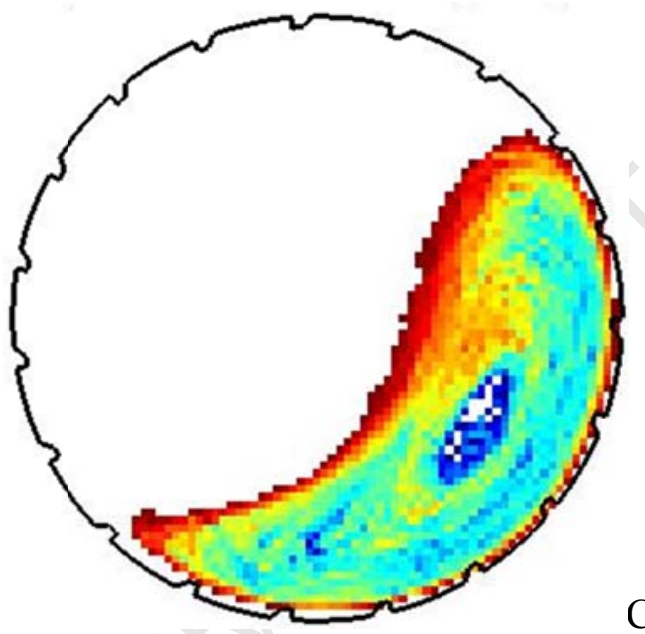

$\mathrm{C}$
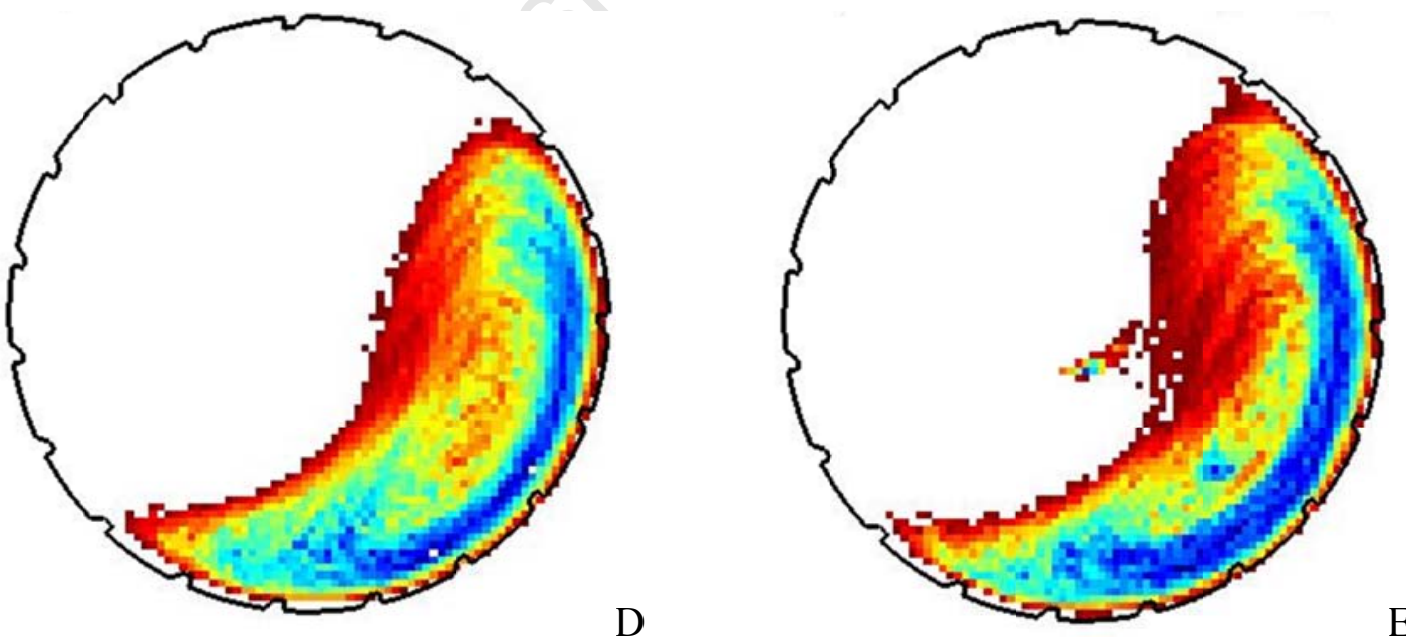

$\mathrm{D}$

E

Figure 8-2 Spatial distribution of porosity at different mill speeds: A) 60 B) 70 C) 75 D) 80 E) 90

From Figure 8-2, it was further observed that at higher speeds, there is a change in the orientation of the spatial distribution of porosity. In particular, in A and B, the region of low porosity is distributed quite evenly across the mill. However, in $\mathrm{C}$, the region seems to not 
exist. On the other hand, in D and E, the charge assumes different profiles from those in A, B and C. In particular, the porosity is generally much higher, though the region of low porosity is more distinct. However, this region lies even closer to the mill shell. A possible explanation for this is that, as mill speed increases, there is a point beyond which centrifugal forces tend to push the rising charge further towards the mill shell. This causes the particles to become more closely packed in that region. However, the charge still continues to cataract, resulting in a more fluid charge away from the mill shell. This results in a higher average porosity.

The phenomenon associated with the band of low porosity can be observed in the study by Arntz et al. (2008). However, it seems to be absent from the work by Yang et al. (2008), as can be seen in Figure 2-14. It is, therefore, postulated that this phenomenon is unique to multi-component systems. The reason for this proposition is that, in multi-components small particles tend to filter through spaces among the large particles as segregation takes place. This form of segregation is expected to occur at higher speeds, where smaller particles tend to pack further away from the core of the charge. (Refer to section 5.3.2-Segregation-for more details). The filling effect of smaller particles, therefore, results in a drop in porosity values in the region near the mill shell.

\subsubsection{Probability functions}

Some of the trends observed above can be illustrated using probability distributions. In this section, probability distributions of porosity are presented for the same conditions as in the previous section. Figure 8-3 demonstrates the distribution of porosity values across the mill at each of the five speeds considered in this work. 


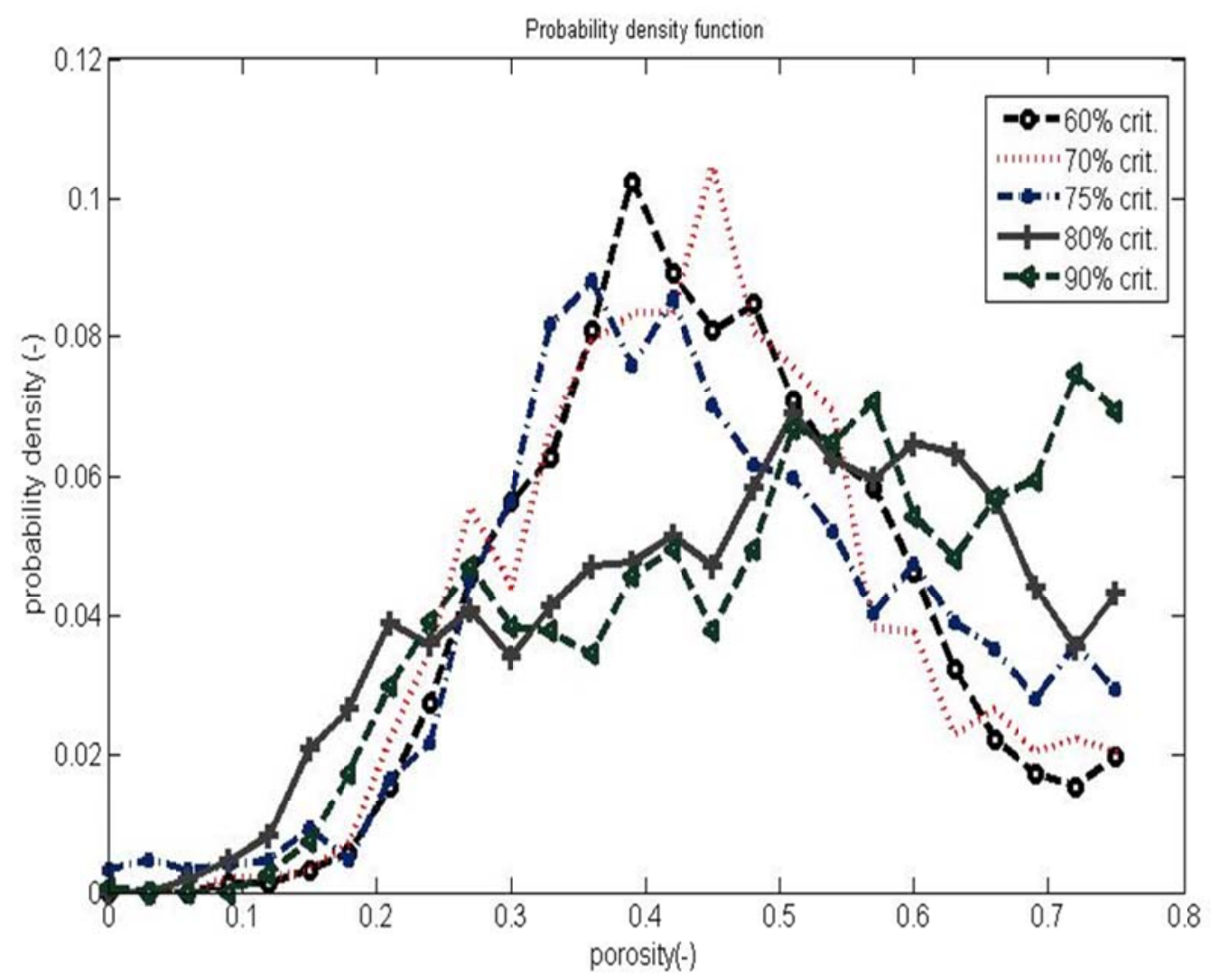

Figure 8-3 Distribution of porosity values at different mill rotation speeds

From Figure 8-3, it can be observed that the probability distribution of porosity values within the mill varies with mill speed. In particular, at higher speeds, the peak of the curve is lower. The lower peak means that there is less of the most probable porosity values for the given mill speed. This implies a wider distribution of porosity values. A wider distribution further implies less uniformity. On the other hand, the range of porosity values in the mill is narrower at lower speeds, where porosity is quite uniform. For instance, the curve with the highest peak is that of the mill operated at $60 \%$ of critical speed. The peak was also observed to be associated with a narrow interval of porosity of about 0.4 . Consequently, the majority of the charge, at that speed, had the same porosity as that of a stationary loosely packed system.

From Figure 8-3, it was also observed that, as speed increases, the width of the distribution also increases in a particular direction: towards higher porosity values. In addition, there is an increase in the probability of higher porosity values. This implies a higher average porosity at higher mill speeds. (Refer to the next section for details). Further, it was observed that the distribution curve at lower speeds is also smoother than it is at higher speeds. This is partly due to an increase in the number of particles in the cataracting zone. Furthermore, the values 
of porosity in the cataracting zone were also included in the computation of porosity. Therefore, at higher speeds, the distribution curve also shows an increase in porosity values close to unity.

The results in Figure 8-3 correspond to those in Figure 8-2, where it was observed that the spatial distribution pattern varies with mill speed. In particular, it moves from being uniform to having a well defined distribution. The next section discusses the correlation between average porosity and mill rotation speed.

\subsection{Average porosity vs Mill rotation speed}

The figure below is a plot of average porosity against mill rotation speed. This plot was made using values corresponding to the results presented in the two previous sections.

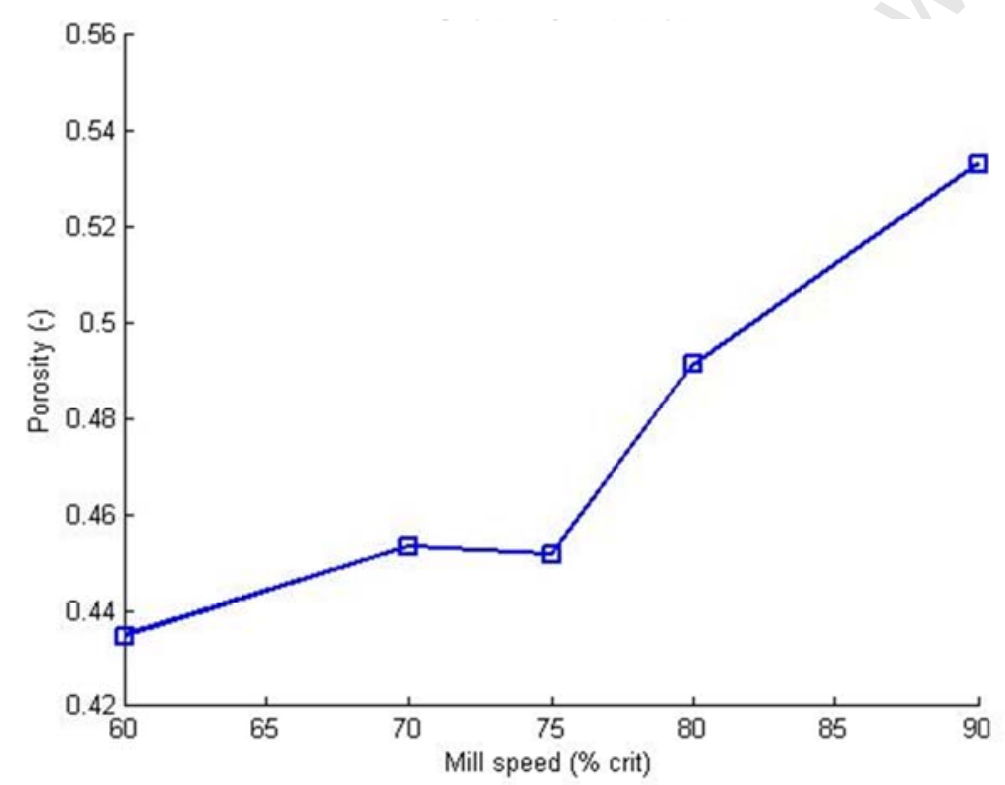

Figure 8-4 Average porosity vs mill speed

From Figure 8-4, it can be observed that there is a correlation between average porosity and mill speed. In particular, the average porosity increases with mill rotation speed. This result confirms the assertions made in the previous sections.

\subsubsection{Radial profiles}

Radial profiles give additional insight into the results presented in the previous sections. The analysis in this section is based on radial profiles of porosity shown in Figure 8-6 below. Radial profiles give an indication of the variation of porosity with radial position within a 
given reference region. Thus, to compute these profiles, a small region of the mill—regarded as relevant to this analysis - was studied. This region is shown in Figure 8-5 below.

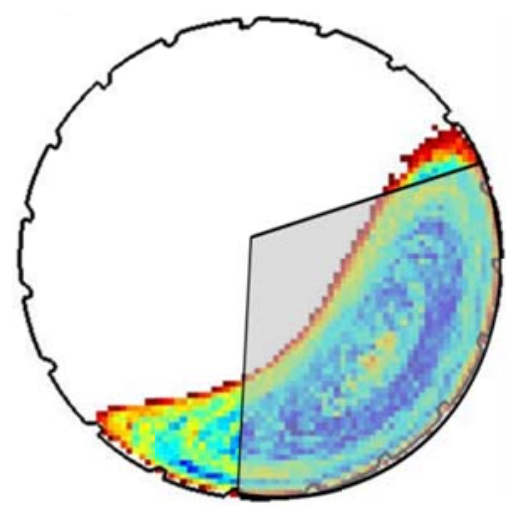

Figure 8-5 Relevant region when computing radial profiles

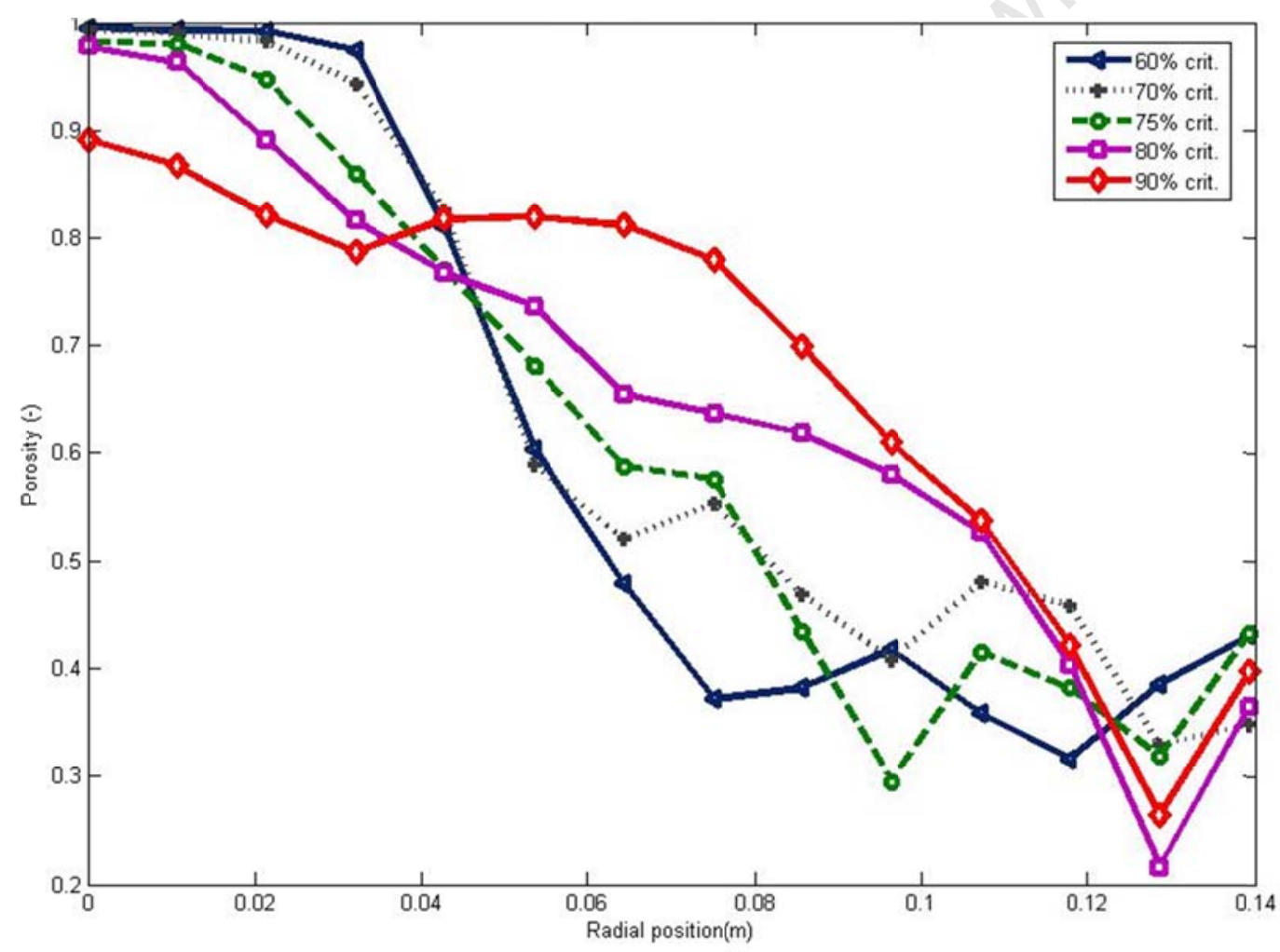

Figure 8-6 Radial profile of porosity at different speeds

From Figure 8-6, it was observed that there is a variation in porosity with radial position. Generally, porosity decreases with an increase in radial distance, from the charge free surface going towards the mill shell. In Figure 8-6, for example, the left most part of the curvewhich is near the centre of the mill- has porosity values close to unity. This represents the region outside of the charge or rather above the free surface. The free surface is represented by the region near radial position $0.04 \mathrm{~m}$, which represents a transition region. This region 
generally reflects a change in porosity from values greater than 0.8 to values less than 0.7 . This, however, varies with mill rotation speed.

The region near the free surface is more fluid, since particles in that region flow more freely than those near the mill shell. This implies that the region near the free surface is more loosely packed. The region near the free surface, therefore, has high porosity.

The trend of decreasing porosity towards the mill shell is broken near the shell. The drop in porosity towards the shell is mostly due to centrifugal forces in the rising charge. On the other hand, the sudden increase in porosity in the region very near to the mill shell is due to the lifters that were not accounted for when computing the porosity.

Furthermore, Figure 8-6 shows that the radial profile of porosity varies with mill rotation speed. In particular, at lower speeds, there is a wide radial region of low porosity, with values averaging 0.4. The free surface is also narrower at lower speeds than it is at higher speeds. However, at higher speeds, the region of low porosity becomes narrower. Accordingly, the free surface and the region of higher porosity become much wider. For mill speeds of about 80 and $90 \%$ of crit. the narrow band of low porosity becomes more prominent. This is shown by the narrow region in the radial profile towards the mill shell. In this region, there is a sudden drop in porosity and the minimum value for the distribution curve occurs in this region.

In addition, it was also observed that, the rate of change of porosity, as radial distance increases, depends on mill speed. At lower speeds the gradient is less steep compared to higher speeds in the core of the charge and steeper near the free surface. On the contrary, at higher speeds, the gradient is steepest within the core and less steep near the free surface. This implies that, at high speeds, the charge is mostly fluid. In addition, there is a sudden drop in porosity near the shell—or in the narrow band of low porosity, in particular.

The results presented above provide valuable insight into the dynamics of rotary mill operations and open avenues for comminution optimisation. In particular, it was observed that, at higher speeds the region of low porosity near the mill shell would limit the slurry transport in that region. At $75 \%$, this region is not very evident and porosity is much higheron average - compared to lower speeds. Therefore, in terms of charge porosity and its spatial distribution, the mill speed that would give optimal slurry flow is about $75 \%$ of crit. 


\subsection{The effect of load fraction on spatial distributions of porosity}

To study the relationship between load fraction and porosity distribution, spatial plots for four different load fractions were analysed. The figure below shows the spatial distribution plots for the following load fractions: 12.5, 25.0, 31.25, and 50.0\%. These experiments were all performed at the same mill rotation speed: $75 \%$ of crit.

A

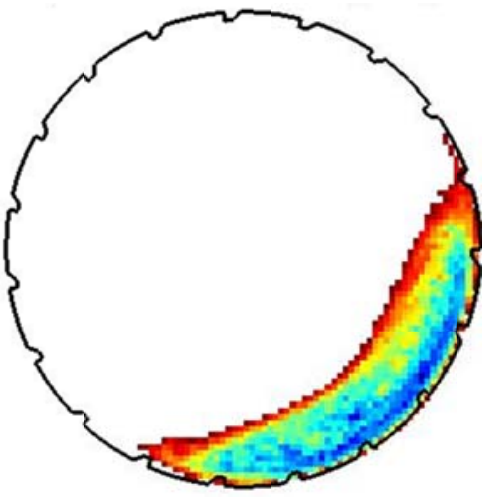

$\mathrm{C}$

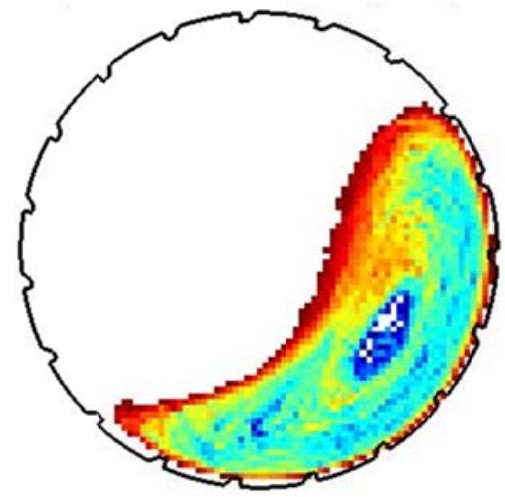

B

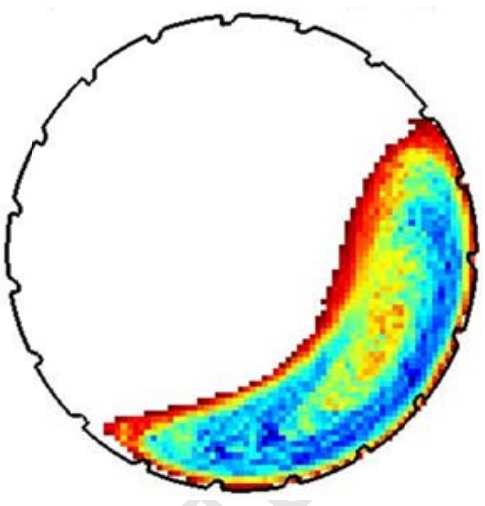

$\mathrm{D}$

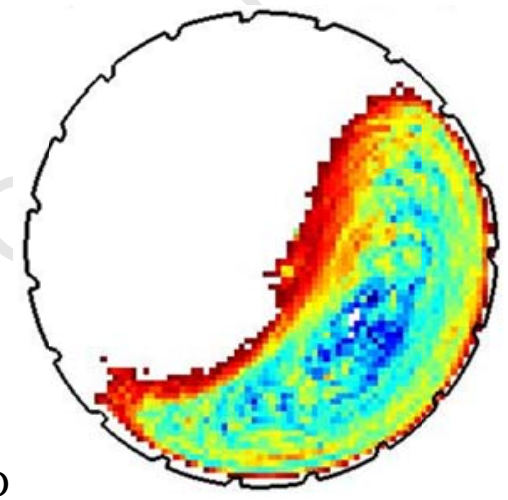

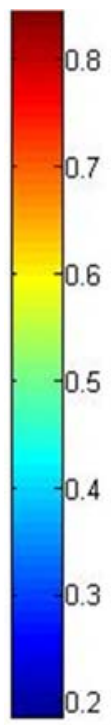

Figure 8-7 Variation of spatial distribution of porosity with load fraction

From Figure 8-7, it is evident that the spatial distribution of porosity varies with mill load fraction - if mill speed is fixed. Firstly, the profile of the distribution is different in the four plots shown above. The high load fractions-31.25\% and 50\% load-seem to have a more uniform distribution compared to lower load fractions. For low load fractions- $12.5 \%$ and $25 \%$ load - the charge has regions of well defined porosity distribution. This also implies a greater extent of segregation. On the other hand, the higher load fractions seem to exhibit more mixing. Thus, the charge, at $75 \%$ of crit., has a more mixed distribution for high load fractions than that exhibited by lower load fractions.

Secondly, for low load fractions, the narrow band of low porosity near the mill shell would have already formed at $75 \%$ of crit. There is, however, no evidence of this band for higher load fractions for the same speed. Thus, low load fractions require a lower speed to form the 
narrow band of low porosity, than higher load fractions. This is due to the fact that, the charge with a higher mass is more resistant to motion or the action of centrifugal forces.

Thirdly, the shape of the profile of the free surface varies with load fraction. This has much to do with the volume of charge. Generally, as mentioned above, the charge with a higher load fraction offers greater resistance to change in shape of the free surface than lower load fraction. This is shown by the higher curvature and more defined S-shape of the free surface profile for higher load fraction. Conversely, for lower load fractions, the charge centrifuges more easily than it does for higher load fractions. In Figure 8-7 (A), for instance, the shape of the free surface is very similar to that of the mill shell.

From the foregoing, the effect of mill speed on porosity distribution depends on the load fraction. Thus, there is great value in studying the combined effect of mill rotation speed and load fraction on porosity distribution. This is presented in the next section.

\subsection{The combined effect of mill speed and load fraction on porosity}

An important study of porosity in rotary mills is to establish the combined effect of mill rotation speed and load fraction. From the literature (see section 2.1.5.2), it has been established that the effect of mill speed on slurry transport is dependent on mill filling fraction. Consequently, there is a relationship between mill speed, filling fraction and porosity. It is, therefore, important to get insight into the variation of porosity with both filling fraction and mill rotation speed. This would improve our understanding of slurry transport as it varies with both mill speed and load fraction. In the two previous sections 8.1 and 8.4 , the filling fraction and mill speeds, respectively, were kept constant. Thus, only the effect of one variable on porosity could be studied. In this section, the effect of one variable on correlation between the other variable and porosity is discussed. Most importantly, the effect of filling fraction on the variation of porosity distribution with mill speed was studied.

The figure below shows plots of spatial distribution of porosity as it varies with mill speed at three different filling fractions. Each row represents spatial plots for the same speed but for three filling fractions. Conversely, each column represents plots for the same filling fraction but for five speeds. 

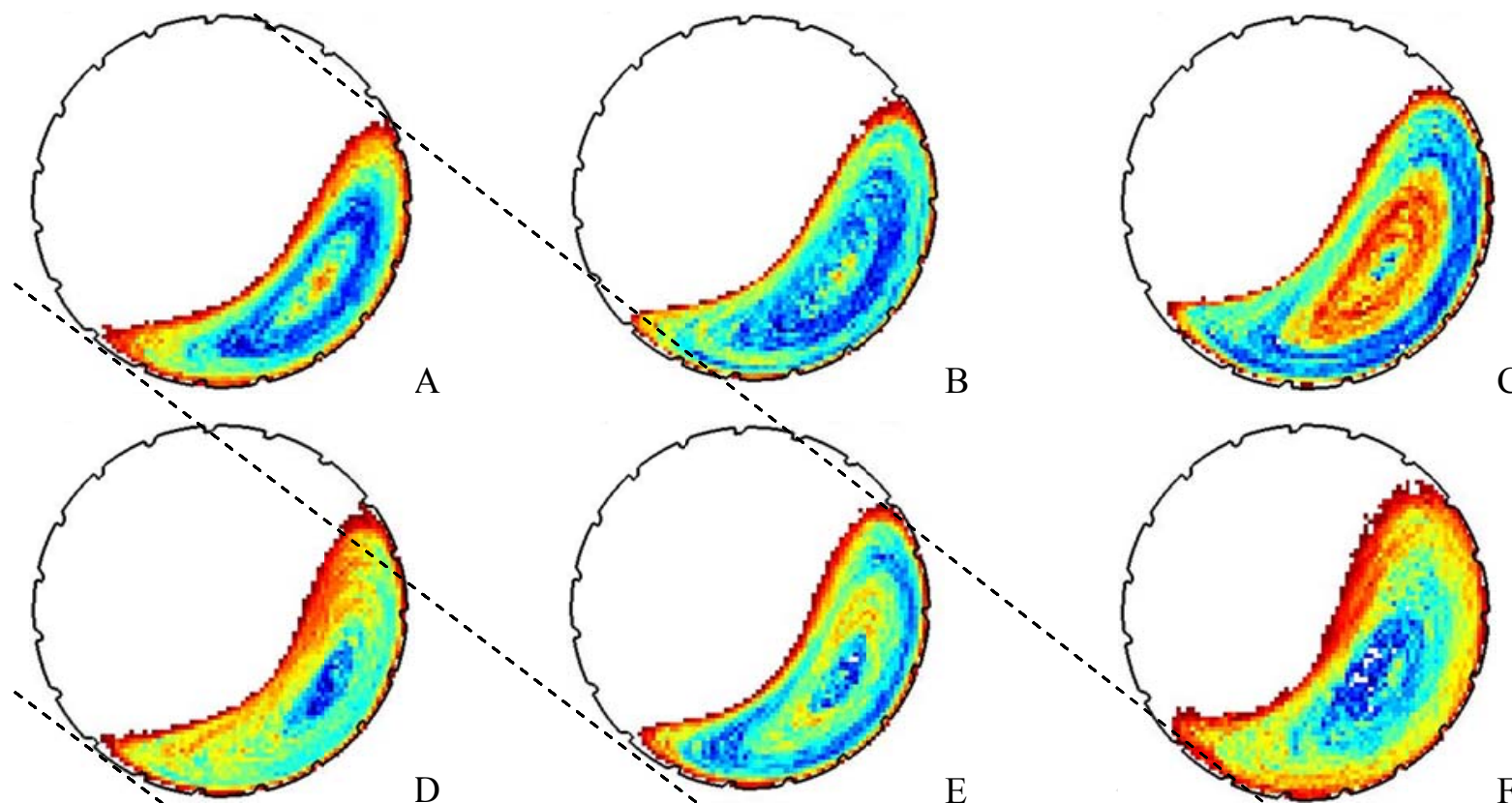

B

$\mathrm{C}$
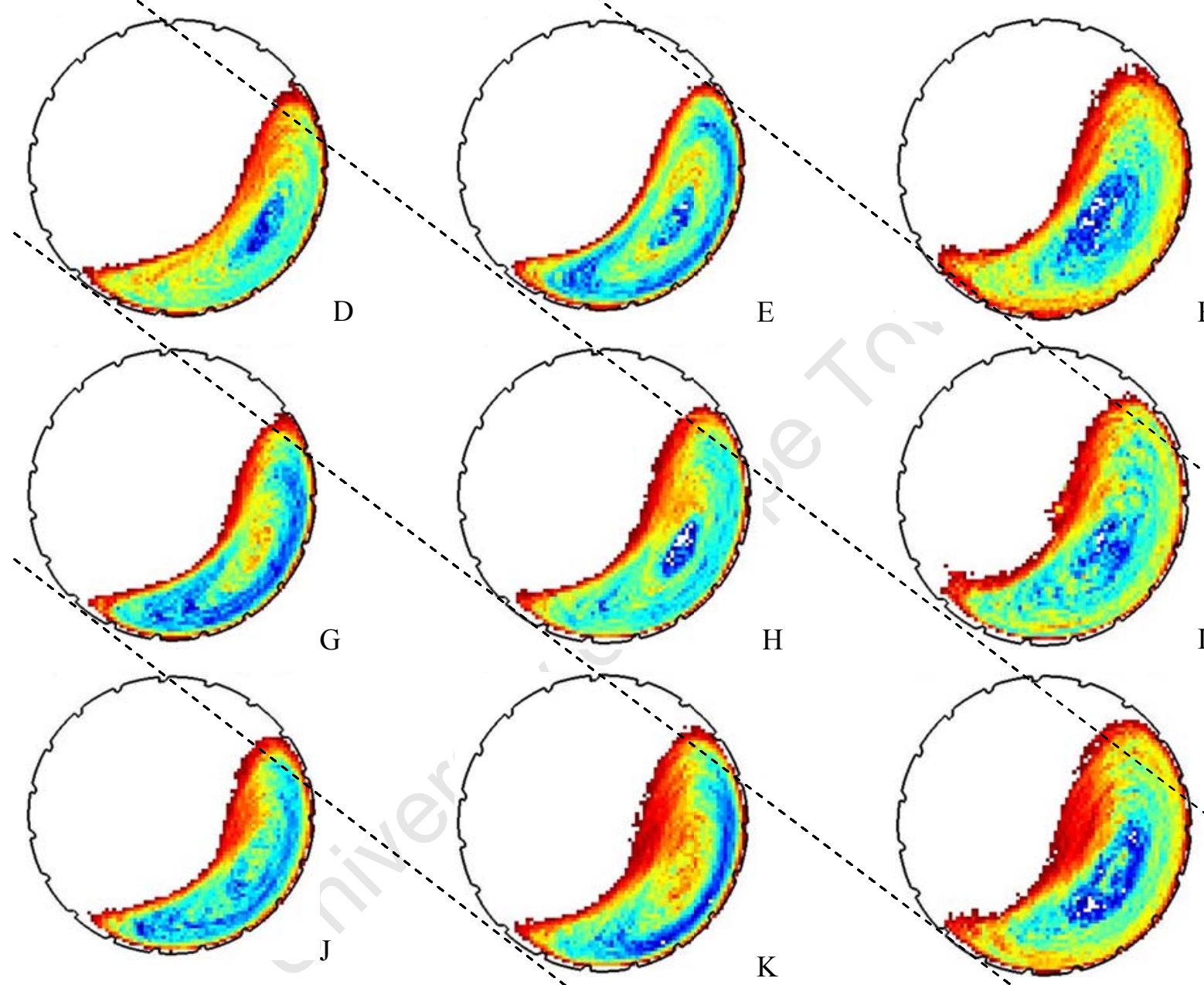

F
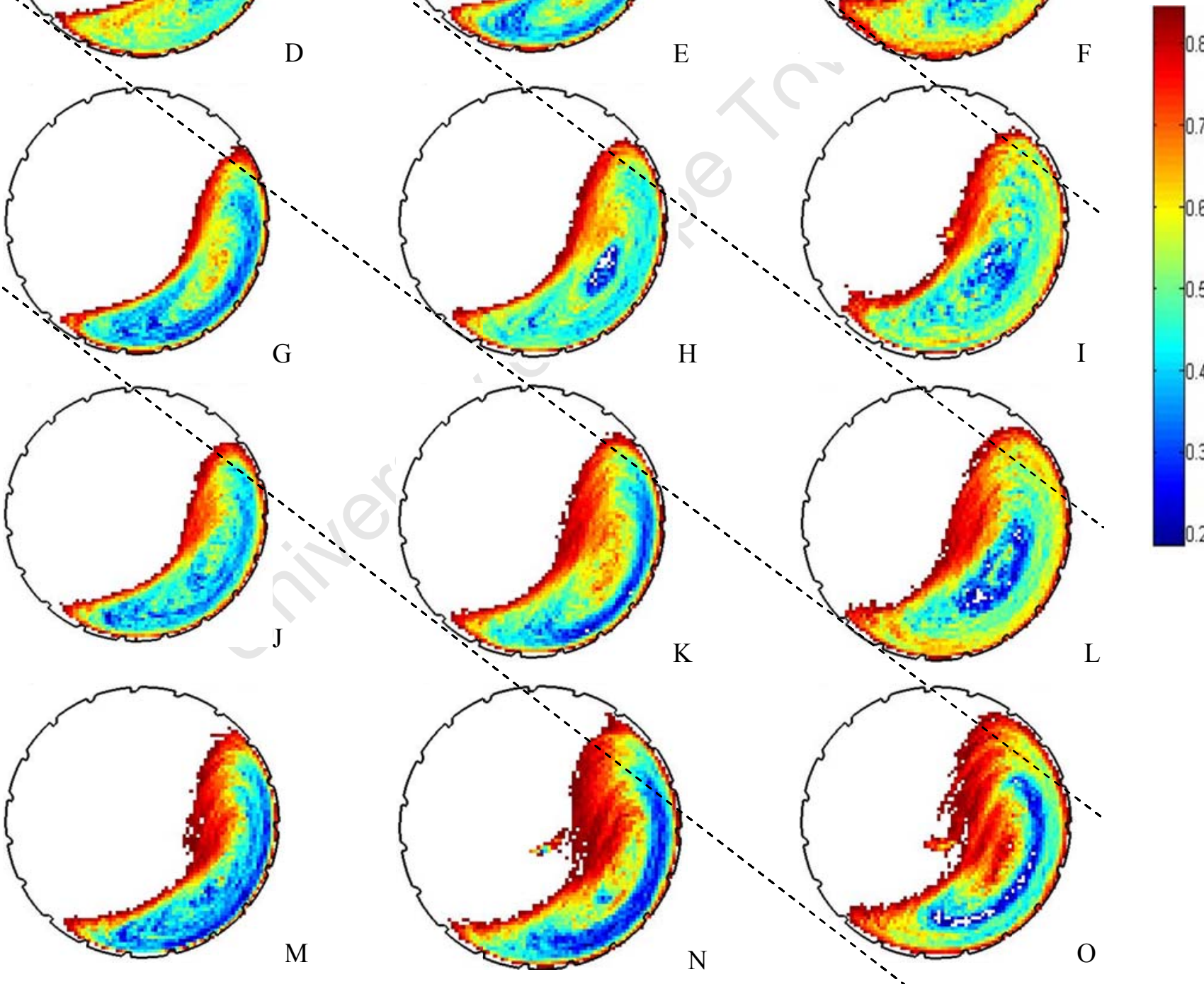

$\mathrm{L}$

Figure 8-8 Spatial distribution plots showing the combined effect of mill speed and load fraction on porosity 
From Figure 8-8, it can be observed that porosity distribution varies along rows. This implies that, for the same speed, porosity distribution varies with filling fraction. This is also true for (along) columns. For the same filling fraction, the effect of mill speed on porosity is apparent. These variations have already been discussed in sections 8.4 and 8.1, respectively.

Further analysis of the plots across both axes-diagonally-reveals some patterns corresponding to a combined effect of filling fraction and mill speed on porosity. For example, when G, H and I are compared, 'I' seems to maintain a more uniform distribution compared with $\mathrm{H}$. $\mathrm{H}$, accordingly, also has a more uniform distribution than $\mathrm{G}$. Thus, for a lower filling fraction, a lower speed is required to move from a uniform distribution to a more varied distribution. Equally, as the filling fraction increases, a higher speed is required to produce porosity distribution similar to that produced at lower speeds for lower filling fractions.

Following the last point above, the effect of mill filling fraction on the variation of porosity distribution with mill speed, and vice versa, can be established further. The former can be observed in the formation of the band of low porosity that occurs near the shell. In particular, at $25 \%$ filling, the band forms at $75 \%$ of crit. (in G), while at $31.25 \%$ filling, the band forms at a higher speed-about $80 \%$ of crit. (in K). Similarly, at $37.5 \%$ filling, the band forms at a much higher speed—at $90 \%$ of crit. (in O).

Thus, for a low filling fraction, a lower speed is required for the lower band of porosity to form towards the mills shell.

From the foregoing, it should be noted that there are similarities in spatial distribution pattern across diagonal elements, rather than along rows or columns. For instance, the spatial distributions in $\mathrm{D}, \mathrm{H}$ and $\mathrm{L}$ are similar. Accordingly, the plots a step above each of these plots, namely A, E and I, are also similar. Below D, H and L are G, K and O, which also follow a similar pattern. This confirms the pattern observed in $\mathrm{D}, \mathrm{H}$ and $\mathrm{L}$. Therefore, it can be concluded that the effect of mill rotational speed on porosity distribution depends on filling fraction.

From the above analysis, it can be shown visually that conditions near those of $\mathrm{D}, \mathrm{H}$ and $\mathrm{L}$, respectively, are transition points between quite well mixed and extensively segregated charge. The explanation for these observations was given in section 8.4. 


\subsection{Conclusion}

In this chapter, it has been established that the porosity of multi-component mixtures can be derived from single particle trajectory data. Further, it has been demonstrated that when a mill is operated at even relatively low speeds, there is evidence of a spatial distribution. This shows that assumptions made when developing slurry transport models that the porosity is uniform at 0.4 do not hold. Thus, it is important to study how porosity varies across the mill and with operating parameters. From the results presented above, the porosity distribution varies with both mill speed and load fraction, even when one of them is fixed.

In particular, spatial distribution changes with changes in mills speed. The shape of the charge also varies with both mill speed and load fraction. As speed increases, there is an associated increase in the probability of higher porosity values. This results in an increase in average porosity. The results also showed that the variation of porosity with mill speed depends on the load fraction. For higher load, the variation is not as much as it is for lower load fraction. Furthermore, for low load fractions, a lower speed is required to produce the trends similar to those observed in higher load fractions at higher speeds.

The results presented in this section are consistent with theory. This shows that the assumptions made and the mathematical formulations developed in chapters 5 and 6 are valid. 


\section{Conclusions}

This chapter is a conclusion of the thesis, which also offers recommendations for future work.

\subsection{Thesis outcome}

This thesis was conducted to address the following hypotheses:

- The time-averaged packing density provided by a tracer equals the equilibrium average packing density of its size component, because packing density is invariant at steady state and a particle tracer represents other particles from its size component.

- The porosity of any given volume element within the charge at steady state is a linear combination of solid volume contributions of individual size components, due to coexistence of the individual components within each volume element.

- Porosity decreases with increasing radius from the free surface of the charge, due to an associated increase in particle number density towards the mill shell.

- Porosity increases with an increase in mill speed due to charge dilation, which results in a reduction in packing density, on average.

The objectives of this thesis were as follows:

- Develop mathematical and numerical frameworks for calculating (from PEPT trajectory fields):

- Solid volume contribution of each size component in a given volume element;

- Porosity distributions through the combination of the various size components based on the solid volume distributions computed above;

- Study the variation of porosity distribution with respect to:

- Radial position from the free surface across a relevant region of the mill;

- Mill speed;

- Filling fraction. 
Based on the objectives above, the following are the outcomes of the thesis:

- Mathematical formulations for computing porosity of multi-components in rotary mills, using single-particle trajectory data, have been developed. These were based on three basic assumptions: ergodicity, steady-state equilibrium and coexistence of multicomponents. (See chapter 5 for details).

- A theoretical analysis of these assumptions was also done-in chapter 5.

- Numerical frameworks based on the mathematical formulations above were also developed using MATLAB. (See chapter 6 for details).

- PEPT experiments were performed to collect data for porosity computations. In all, 150 PEPT experiments were performed. (See chapter 7 for details).

- Porosity was computed using the numerical framework mentioned above.

- The effects of mill speed and filling fraction, respectively, on porosity distribution were studied. The combined effect of the two parameters above was also studied qualitatively. (See chapter 8 for details).

The following conclusions have been reached:

- The porosity of charge in a mill is not uniform: it exhibits a spatial distribution. This was shown by spatial plots, probability density plots and radial profiles of porosity. Therefore, to understand slurry transport, there is need to understand how porosity is distributed across the mill.

- Single particle trajectory data can and has been used in computing the porosity of charge in a mill. This can be observed from the results presented in chapter 8 , which are consistent with theory.

- The porosity distribution in a rotary mill becomes less uniform as speed increases.

- The effect of mill speed on porosity distribution depends on mill filling fraction. In other words, there is a combined effect of mill speed and filling fraction on porosity distribution. This interaction of the two variables results in better understanding of the best conditions at which to operate the mill.

- At higher speeds, there is a band of lower porosity that forms within the charge near the mill shell, due to increase in centrifugal forces. This feature is more evident in multi-component systems than it is in monosized systems. This band can greatly affect slurry flow at higher speeds as it forms in a region where slurry transport is expected to occur. 
- The results have great value in understanding slurry transport in rotary mills. From this study, the optimal slurry transport would exist at around $75 \%$ of crit., for a $31.25 \%$ load. As the mill rotation speed increases to $80 \%$ and $90 \%$ of crit. the porosity in the region of flow near the shell decreases. Therefore, slurry transport in this region also decreases. However, the optimal speed for operating mills depends on filling fraction.

The results present endless opportunities for providing insight into the dynamics of multicomponent mixtures in rotary mills and similar systems. Better understanding of the distribution of porosity is also enhanced due to formulations that are based on measured particle level data in a real system.

In addition, with such computations that are based on sound experimental procedures, it is also possible to verify assumptions made in the design of numerical models, such as DEM and similar technologies.

\subsection{Limitations}

- The system that could be studied was of a limited size; in particular, a laboratory scale mill. The expectation is that the assumptions made in this work would still hold for bigger system. The formulations would also be applicable to bigger systems.

- However, the formulations did not take care of the axial variations in porosity that may occur in the mill.

- In addition, the lifters were also not considered explicitly in the formulations.

- The formulations were also limited to mixtures having a discrete size distribution.

- Another limitation is the straight line fitting method used to allocate residence time between consecutive locations. This method may not always be accurate. There is, therefore, lots of room for improvement by way of fitting splines.

\subsection{Future work}

- This work was focused on studying the transverse section of the mill. Thus, the spatial distributions were also based on an assumption that porosity does not vary much along the axial axis. It is proposed that the axial distribution of porosity be studied to verify this assumption. 
- The methods developed in this work where tailored for systems having a discrete particle size distribution. It would be nice to extend these formulations to include continuous particle size distributions.

- The sensitivity of the formulations used in this work still has to be established.

- Studying a mill fed with real ore would provide insight into the dynamics of real ore systems. It would also be useful to note how results differ from the results presented herein.

- If the limitation of mill size that can be studies using PEPT can be overcome, it would be very insightful to study bigger mills. It would be interesting to see how results for bigger mills would come out and to see if the assumptions made in this study would still be valid for such systems.

- Formulations that explicitly consider lifters also need to be developed.

- There is need to establish the optimal amount of time required to more accurate results.

- Finally, in order to be integrated within a transport modelling equation, a quantitative description - a model — is needed for the variation of spatial distribution of porosity with and key operating parameters. 


\section{REFERENCES}

Agrawala, S, R K Rajmani, P Songfack, and B K Mishra. "Mechanics of media motion in tumbling mills with 3D discrete element method." Minerals Engineering 10, no. 12 (1997): 215-227.

Arnold, V I, and A Avez. Ergodic Problems of Classical Mechanics. Addison-Wesley, 1989.

Arntz, M M, W K den Otter, W J Briels, P J Bussmann, H H Beeftink, and R M Boom. "Granular Mixing and Segregation in a Horizontal Rotating Drum: A Simulation Study on the Impact of Rotational Speed and Fill Level." American Institute of Chemical Engineers 54, no. 12 (2008): 3133-3146.

Bakalis, S, P W Cox, A B Russell, D J Parker, and P J Fryer. "Development and use of positron emitting particle tracking (PEPT) for velocity measurements in viscous fluids in pilot scale equipment." Chemical Engineering Science 61 (2006): 1864-1877.

Baumann, G, I M Janosi, and D E Wolf. "Particle Trajectories and Segregation in a TwoDimensional Rotating Drum." Europhysics Letters 27, no. 3 (1994): 203-208.

Bird, R B, W E Stewart, and E N Lightfoot. Transport Phenomena. New York: John Willey and Sons, 1960.

Bloch, F, and J D Walecka. Fundamentals of statistical mechanics. London: World Scientific Publishing Co., 2000.

Brin, M, and G Stuck. Introduction to dynamical systems. Cambridge, 2002.

Cleary, Paul W, Matt Sinnott, and Rob Morrison. "Prediction of slurry transport in SAG mills using SPH fluid flow in a dynamic DEM based porous media." Minerals Engineering, no. 19 (2006): 1517-1527.

Cox, P W, S Bakalis, H Ismail, R Forster, D J Parker, and P J Fryer. "Visualisation of threedimensional flows in rotating cans using positron emission particle tracking (PEPT)." Journal of Food Engineering 60 (2003): 229-240.

Cundall, P A, and O D L Strack. "A discrete numerical model for granular assemblies." Geotechnique 29 (1979): 47-65. 
Darcy, H. Les Fontaines Publiques de la Ville de Dijon. Paris: Dalmount, 1856.

de Oliveira, C R, and Thiago Werlang. "Ergodic hypothesis in classical statistical mechanics." Revista Brasileira de Ensino de Fisica 29, no. 2 (2007): 189-201.

Depypere, F, J G Pieters, and K Dewettinck. "PEPT visualisation of particle motion in a tapered fluidised bed coater." Journal of Food Engineering 93 (2009): 324-336.

Dias, R P, J A Teixeira, M G Mota, and A I Yelshin. "Particulate Binary Mixtures: Dependence of Packing Porosity on Particle Size Ratio." Ind. Eng. Chem. Res. 43 (2004): 7912-7919.

Ding, Y L, J P Seville, R Forster, and D J Parker. "Solids motion in rolling mode rotating drums operated at low to medium rotational speeds." Chemical Engineering Science, no. 56 (2001): 1769-1780.

Ding, Y L, R Forster, J P Seville, and David J Parker. "Segregation of granular flow in the transverse plane of a rolling mode rotating drum." Internation Journal of Multiphase Flow, no. 28 (2002): 635-663.

Djordjevic, N, F N Shi, and R Morrison. "Determination of lifter design, speed and filling effects in AG mills by 3D DEM." Minerals Engineering 17 (2004): 1135-1142.

Duan, J, and B Goldys. "Ergodicity of stochastically forced large scale geophysical flows." IJMMS (Hindawi Publishing Corp) 28, no. 6 (2001): 313-320.

Dury, C M, and G H Ristow. "Competition of mixing and segregation in rotating cylinders." Physics of Fluids 11, no. 6 (1999): 1388-1394.

Ergun, S. "Fluid Flow Through Packed Columns." Chem. Eng. Progr. 48 (1952): 89-94.

Evans, R D, and F Civan. "Characterisation of Non-Darcy Multiphase Flow in Petroleum Bearing." Technical Report, University of Oklahoma, 1994.

Fan, X, D J Parker, and M D Smith. "Enhancing 18F uptake in a single particle for positron emission particle tracking through modification of solid surface chemistry." Nuclear Instruments and Methods in Physics Research A 558 (2006b): 542-546. 
Fan, X, D J Parker, and M D Smith. "Labelling a single particle for positron emission particle tracking using direct activation and ion-exchange techniques." Nuclear Instruments and Methods in Physics Research A 562 (2006a): 345-350.

Forchheimer, Ph. "Wasserbewegung durch Boden, Zeitz. ver." Deutsch Ing. 45 (1901): 1731.

German, R M. Particle Packing Characteristics. New Jersey: Metal Power Industries Federation, 1989.

Govender, I, and M S Powell. "An empirical power model derived from 3D particle tracking experiments." Minerals Engineering, no. 19 (2006): 1005-1012.

Govender, I, M S Powell, and G N Nurick. "3D particle tracking: A Rigorous Technique for Verifying DEM." Minerals Engineering 14, no. 10 (2001): 1329-1340.

Hawkesworth, M R, D J Parker, P Fowles, J F Crilly, N L Jefferies, and G Jonkers. "Nonmedical applications of a positron camera." Nuclear Instruments and Methods in Physics Research A 310 (1991): 423-434.

Hill, K M, and J Kakalios. "Reversible axial segregation of binary mixture of granular materials." Physical Review E 49, no. 5 (1994): R3610-R3614.

Hogg, R, and Z Rogovin. "Mass Transport in Wet Overflow Ball Mills." XIV International Mineral Processing Congress. Toronto, 1982.

Jain, N, J M Ottino, and R M Lueptow. "Regimes of segregation and mixing in combined size and density granular systems: an experimental study." Granular Matter 7 (2005): 69-81.

Klimpel, R C, L G Austin, and R Hogg. "The mass transport of slurry and solid in a laboratory overflow ball mill." Minerals and Metallurgical Processing, 1989: 73-78.

Kuo, H P, P Y Shih, and R C Hsu. "Coupled Axial-Radial Segregation in Rotating Drums with High Fill Levels." American Institute of Chemical Engineers AIChE J, no. 52 (2006): $2422-2427$.

Latchireddi, S. Modelling the performance of grates and pulp lifters in autogenous and semiautogenous mills. PhD Thesis, University of Queensland, Australia, 2002.

Latchireddi, S, and S Morrell. "Slurry flow in mills with TCPL — An efficient pulp lifter for AG/SAG mills." International Journal of Mineral Processing, no. 79 (2006): 174-187. 
Latchireddi, S, and S Morrell. "Slurry flow in mills: Grate-only discharge mechanism (part1)." Minerals Engineering, no. 16 (2003): 625-633.

Lawal, A A. "Applications of Sensitivity Analysis in Petroleum Engineering." MSc Thesis, The University of Texaz at Austin, 2007.

Lenci, Marca. "Aperiodic Lorentz gas: recurrence and ergodicity." Ergodic Theory and Dynamical Systems (Cambridge University Press), no. 23 (2003): 869-883.

Liu, S, and Z Ha. "Prediction of random packing limit for multimodal particle mixtures." Powder Technology 126 (2002): 283-296.

McNeil, P A, T D Fryer, M R Hawkesworth, and D J Parker. "Modelling the factors affecting image quality for the RAL-Birmingham positron camera." Nuclear Instruments and Methods in Physics Research A 348 (1994): 593.

Mishra, B K. "A review of computer simulation of tumbling mills by the discrete element method: Part II- Practical applications." International Journal of Mineral Processing 71 (2003): 95-112.

Morrell, S, and I Stephenson. "Slurry discharge capacity of autogenous and semi-autogenous mills and the effect of grate design." International Journal of Mineral Processing, no. 46 (1996): 53-72.

Moys, M. "The effect of grate design on the behaviour of grate-discharge grinding mills." International Journal of Mineral Processing, no. 18 (1986): 85-105.

Napier-Munn, T J, S Morrell, R D Morrison, and T Kojovic. Mineral Comminution Circuits. Their operation and optimisation. Julius Kruttschnitt Mineral Research Centre (JKMRC), 1996.

Ouchiyama, N, and T Tanaka. "Porosity Estimation from Particle Size Distribution." Ind. Eng. Chem. Fundam. 25 (1986): 125-129.

Parker, D J, A E Dijkstra, T W Martin, and J P Seville. "Positron emission particle tracking studies of spherical particle motion in rotating drums." Chemical Engineering Science 52, no. 13 (1997a): 2011-2022. 
Parker, D J, and X Fan. "Positron emission particle tracking-Application and labelling techniques." Particuology 6 (2008): 16-23.

Parker, D J, C J Broadbent, P Fowles, M R Hawkesworth, and P McNeil. "Positron emission particle tracking - a technique for studying flow within engineering equipment." Nuclear Instruments and Methods in Physics Research A 326 (1993): 592-607.

Parker, D J, et al. "Developments in particle tracking using the Birmingham Positron Camera." Nuclear Instruments and Methods in Physics Research A 392 (1997b): 421-426.

Parker, D J, M R Hawkesworth, C J Broadbent, P Fowles, T D Fryer, and P A McNeil. "Industrial positron-based imaging: principles and applications." Nuclear Instruments and Methods in Physics Research A 348 (1994): 583-592.

Parker, D J, R N Forster, $\mathrm{P}$ Fowles, and $\mathrm{P} \mathrm{S}$ Takhar. "Positron emission particle trackingusingthe new Birmingham positron camera." Nuclear Instruments and Methods in Physics Research A 477 (2002): 540-545.

Radziszewski, P. "Comparing three DEM charge motion models." Minerals Engineering 12, no. 12 (1999): 1501-1520.

Radziszewski, P, Y Y Quan, and J Poirier. "Design Parameters Affecting Tumbling Mill Natural Frequencies." CDEN conference. Kananaskis, Alberta, 2005.

Rapaport, D C. "Mechanism for granular segregation." Physical Review E 64 (2001): 061304.

Rapaport, D C. "Radial and axial segregation of granular matter in a rotating cylinder: A simulation study." PHYSICAL REVIEW E 75 (2007): 031301.

Santomaso, A C, Y L Ding, J R Lickiss, and D W York. "Investigation of the Granular Behaviour in a Rotating Drum Operated over a Wide Range of Rotational Speed." Institution of Chemical Engineers 81 (2003): 936-945.

Shalizi, C. Almost None of the Theory of Stochastic Processes. 2007.

Songfack, P, and R Rajamani. "Hold-up studies in a pilot scale continuous ball mill: dynamic variations due to changes in operating variables." International Journal of Mineral Processing 57 (1999): 105-123. 
Sposito, Garrison. "Ergodicity and the 'scale effect'." Advances in Water Resources 20, no. 56 (1997): 309-316.

Standish, N, and A B Yu. "Porosity Calculations of Ternary Mixtures of particles." Powder Technology 49 (1987): 249-253.

Taberlet, N, P Richard, and E John Hinch. "S shape of a granular pile in a rotating drum." Physical Review E 73 (2006): 050301-1 - 050301-4.

US National Materials Advisory Board. Evolutionary and revolutionary technologies for mining. Technical Report, National Academic of Sciences, 2002.

von Neumann, Jon. "Proof of the Quasi-ergodic Hypothesis." Proceedings of the National Academy of Sciences of the United States of America. National Academy of Sciences, 1932. 70-82.

Walters, P. An introduction to ergodic theory. Springer-Verlag, 1982.

Wildman, R D, and D J Parker. "Coexistence of Two Granular Temperatures in Binary Vibrofluidized Beds." PHYSICAL REVIEW LETTERS 88, no. 6 (2002).

Wildman, R D, J M Huntley, J -P Hansen, D J Parker, and D A Allen. "Single-particle motion in three-dimensional vibrofluidized granular beds." Physical Review E 62, no. 3 (2000): 3826-3835.

Yang, R Y, A B Yu, L McElroy, and J Bao. "Numerical simulation of particle dynamics in different flowregimes in a rotating drum." Powder Technology, no. 188 (2008): 170-177.

Yang, R Y, R P Zou, and A B Yu. "Microdynamic analysis of particle flow in a horizontal rotating drum." Powder Technology, no. 130 (2003): 138-146.

$\mathrm{Yu}, \mathrm{A} \mathrm{B}$, and N Standish. "Estimation of the Porosity of Particle Mixtures by a LinearMixture Packing Model." Ind. Eng. Chem. Res. 30 (1991): 1372-1385.

Yu, A B, and N Standish. "Porosity Calculations of Multi-component Mixtures of Spherical Particles." Powder Technology 52 (1987): 233-241.

Zhou, Y C, A B Yu, R L Stewart, and J Bridgwater. "Microdynamic analysis of the particle flow in a cylindrical bladed mixer." Chemical Engineering Science 59 (2004): 1343-1364. 
